\title{
A Biomimetically Inspired, Efficient Synthesis of the South 7 Hemisphere of Cephalostatin $7 .^{\dagger}$
}

\author{
Jong Seok Lee and Philip L. Fuchs* \\ Department of Chemistry, 560 Oval Drive, Purdue University, West Lafayette, Indiana \\ 47907 \\ pfuchs@purdue.edu
}

\section{Supporting Information}


General. All reactions were carried out under nitrogen. Methylene chloride $\left(\mathrm{CH}_{2} \mathrm{Cl}_{2}\right)$ were dried and distilled from calcium hydride $\left(\mathrm{CaH}_{2}\right)$. All work-up, wash, recrystalization, and chromatographic solvents were distilled. Sodium sulfate $\left(\mathrm{Na}_{2} \mathrm{SO}_{4}\right)$ was anhydrous. DMSO was freshly distilled and powdered molecular sieves (4 $\AA$ ) were pre-activated by heating in vacuo at $300{ }^{\circ} \mathrm{C}$ for $15 \mathrm{~h}$. Aluminum oxide $\left(\mathrm{Al}_{2} \mathrm{O}_{3}\right)$, activated, neutral, Brockmann I was used. Thin layer chromatography (TLC) was used to monitor the progress of reactions by co-spotting with the starting materials. $p$-Anisaldehyde (1350 $\mathrm{mL}$ absolute ethanol, $50 \mathrm{~mL}$ concentrated $\mathrm{H}_{2} \mathrm{SO}_{4}, 37 \mathrm{~mL}$-anisaldehyde) was utilized as a common TLC visualizing solution.

Flash chromatographic purifications were performed using silica gel (230-400 mesh). ${ }^{1} \mathrm{H}$ NMR and ${ }^{13} \mathrm{C}$ NMR data were recorded on Varian INOVA-300 in chloroform- $\mathrm{d}_{1}$ as a solvent and are described in parts per million (ppm) from the residual chloroform (7.24ppm and 77.0ppm).

Peak multiplicates in ${ }^{1} \mathrm{H}$ NMR spectra are abbreviated as s (singlet), $t$ (triplet), $d d$ (doublet of doublet), dt (doublet of triplet), m (multiplet), br (broad), q (quartet), quintet and br s (broad singlet). Mass spectra were performed by the Purdue University campus wide mass spectrometry facility. 


\section{Characterization Check List}

\begin{tabular}{|c|c|c|c|c|c|}
\hline Compound & ${ }^{1} \mathrm{H}$ NMR & ${ }^{13} \mathrm{C}$ NMR & ${ }^{19} \mathrm{~F}$ NMR & HRMS & LRMS \\
\hline & $\mathrm{O}$ & $\mathrm{O}$ & $\mathrm{O}$ & ESI & ESI \\
\hline & $\mathrm{O}$ & $\mathrm{O}$ & NA & ESI & ESI \\
\hline & $\mathrm{O}$ & $\mathrm{O}$ & NA & ESI & ESI \\
\hline & $\mathrm{O}$ & $\mathrm{O}$ & NA & ESI & ESI \\
\hline & $\mathrm{O}$ & $\mathrm{O}$ & NA & ESI & ESI \\
\hline & $\mathrm{O}$ & $\mathrm{O}$ & NA & ESI & ESI \\
\hline $\mathrm{AcO}$ & & & & & \\
\hline
\end{tabular}




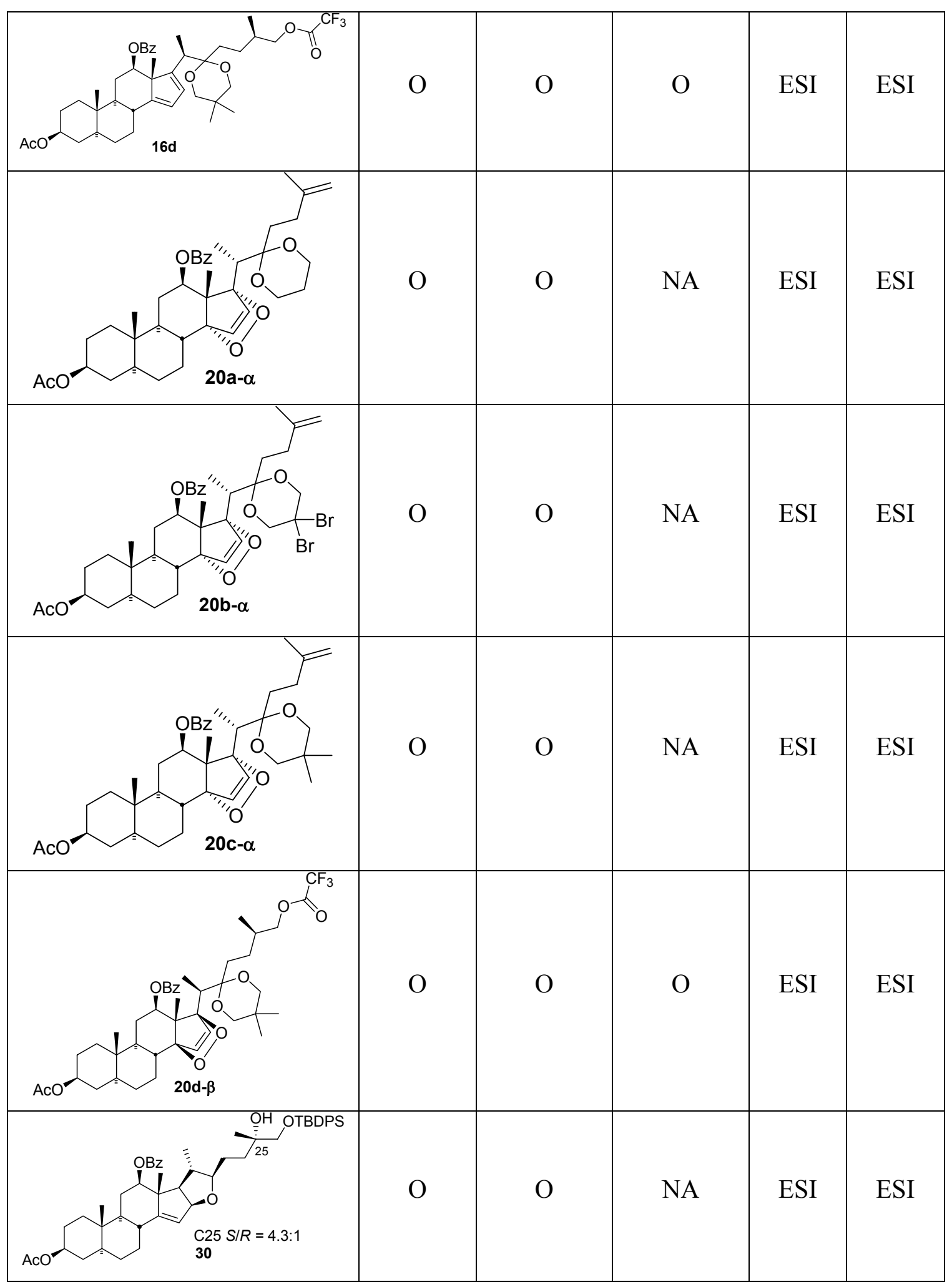




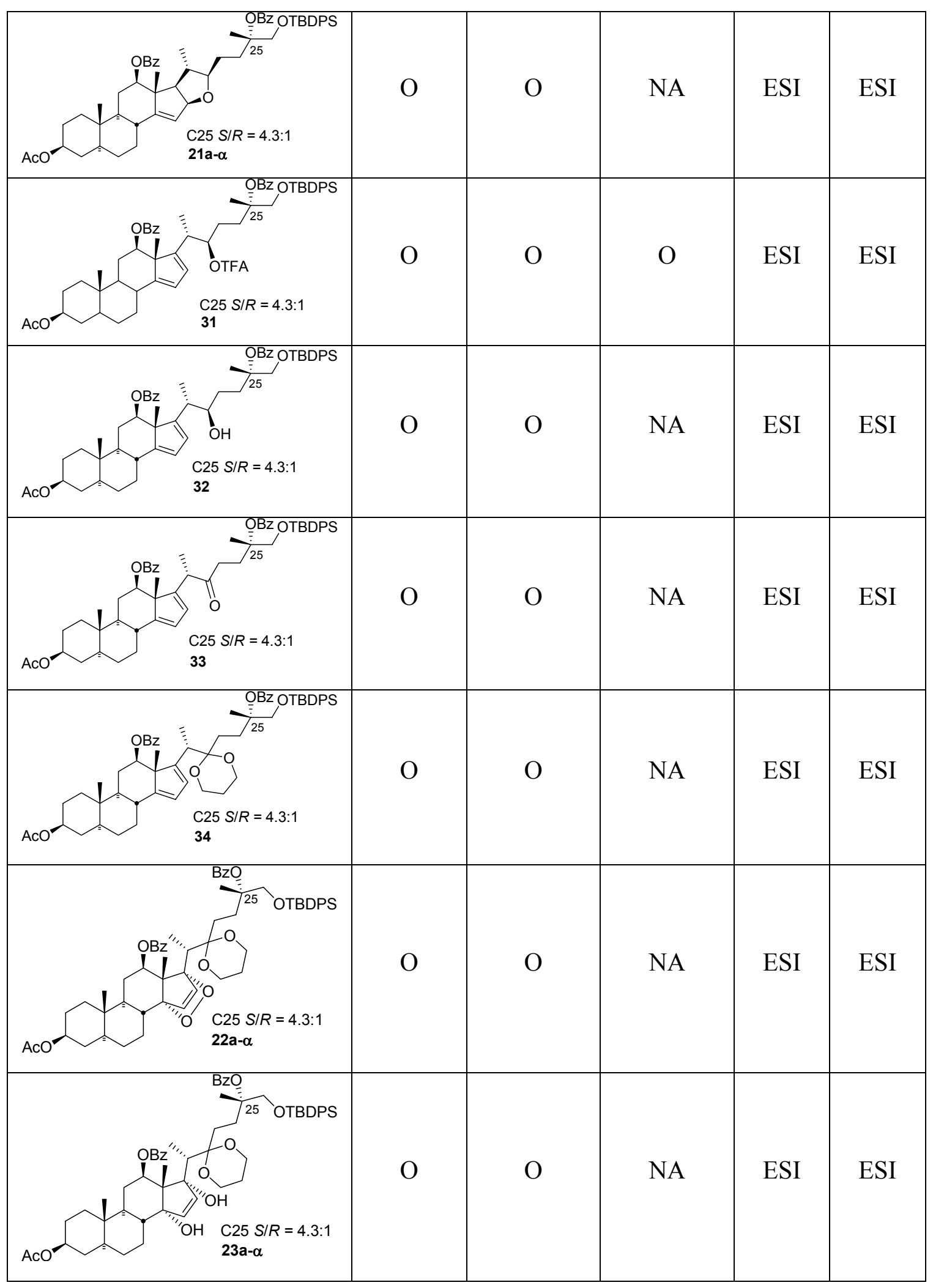




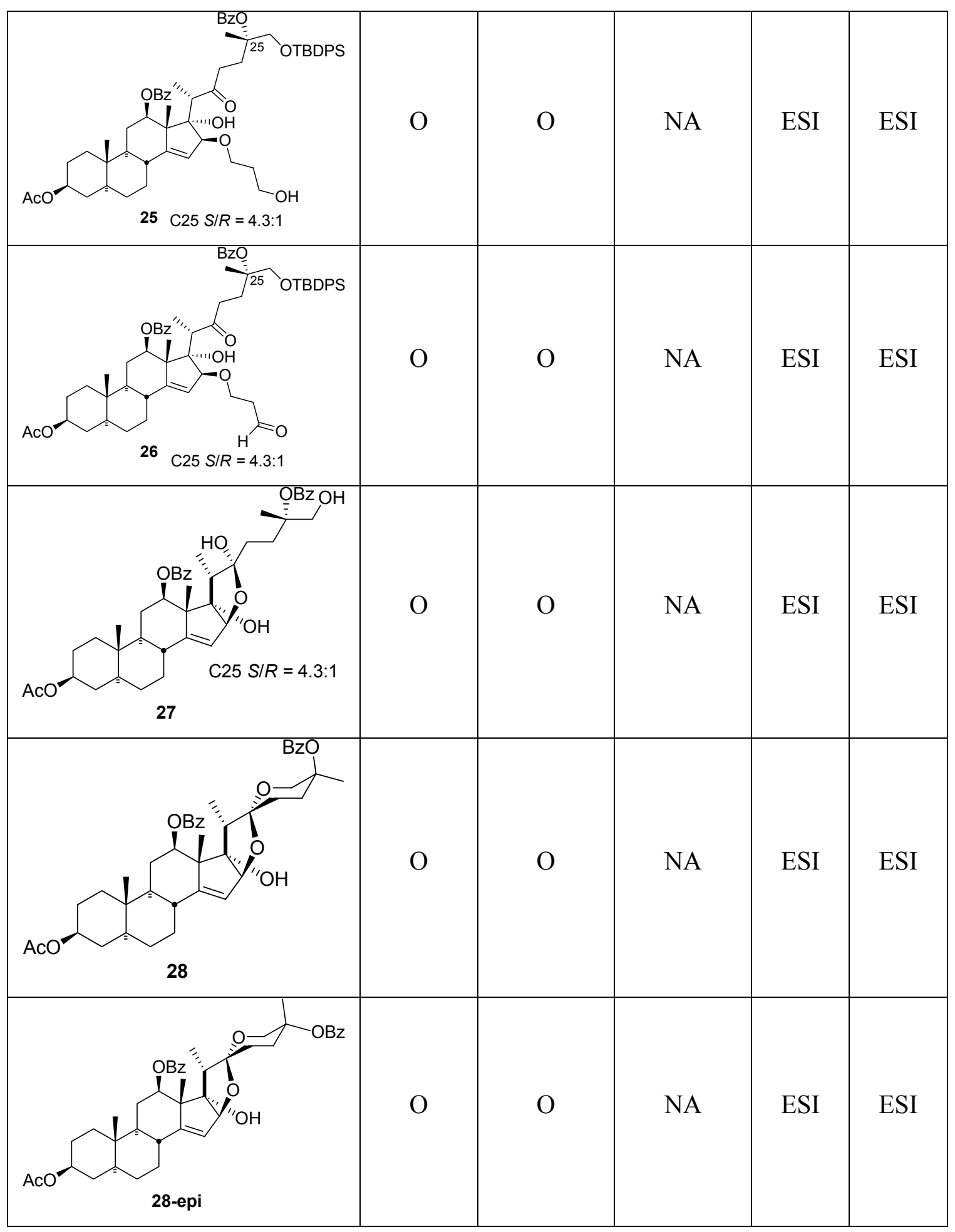




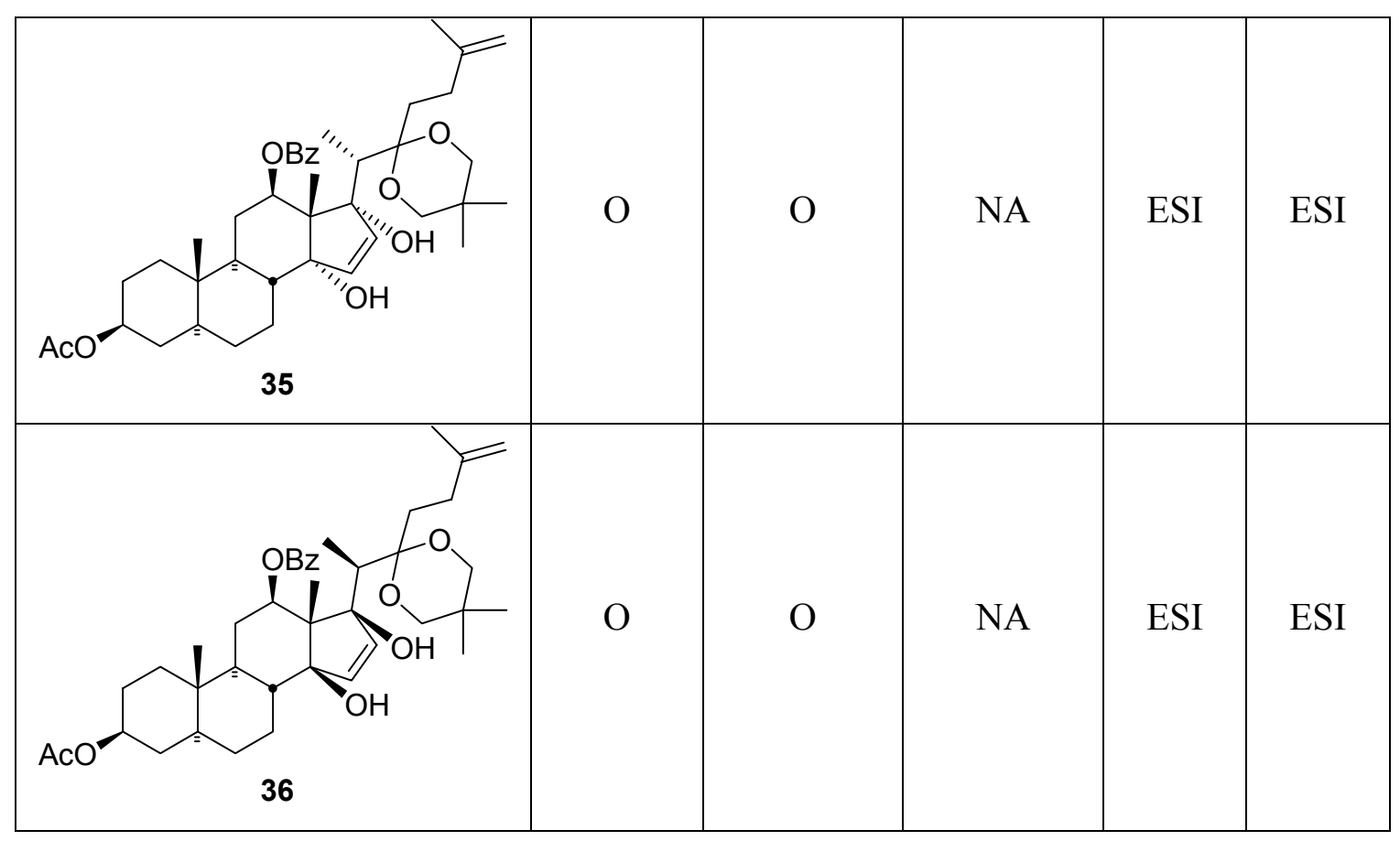




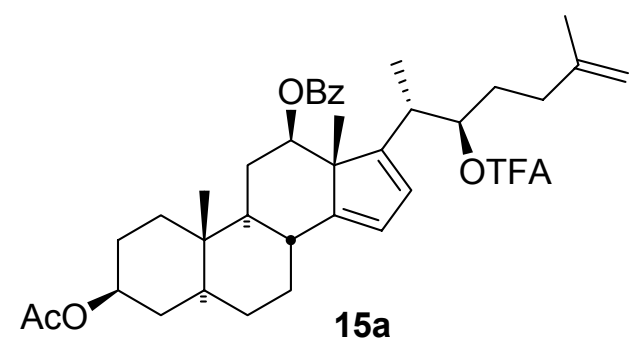

Dienyl trifluoroacetate 15a. To a solution of terminal olefin 14 (258.8 mg, 0.4615 mmol) and 2,6-di-tert-butyl-4-methyl pyridine (113.7 mg, $0.5538 \mathrm{mmol}, 1.2$ equiv) in $\mathrm{CH}_{2} \mathrm{Cl}_{2}(3 \mathrm{~mL})$ was added freshly prepared TFAT (105.5 $\mu \mathrm{L}, 0.6923 \mathrm{mmol}, 1.5$ equiv) at $-30{ }^{\circ} \mathrm{C}$. The reaction was stirred for $1 \mathrm{~h}$ at $-30 \sim-40{ }^{\circ} \mathrm{C}$. The reaction was quenched by adding sat. $\mathrm{NaHCO}_{3}$ and diluted with EtOAc. The organic layer was washed with brine, dried over $\mathrm{Na}_{2} \mathrm{SO}_{4}$, filtered, and concentrated in vacuo. The crude residue was purified by flash column chromatography (EtOAc/n-Hexane $=1: 4)$ to give dienyl trifluoroacetate 7 (294.0 mg, $0.4476 \mathrm{mmol}, 97 \%$ ) as white solid; mp $118 \sim 119{ }^{\circ} \mathrm{C}\left(\mathrm{CH}_{2} \mathrm{Cl}_{2}\right) ;{ }^{1} \mathrm{H}$ NMR (300 $\left.\mathrm{MHz}, \mathrm{CDCl}_{3}\right) \delta 8.03(\mathrm{~d}, J=7.5 \mathrm{~Hz}, 2 \mathrm{H}), 7.53-7.58(\mathrm{~m}, 1 \mathrm{H}), 7.41-7.46(\mathrm{~m}, 2 \mathrm{H}), 6.16$ (d, $J=1.6 \mathrm{~Hz}, 1 \mathrm{H}), 5.9(\mathrm{~s}, 1 \mathrm{H}), 5.04-5.10(\mathrm{~m}, 1 \mathrm{H}), 4.63-4.71(\mathrm{~m}, 2 \mathrm{H}), 4.56(\mathrm{~s}, 1 \mathrm{H})$, 4.39 (dd, $J=11.3,4.2 \mathrm{~Hz}, 1 \mathrm{H}), 2.89$ (dq, $J=6.9,6.8 \mathrm{~Hz}, 1 \mathrm{H}), 2.19(\mathrm{br} \mathrm{s}, 11.0 \mathrm{~Hz}, 1 \mathrm{H})$, 1.98 (s, 3H), 1.61 (s, 3H), 1.26 (s, 3H), 0.90 (s, 3H), 0.86 (d, $J=7.0 \mathrm{~Hz}, 3 \mathrm{H}) ;{ }^{13} \mathrm{C}$ NMR $\left(75 \mathrm{MHz}, \mathrm{CDCl}_{3}\right) \delta 170.4,165.4,156.4,155.8,143.9,133.0,130.2,129.3,128.4,126.4$, $120.8,110.8,81.8,79.3,73.2,56.9,53.1,44.2,36.9,35.7,35.6,34.6,33.7,32.8,29.0$, 28.0, 27.8, 27.3, 27.1, 22.1, 21.2, 17.8, 13.4, 12.1; ${ }^{19} \mathrm{~F}$ NMR (282 MHz, $\left.\mathrm{CDCl}_{3}\right) \delta$-75.06; HRMS (ESI) $\mathrm{C}_{38} \mathrm{H}_{47} \mathrm{~F}_{3} \mathrm{O}_{6} \mathrm{Na}[\mathrm{M}+\mathrm{Na}]^{+}$calcd. 679.3223, found 679.3221 .

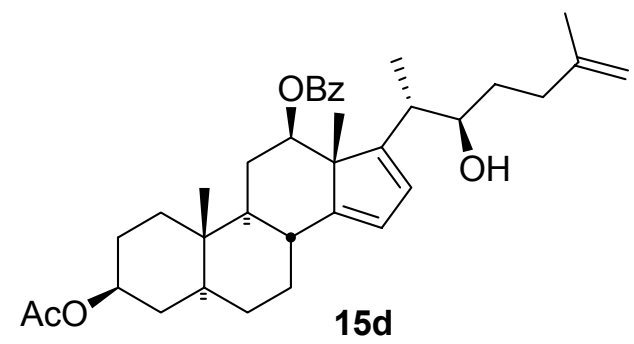

Secondary alcohol 15d. Dienyl trifluoroacetate 15a (294 mg, $0.4476 \mathrm{mmol})$ was stirred with $\mathrm{Na}_{2} \mathrm{CO}_{3}$ (189.8 mg, $1.7906 \mathrm{mmol}, 4$ equiv) in 8:1:1 mixture of THF, water, and $\mathrm{MeOH}$ at room temperature for $7 \mathrm{~h}$. The reaction was extracted with EtOAc three times. 
The extract was washed with brine, dried over $\mathrm{Na}_{2} \mathrm{SO}_{4}$, filtered, and concentrated in vacuo. The crude residue was purified by flash column chromatography (EtOAc/nHexane $=1: 8)$ to give secondary alcohol $\mathbf{1 5 d}(242.1 \mathrm{mg}, 0.4317 \mathrm{mmol}, 96 \%)$ as white solid; mp $76 \sim 77{ }^{\circ} \mathrm{C}\left(\mathrm{CH}_{2} \mathrm{Cl}_{2} / \mathrm{n}\right.$-Hexane); ${ }^{1} \mathrm{H}$ NMR $\left(300 \mathrm{MHz}, \mathrm{CDCl}_{3}\right) \delta 8.06(\mathrm{~d}, J=7.18$ $\mathrm{Hz}, 2 \mathrm{H}), 7.55-7.59$ (m, 1H), $7.42-7.47$ (m, 2H), 6.18 (d, $J=1.8 \mathrm{~Hz}, 1 \mathrm{H}), 5.95$ (s, 1H), $4.62-4.73(\mathrm{~m}, 3 \mathrm{H}), 4.44(\mathrm{~d}, J=11.2,4.2 \mathrm{~Hz}, 1 \mathrm{H}), 3.54(\mathrm{dt}, J=8.6,2.2 \mathrm{~Hz}, 1 \mathrm{H}), 2.56$ (dq, $J=7.6,6.9 \mathrm{~Hz}, 1 \mathrm{H}), 2.03-2.24(\mathrm{~m}, 2 \mathrm{H}), 2.00(\mathrm{~s}, 3 \mathrm{H}), 1.67$ (s, 3H), $1.26(\mathrm{~s}, 3 \mathrm{H})$, $0.91(\mathrm{~s}, 3 \mathrm{H}), 0.78(\mathrm{~d}, J=6.7 \mathrm{~Hz}, 3 \mathrm{H}) ;{ }^{13} \mathrm{C} \mathrm{NMR}\left(75 \mathrm{MHz}, \mathrm{CDCl}_{3}\right) \delta 170.3,165.4,160.0$, 155.8, 145.7, 132.9, 130.4, 129.2, 128.3, 124.7, 120.6, 109.6, 79.0, 75.1, 73.1, 56.9, 53.0, 44.1, 39.0, 36.8, 35.6, 34.6, 33.6, 33.4, 31.7, 29.0, 28.0, 27.2, 27.1, 22.4, 21.2, 19.5, 14.1, 12.0; HRMS (ESI) $\mathrm{C}_{36} \mathrm{H}_{48} \mathrm{O}_{5} \mathrm{Na}[\mathrm{M}+\mathrm{Na}]^{+}$calcd. 583.3399, found 583.3402.

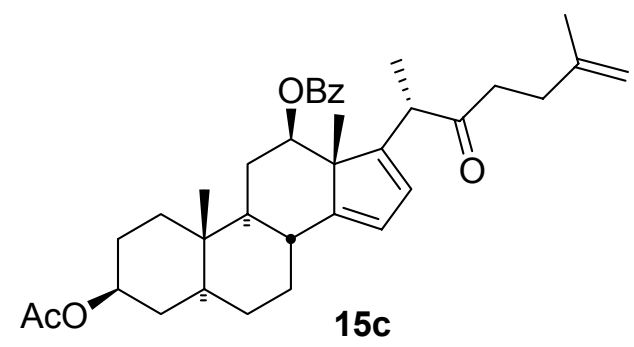

Dienyl ketone 15c. To a solution of freshly distilled DMSO $(0.6 \mathrm{~mL}, 8.02 \mathrm{mmol}, 3$ equiv) in $\mathrm{CH}_{2} \mathrm{Cl}_{2}(2 \mathrm{~mL})$ was added TFAA $\left(0.94 \mathrm{~mL}, 6.69 \mathrm{mmol}, 2.5\right.$ equiv) at $-78{ }^{\circ} \mathrm{C}$. The solution was stirred for $20 \mathrm{~min}$. at $-78{ }^{\circ} \mathrm{C}$. To the solution, secondary alcohol $\mathbf{1 5 d}$ (1.50 g, $2.67 \mathrm{mmol})$ in $\mathrm{CH}_{2} \mathrm{Cl}_{2}(25 \mathrm{~mL})$ was added dropwise using a dropping funnel for $20 \mathrm{~min}$. at $-78{ }^{\circ} \mathrm{C}$. The reaction was stirred for $40 \mathrm{~min}$. at $-78{ }^{\circ} \mathrm{C}$, and then allowed to warm to room temperature. The reaction was stirred for additional $6 \mathrm{~h}$ at room temperature. After $6 \mathrm{~h}$, the reaction was diluted with EtOAc, worked up with water, washed with brine, and dried over $\mathrm{Na}_{2} \mathrm{SO}_{4}$. After removal of solvent, the crude residue was purified by flash column chromatography $(\mathrm{EtOAc} / \mathrm{n}-\mathrm{Hexane}=1: 8)$ to provide dienyl ketone 15c (1.41 g, $2.5 \mathrm{mmol}, 94 \%$ ) as white solid; mp $65 \sim 66{ }^{\circ} \mathrm{C}$ (EtOAc/n-Hexane); ${ }^{1} \mathrm{H}$ NMR (300 MHz, $\left.\mathrm{CDCl}_{3}\right) \delta 8.07(\mathrm{~d}, J=7.5 \mathrm{~Hz}, 2 \mathrm{H}), 7.54-7.59(\mathrm{~m}, 1 \mathrm{H}), 7.43-7.48(\mathrm{~m}$, 2H), $6.09(\mathrm{~d}, J=1.6 \mathrm{~Hz}, 1 \mathrm{H}), 5.92(\mathrm{~s}, 1 \mathrm{H}), 4.63-4.71(\mathrm{~m}, 2 \mathrm{H}), 4.55$ (s, 1H), 4.42 (dd, $J$ $=6.9 \mathrm{~Hz}, 1 \mathrm{H}), 3.48(\mathrm{q}, J=7.0 \mathrm{~Hz}, 1 \mathrm{H}), 2.37-2.61(\mathrm{~m}, 2 \mathrm{H}), 2.15-2.24(\mathrm{~m}, 3 \mathrm{H}), 1.99(\mathrm{~s}$, $3 \mathrm{H}), 1.65(\mathrm{~s}, 3 \mathrm{H}), 1.28(\mathrm{~s}, 3 \mathrm{H}), 1.02(\mathrm{~d}, J=6.7 \mathrm{~Hz}, 3 \mathrm{H}), 0.9(\mathrm{~s}, 3 \mathrm{H}) ;{ }^{13} \mathrm{C} \mathrm{NMR}(75 \mathrm{MHz}$, 
$\left.\mathrm{CDCl}_{3}\right) \delta 209.4,170.3,165.3,156.4,154.4,144.4,133.0,130.2,129.3,128.4,127.4$, 121.0, 109.9, 78.8, 73.1, 56.8, 52.8, 45.9, 44.1, 38.3, 36.8, 35.7, 34.7, 33.6, 31.7, 29.0, 27.9, 27.2, 27.1, 22.5, 21.2, 17.8, 14.2, 12.0; HRMS (ESI) $\mathrm{C}_{36} \mathrm{H}_{46} \mathrm{O}_{5} \mathrm{Na}[\mathrm{M}+\mathrm{Na}]^{+}$calcd. 581.3243 , found 581.3247 .

\section{General procedure for cyclic ketalization of ketone $15 \mathrm{c}{ }^{1}$}

To a solution of ketone $15 \mathrm{c}$ and $10 \mathrm{~mol} \%$ of $\mathrm{Sc}(\mathrm{OTf})_{3}$ in $\mathrm{CH}_{3} \mathrm{CN}(0.1 \mathrm{M})$ was added 10 eq. 1,3-propanediol, followed by 10 eq. $\mathrm{HC}(\mathrm{OMe})_{3}$. The reaction was stirred at room temperature until the ketone 15c completely disappeared on TLC. The reaction was diluted with EtOAc, worked up with water, washed with brine, and dried over $\mathrm{Na}_{2} \mathrm{SO}_{4}$. After removal of solvent, the crude residue was treated with 10 eq. $\mathrm{Ac}_{2} \mathrm{O}$ and $10 \mathrm{~mol} \%$ DMAP in pyridine at room temperature for $4 \mathrm{~h}$. As the reaction was completed, pyridine was removed by $10 \% \mathrm{HCl}$ and EtOAc. The organic layer was neutralized by sat. $\mathrm{NaHCO}_{3}$, washed with brine, dried over $\mathrm{Na}_{2} \mathrm{SO}_{4}$ and evaporated in vacuo. The residue was purified by flash column chromatography to provide corresponding cyclic ketal.

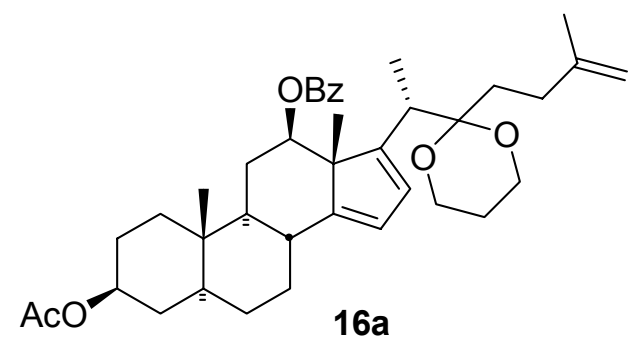

Diene 16a. Prepared in $91 \%$ yield following the above general procedure; white solid; mp 88 89 ${ }^{\circ} \mathrm{C}\left(\mathrm{CH}_{2} \mathrm{Cl}_{2} / \mathrm{n}-\mathrm{Hexane}\right) ;{ }^{1} \mathrm{H}$ NMR $\left(300 \mathrm{MHz}, \mathrm{CDCl}_{3}\right) \delta 8.02-8.07(\mathrm{~m}, 2 \mathrm{H})$, $7.39-7.45$ (m, 1H), $7.49-7.56$ (m, 2H), 6.37 (d, $J=1.2 \mathrm{~Hz}, 1 \mathrm{H}), 5.93$ (s, 1H), $4.66-$ $4.71(\mathrm{~m}, 1 \mathrm{H}), 4.62(\mathrm{~s}, 1 \mathrm{H}), 4.54(\mathrm{~s}, 1 \mathrm{H}), 3.77-3.92(\mathrm{~m}, 3 \mathrm{H}), 3.61-3.65(\mathrm{~m}, 1 \mathrm{H}), 2.83(\mathrm{q}$, $J=7.03 \mathrm{~Hz}, 1 \mathrm{H}), 1.99$ (s, 3H), 1.61 (s, 3H), 1.22 (s, 3H), 0.90 (s, 3H), 0.84 (d, $J=7.0 \mathrm{~Hz}$, $3 \mathrm{H}) ;{ }^{13} \mathrm{C} \mathrm{NMR}\left(75 \mathrm{MHz}, \mathrm{CDCl}_{3}\right) \delta 170.6,165.5,158.2,154.2,145.9,132.9,130.7,129.4$,

\footnotetext{
${ }^{1}$ (a) Ishihara, K.; Karumi, Y.; Kubota, M.; Yamamoto, H. Synlett. 1996, 839. (b) Fukuzawa, S.;

Tsuchimoto, T.; Hotaka, T.; Hiyama, T. Synlett. 1995, 1077.
} 
$128.4,126.7,121.6,109.4,101.5,79.9,73.4,59.4,59.1,57.2,53.3,44.3,37.7,37.0,35.8$, $34.7,33.8,31.6,29.2,28.2,27.5,27.2,26.2,25.2$, 22.7, 21.4, 17.5, 13.9, 12.2; HRMS (ESI) $\mathrm{C}_{39} \mathrm{H}_{52} \mathrm{O}_{6} \mathrm{Na}[\mathrm{M}+\mathrm{Na}]^{+}$calcd. 639.3662, found 639.3661 .

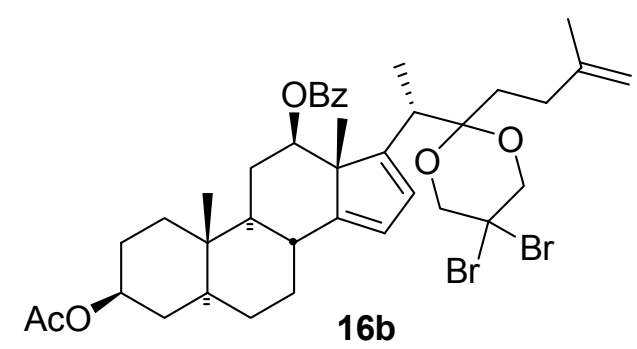

Diene 16b. ${ }^{2}$ Prepared in 53\% yield following the above general procedure; oil; ${ }^{1} \mathrm{H}$ NMR $\left(300 \mathrm{MHz}, \mathrm{CDCl}_{3}\right) \delta 8.06(\mathrm{~d}, J=7.6 \mathrm{~Hz}, 2 \mathrm{H}), 7.52-7.57(\mathrm{~m}, 1 \mathrm{H}), 7.41-7.46(\mathrm{~m}, 2 \mathrm{H})$, $6.41(\mathrm{~d}, J=1.8 \mathrm{~Hz}, 1 \mathrm{H}), 5.92(\mathrm{~s}, 1 \mathrm{H}), 4.65-4.71(\mathrm{~m}, 2 \mathrm{H}), 4.55(\mathrm{~s}, 1 \mathrm{H}), 4.42$ (dd, $J=$ $11.1,4.7 \mathrm{~Hz}, 1 \mathrm{H}), 4.06-4.20(\mathrm{~m}, 3 \mathrm{H}), 3.96-4.00(\mathrm{~d}, J=12.9 \mathrm{~Hz}, 1 \mathrm{H}), 2.93(\mathrm{q}, J=7.0$ $\mathrm{Hz}, 1 \mathrm{H}), 2.15-2.22$ (m, 2H), 2.00 (s, 3H), 1.61 (s, 3H), 1.23 (s, 3H), 0.94 (d, $J=7.0 \mathrm{~Hz}$, $3 \mathrm{H}), 0.91(\mathrm{~s}, 3 \mathrm{H}) ;{ }^{13} \mathrm{C} \mathrm{NMR}\left(75 \mathrm{MHz}, \mathrm{CDCl}_{3}\right) \delta 170.6,165.4,155.8,154.7,145.0,133.0$, 130.6, 129.4, 128.5, 121.5, 109.9, 103.0, 80.0, 73.4, 72.6, 72.4, 59.7, 57.1, 53.3, 44.3, $37.3,37.0,35.8,34.6,33.8,31.6,31.3,29.2,28.2,27.5,27.2,25.8,25.3,22.7,22.6,21.4$, 17.8, 14.1, 13.7, 12.2; HRMS (ESI) $\mathrm{C}_{39} \mathrm{H}_{50} \mathrm{Br}_{2} \mathrm{O}_{6} \mathrm{Na}[\mathrm{M}+\mathrm{Na}]^{+}$calcd. 795.1872, found 795.1880 .

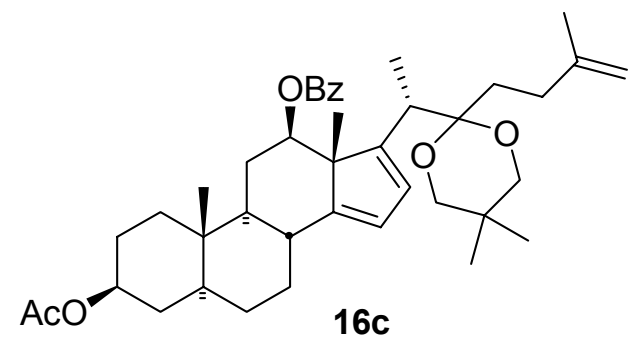

Diene 16c. Prepared in 91\% yield following the above general procedure; mp 214 215 ${ }^{\circ} \mathrm{C}\left(\mathrm{CH}_{2} \mathrm{Cl}_{2} / \mathrm{n}\right.$-Hexane); ${ }^{1} \mathrm{H}$ NMR $\left(300 \mathrm{MHz}, \mathrm{CDCl}_{3}\right) \delta 8.06(\mathrm{~d}, J=7.2 \mathrm{~Hz}, 2 \mathrm{H}), 7.50-$ 7.55 (m, 1H), $7.39-7.44$ (m, 2H), 6.37 (d, $J=1.8 \mathrm{~Hz}, 1 \mathrm{H}), 5.92$ (s, 1H), $4.63-4.71$ (m,

\footnotetext{
${ }^{2}$ For preparation of 2,2-Dibromo-1,3-propanediol see Corey, E. J.; Trybulski, E. J.; Suggs, J. W. Tetrahedron Lett. 1976, 17, 4577.
} 
1H), 4.60 (s, 1H), 4.52 (s, 1H), 3.52 (dd, $J=20.4,11.3 \mathrm{~Hz}, 2 \mathrm{H}), 3.31$ (d, $J=10.1 \mathrm{~Hz}, 1 \mathrm{H})$, $3.17(\mathrm{~d}, J=11.0 \mathrm{~Hz}, 1 \mathrm{H}), 2.88(\mathrm{q}, J=7.0 \mathrm{~Hz}, 1 \mathrm{H}), 2.13-2.22(\mathrm{~m}, 1 \mathrm{H}), 1.99(\mathrm{~s}, 3 \mathrm{H})$, 1.59 (s, 3H), 1.22 (s, 3H), 0.94 (s, 3H), 0.90 (s, 3H), 0.87 (d, $J=7.5 \mathrm{~Hz}, 3 \mathrm{H}), 0.68$ (s,

$3 \mathrm{H}) ;{ }^{13} \mathrm{C}$ NMR $\left(75 \mathrm{MHz}, \mathrm{CDCl}_{3}\right) \delta 170.5,165.5,157.8,154.3,145.8,132.9,130.6,129.4$, $128.4,127.6,121.4,109.3,101.4,80.0,73.4,69.9,69.6,57.1,53.3,44.3,37.4,36.9,35.8$, $34.6,33.8,31.5,29.5,29.2,28.2,27.5,27.2,25.3,23.1,22.7,22.6,22.5,21.4,17.7,14.1$, 13.6, 12.2; HRMS (ESI) $\mathrm{C}_{41} \mathrm{H}_{56} \mathrm{O}_{6} \mathrm{Na}[\mathrm{M}+\mathrm{Na}]^{+}$calcd. 667.3975, found 667.3980.

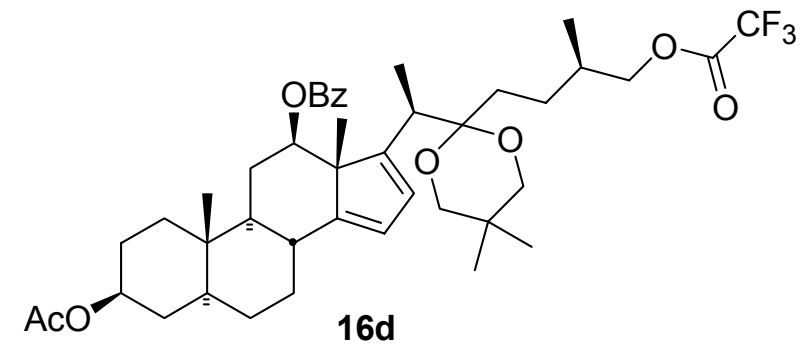

Diene 16d. ${ }^{3}$ To a solution of dienyl ketone $13(474.9 \mathrm{mg}, 0.7059 \mathrm{mmol}, 1: 8$ mixture of $\mathrm{C} 21-\alpha-\mathrm{Me} / \mathrm{C} 21-\beta-\mathrm{Me})$ and $\mathrm{TMSOCH}_{2} \mathrm{C}\left(\mathrm{CH}_{3}\right)_{2} \mathrm{CH}_{2} \mathrm{OTMS}(0.7 \mathrm{~mL}, 2.8235 \mathrm{mmol}, 4$ eq. $)$ in $\mathrm{CH}_{2} \mathrm{Cl}_{2}(5 \mathrm{~mL}, 0.14 \mathrm{M})$ was added TMSOTf $(12.8 \mu \mathrm{L}, 10 \mathrm{~mol} \%)$ at $-20{ }^{\circ} \mathrm{C}$. The reaction was allowed to warm to room temperature. After $9.5 \mathrm{~h}$, the reaction was quenched by adding $0.1 \mathrm{~mL}$ pyridine, and extracted with water/ethyl acetate, washed with brine, and dried over $\mathrm{Na}_{2} \mathrm{SO}_{4}$. After removal of solvent, the crude residue was purified by flash column chromatography ( $\mathrm{n}-\mathrm{Hexane}$ only to EtOAc/n-Hexane $=1: 8$ ) to provide diene 16d (425.3 mg, $0.5604 \mathrm{mmol}, 1: 7$ mixture of $\mathrm{C} 21-\alpha-\mathrm{Me} / \mathrm{C} 21-\beta-\mathrm{Me}, 79 \%$ ); oil; ${ }^{1} \mathrm{H}$ NMR (300 MHz, $\left.\mathrm{CDCl}_{3}\right) \delta 8.06(\mathrm{~d}, J=7.3 \mathrm{~Hz}, 2 \mathrm{H}), 7.53-7.58(\mathrm{~m}, 1 \mathrm{H}), 7.40-7.45(\mathrm{~m}$, 2H), 6.34 (d, $J=1.8 \mathrm{~Hz}, 1 \mathrm{H}), 5.91(\mathrm{~s}, 1 \mathrm{H}), 4.63-4.71(\mathrm{~m}, 1 \mathrm{H}), 4.45$ (dd, $J=11.3,4.4$ $\mathrm{Hz}, 1 \mathrm{H}), 4.16$ (ddd, $J=14.2,6.7,5.0 \mathrm{~Hz}, 2 \mathrm{H}), 3.31-3.52(\mathrm{~m}, 3 \mathrm{H}), 3.15(\mathrm{~d}, J=11.1 \mathrm{~Hz}$, $1 \mathrm{H}), 2.14-2.22(\mathrm{~m}, 1 \mathrm{H}), 1.99$ (s, 3H), 1.20 (s, 3H), 0.91 (s, 3H), 0.86 (s, 6H), 0.77 (d, $J$ $=6.6 \mathrm{~Hz}, 3 \mathrm{H}), 0.69(\mathrm{~s}, 3 \mathrm{H}) ;{ }^{13} \mathrm{C} \mathrm{NMR}\left(75 \mathrm{MHz}, \mathrm{CDCl}_{3}\right) \delta 170.6,165.4,157.6,154.5$, 133.0, 130.7, 129.5, 128.4, 127.8, 121.4, 101.3, 79.7, 73.4, 72.6, 72.2, 72.1, 70.0, 69.6, $57.1,53.3,44.3,37.1,37.0,35.8,34.6,33.8,32.6,29.5,29.2,28.2,27.6,27.2,26.5,24.5$,

\footnotetext{
${ }^{3}$ Chiu, C. K.-F.; Mander, L. N.; Stuart, A. D.; Willis, A. C. Aust. J. Chem. 1992, 45, 227.
} 
23.0, 22.4, 21.4, 21.3, 17.8, 16.2, 13.5, 12.2, $-0.8 ;{ }^{19} \mathrm{~F}$ NMR (282 MHz, $\left.\mathrm{CDCl}_{3}\right) \delta$-75.38; HRMS (ESI) $\mathrm{C}_{43} \mathrm{H}_{57} \mathrm{~F}_{3} \mathrm{O}_{8} \mathrm{Na}[\mathrm{M}+\mathrm{Na}]^{+}$calcd. 781.3903, found 781.3900.

\section{General procedure for $[4+2]$ cycloaddition of dienes with singlet oxygen}

A solution of diene (16a-d) and 5,10,15,20-tetraphenyl-21H,23H-porphine, $0.5 \mathrm{~mol} \%$ TPP in $\mathrm{CH}_{2} \mathrm{Cl}_{2}(0.1 \mathrm{M})$ was purged with oxygen at $-78{ }^{\circ} \mathrm{C}$ for $5 \mathrm{~min}$. The reaction was stirred with irradiation by a sun lamp $\left(275 \mathrm{~W}\right.$ RS-M) at $-78^{\circ} \mathrm{C}$ for $40 \mathrm{~min}$. The reaction mixture was evaporated under reduced pressure at room temperature. TPP was removed by a short deactivated silica gel pad $(5 \times 5 \mathrm{~cm}$, EtOAc/n-Hexane $=5: 95)$ to give endoperoxide.

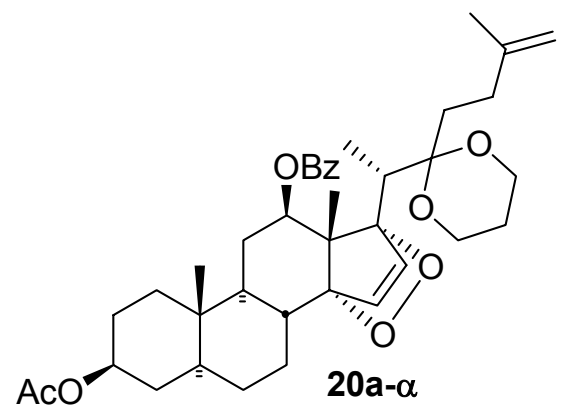

Endoperoxide 20a- $\alpha$. Prepared in $98 \%$ yield following the above general procedure; white solid; mp $140 \sim 141{ }^{\circ} \mathrm{C}\left(\mathrm{CH}_{2} \mathrm{Cl}_{2} / \mathrm{n}\right.$-Hexane); ${ }^{1} \mathrm{H}$ NMR (300 MHz, $\left.\mathrm{CDCl}_{3}\right) \delta 8.00$ (d, $7.6 \mathrm{~Hz}, 2 \mathrm{H}), 7.50-7.55(\mathrm{~m}, 1 \mathrm{H}), 7.37-7.42(\mathrm{~m}, 2 \mathrm{H}), 7.01(\mathrm{~d}, J=5.9 \mathrm{~Hz}, 1 \mathrm{H}), 6.28(\mathrm{~d}, J$ $=5.9 \mathrm{~Hz}, 1 \mathrm{H}), 5.57(\mathrm{dd}, J=11.1,4.7 \mathrm{~Hz}, 1 \mathrm{H}), 4.64-4.70(\mathrm{~m}, 1 \mathrm{H}), 4.55(\mathrm{~s}, 1 \mathrm{H}), 4.45(\mathrm{~s}$, $1 \mathrm{H}), 3.85-3.99(\mathrm{~m}, 2 \mathrm{H}), 3.67-3.80(\mathrm{~m}, 2 \mathrm{H}), 2.75$ (q, $J=7.0 \mathrm{~Hz}, 1 \mathrm{H}), 2.09-2.24(\mathrm{~m}$, $2 \mathrm{H}), 1.99$ (s, 3H), 1.51 (s, 3H), 1.19 (s, 3H), 0.89 (d, J=7.0 Hz, 3H), $0.82(\mathrm{~s}, 3 \mathrm{H}) ;{ }^{13} \mathrm{C}$ NMR (75 MHz, $\left.\mathrm{CDCl}_{3}\right) \delta 170.5,165.3,145.6,138.3,132.8,130.7,129.4,128.3,109.4$, 101.6, 97.2, 97.1, 73.3, 72.3, 64.6, 58.7, 45.2, 43.8, 36.5, 35.5, 34.9, 33.8, 33.7, 30.6, 28.5, 28.0, 27.2, 26.6, 24.4, 22.6, 21.4, 14.0, 11.5, 10.1; HRMS (ESI) $\mathrm{C}_{39} \mathrm{H}_{52} \mathrm{O}_{8} \mathrm{Na}[\mathrm{M}+\mathrm{Na}]^{+}$ calcd. 671.3560 , found 671.3555 . 


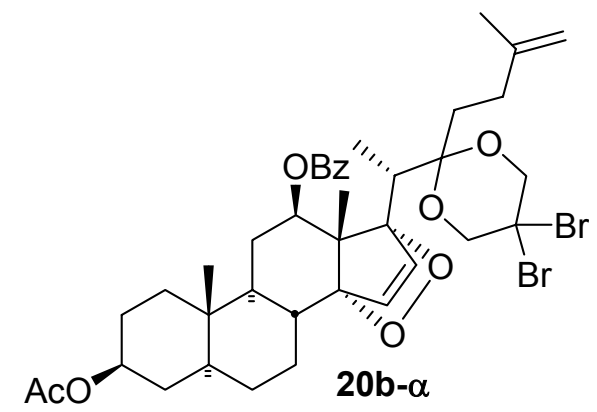

Endoperoxide 20b- $\alpha$. Prepared in $80 \%$ yield following the above general procedure; oil; ${ }^{1} \mathrm{H}$ NMR $\left(300 \mathrm{MHz}, \mathrm{CDCl}_{3}\right) \delta 7.98(\mathrm{~d}, J=7.6 \mathrm{~Hz}, 2 \mathrm{H}), 7.55-7.59$ (m, 1H), $7.42-7.47$ $(\mathrm{m}, 2 \mathrm{H}), 6.81(\mathrm{~d}, J=5.9 \mathrm{~Hz}, 1 \mathrm{H}), 6.36(\mathrm{~d}, J=5.9 \mathrm{~Hz}, 1 \mathrm{H}), 5.47(\mathrm{dd}, J=11.1,4.7 \mathrm{~Hz}$, $1 \mathrm{H}), 4.66-4.70(\mathrm{~m}, 1 \mathrm{H}), 4.63(\mathrm{~s}, 1 \mathrm{H}), 4.60(\mathrm{~s}, 1 \mathrm{H}), 3.96(\mathrm{dd}, J=18.7,12.9 \mathrm{~Hz}, 2 \mathrm{H})$, $3.20(\mathrm{q}, J=7.0 \mathrm{~Hz}, 1 \mathrm{H}), 2.02-2.27(\mathrm{~m}, 3 \mathrm{H}), 1.99(\mathrm{~s}, 3 \mathrm{H}), 1.64(\mathrm{~s}, 3 \mathrm{H}), 1.20(\mathrm{~s}, 3 \mathrm{H})$, $0.84(\mathrm{~d}, J=7.0 \mathrm{~Hz}, 3 \mathrm{H}), 0.81(\mathrm{~s}, 3 \mathrm{H}) ;{ }^{13} \mathrm{C} \mathrm{NMR}\left(75 \mathrm{MHz}, \mathrm{CDCl}_{3}\right) \delta 170.5,165.2,145.5$, $137.8,133.2$, 132.6, 130.5, 129.2, 128.6, 109.8, 102.7, 96.7, 95.7, 73.2, 72.7, 72.4, 72.2, 65.2, 58.6, 45.0, 43.7, 36.5, 35.5, 33.9, 33.7, 31.9, 31.0, 29.7, 28.4, 27.2, 27.1, 26.4, 22.7, 21.4, 14.3, 11.5, 9.4; HRMS (ESI) $\mathrm{C}_{39} \mathrm{H}_{50} \mathrm{Br}_{2} \mathrm{O}_{8} \mathrm{Na}[\mathrm{M}+\mathrm{Na}]^{+}$calcd. 827.1770, found 827.1781 .

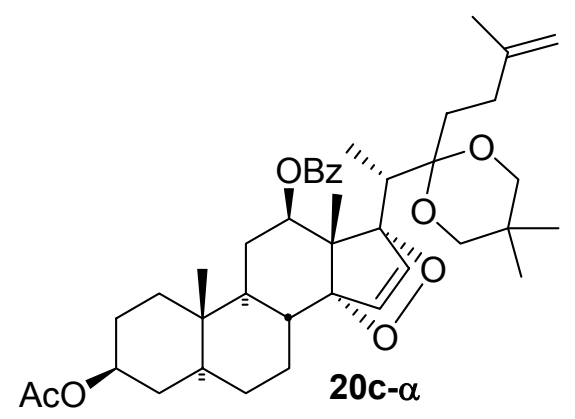

Endoperoxide 20c- $\alpha$. Prepared in 91\% yield following the above general procedure; white solid; mp 110 111 $\left(\mathrm{CH}_{2} \mathrm{Cl}_{2} / \mathrm{n}\right.$-Hexane); ${ }^{1} \mathrm{H} \mathrm{NMR}\left(300 \mathrm{MHz}, \mathrm{CDCl}_{3}\right) \delta 7.98(\mathrm{~d}, J=$ $7.3 \mathrm{~Hz}, 2 \mathrm{H}), 7.52-7.57(\mathrm{~m}, 1 \mathrm{H}), 7.38-7.43(\mathrm{~m}, 2 \mathrm{H}), 6.91(\mathrm{~d}, J=6.2 \mathrm{~Hz}, 1 \mathrm{H}), 6.32(\mathrm{~d}, J$ $=6.0 \mathrm{~Hz}, 1 \mathrm{H}), 5.47(\mathrm{dd}, J=11.1,4.5 \mathrm{~Hz}, 1 \mathrm{H}), 4.63-4.71(\mathrm{~m}, 1 \mathrm{H}), 4.59(\mathrm{~s}, 2 \mathrm{H}), 3.65(\mathrm{~d}$, $J=11.4 \mathrm{~Hz}, 1 \mathrm{H}), 3.40(\mathrm{~d}, J=11.1 \mathrm{~Hz}, 1 \mathrm{H}), 3.27-3.31(\mathrm{~m}, 2 \mathrm{H}), 3.19$ (d, $J=11.3 \mathrm{~Hz}$, 1H), $2.10-2.25(\mathrm{~m}, 3 \mathrm{H}), 1.98(\mathrm{~s}, 3 \mathrm{H}), 1.63(\mathrm{~s}, 3 \mathrm{H}), 1.19(\mathrm{~s}, 3 \mathrm{H}), 1.11(\mathrm{~s}, 3 \mathrm{H}), 0.81(\mathrm{~d}, J$ $=5.0 \mathrm{~Hz}, 3 \mathrm{H}), 0.80(\mathrm{~s}, 3 \mathrm{H}), 0.78(\mathrm{~s}, 3 \mathrm{H}) ;{ }^{13} \mathrm{C} \mathrm{NMR}\left(75 \mathrm{MHz}, \mathrm{CDCl}_{3}\right) \delta 170.4,165.2$, $145.9,138.6,133.0,132.1,130.6,129.2$, 128.4, 109.5, 101.0, 96.5, 96.3, 73.2, 72.8, 69.5, 
69.4, 65.2, 45.0, 43.7, 36.5, 35.5, 33.8, 33.6, 29.8, 29.0, 28.3, 27.2, 27.1, 26.3, 23.2, 22.7, 21.3, 14.2, 11.5, 9.2; HRMS (ESI) $\mathrm{C}_{41} \mathrm{H}_{56} \mathrm{O}_{8} \mathrm{Na}[\mathrm{M}+\mathrm{Na}]^{+}$calcd. 699.3873, found 699.3875 .

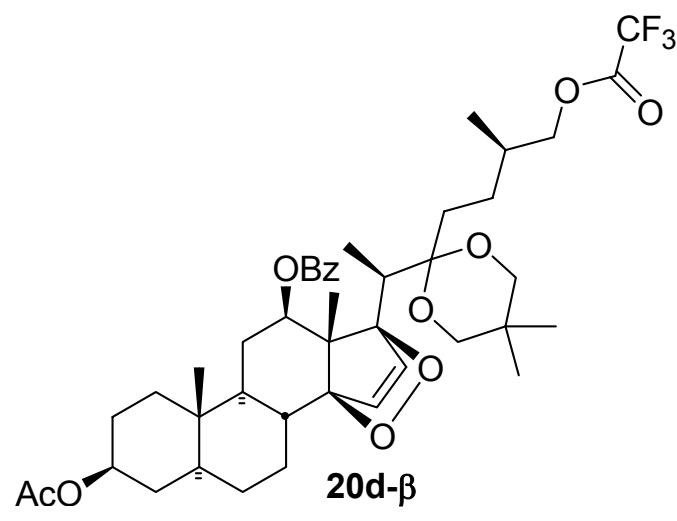

Endoperoxide 20d- $\beta$. Prepared in $81 \%$ yield following the above general procedure; oil; ${ }^{1} \mathrm{H}$ NMR (300 MHz, $\left.\mathrm{CDCl}_{3}\right) \delta 7.99$ (d, J=7.2 Hz, 2H), $7.53-7.58$ (m, 1H), $7.39-7.44$ (m, 2H), 6.9 (d, $J=6.0 \mathrm{~Hz}, 1 \mathrm{H}), 6.34(\mathrm{~d}, J=6.0 \mathrm{~Hz}, 1 \mathrm{H}), 5.47$ (dd, $J=11.1,4.5 \mathrm{~Hz}, 1 \mathrm{H})$, $4.65-4.72(\mathrm{~m}, 1 \mathrm{H}), 4.12(\mathrm{ddd}, J=19.2,10.7,5.9 \mathrm{~Hz}, 2 \mathrm{H}), 3.65(\mathrm{~d}, J=11.4 \mathrm{~Hz}, 1 \mathrm{H})$, $3.38(\mathrm{~d}, J=11.4 \mathrm{~Hz}, 1 \mathrm{H}), 3.27-3.30(\mathrm{~m}, 2 \mathrm{H}), 3.17(\mathrm{~d}, J=11.1 \mathrm{~Hz}, 1 \mathrm{H}), 2.22-2.27(\mathrm{~m}$, 1H), 1.99 (s, 3H), 1.19 (s, 3H), 1.10 (s, 3H), 0.90 (d, $J=6.6 \mathrm{~Hz}, 3 \mathrm{H}), 0.81$ (s, 3H), 0.79 (d, $J=7.0 \mathrm{~Hz}, 3 \mathrm{H}), 0.77(\mathrm{~s}, 3 \mathrm{H}) ;{ }^{13} \mathrm{C}$ NMR $\left(75 \mathrm{MHz}, \mathrm{CDCl}_{3}\right) \delta 170.5,165.2,138.5$, 133.1, 132.4, 130.7, 129.2, 128.4, 101.1, 96.6, 96.3, 73.2, 72.8, 69.6, 69.4, 65.4, 45.0, 43.7, 36.5, 35.5, 33.9, 33.7, 32.4, 29.0, 28.4, 27.2, 27.1, 26.4, 24.8, 23.2, 22.6, 21.3, 16.4, $14.2,11.5,10.4,9.2 ;{ }^{19} \mathrm{~F}$ NMR (282 MHz, $\left.\mathrm{CDCl}_{3}\right) \delta-75.03$; HRMS (ESI) $\mathrm{C}_{43} \mathrm{H}_{57} \mathrm{~F}_{3} \mathrm{O}_{10} \mathrm{Na}$ $[\mathrm{M}+\mathrm{Na}]^{+}$calcd. 813.3802, found 813.3802.

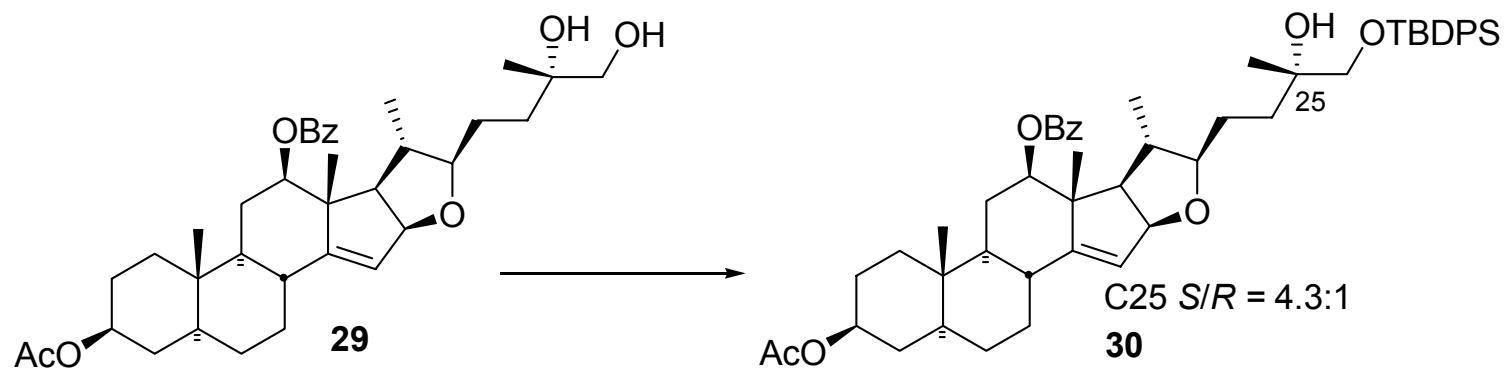


Silyl ether 30. To a solution of C25, 26-diol $\mathbf{2 9}^{4}(1.19 \mathrm{~g}, 0.0020 \mathrm{~mol})$, triethylamine (1.4 $\mathrm{mL}, 0.0100 \mathrm{~mol}, 5$ equiv), and $10 \mathrm{~mol} \%$ DMAP $(24.5 \mathrm{mg})$ in $\mathrm{CH}_{2} \mathrm{Cl}_{2}(13 \mathrm{~mL})$ was added TBDPSCl ( $1.3 \mathrm{~mL}, 0.0050 \mathrm{~mol}, 2.5$ equiv) at room temperature. The reaction was stirred for $36 \mathrm{~h}$. The reaction was diluted with EtOAc, worked up with water. The organic layer was washed with brine, dried over $\mathrm{Na}_{2} \mathrm{SO}_{4}$, filtered, and concentrated in vacuo. The crude residue was purified by flash chromatography $(\mathrm{EtOAc} / \mathrm{n}-\mathrm{Hexane}=1: 4)$ to give silyl ether 30 (1.65 g, $0.0020 \mathrm{~mol}, 99 \%)$ as colorless oil; ${ }^{1} \mathrm{H}$ NMR $\left(300 \mathrm{MHz}, \mathrm{CDCl}_{3}\right) \delta 8.04(\mathrm{~d}, J=$ $7.3 \mathrm{~Hz}, 2 \mathrm{H}), 7.63$ (d, $J=6.6 \mathrm{~Hz}, 4 \mathrm{H}), 7.53-7.58(\mathrm{~m}, 1 \mathrm{H}), 7.33-7.46(\mathrm{~m}, 8 \mathrm{H}), 5.44$ (s, $1 \mathrm{H}), 4.62-4.76(\mathrm{~m}, 2 \mathrm{H}), 3.43$ (s, 2H), 3.22 (bt, $J=7.6 \mathrm{~Hz}, 1 \mathrm{H}), 2.66$ (bs, $1 \mathrm{H}), 2.22(\mathrm{t}, J$ $=7.8 \mathrm{~Hz}, 1 \mathrm{H}), 2.02-2.13(\mathrm{~m}, 1 \mathrm{H}), 1.99(\mathrm{~s}, 3 \mathrm{H}), 1.22(\mathrm{~s}, 3 \mathrm{H}), 1.13(\mathrm{~s}, 3 \mathrm{H}), 1.05(\mathrm{~s}, 9 \mathrm{H})$, $0.88(\mathrm{~s}, 3 \mathrm{H}), 0.83(\mathrm{~d}, J=6.6 \mathrm{~Hz}, 3 \mathrm{H}) ;{ }^{13} \mathrm{C} \mathrm{NMR}\left(75 \mathrm{MHz}, \mathrm{CDCl}_{3}\right) \delta 170.5,165.9,157.0$, 135.6, 135.0, 133.2, 133.1, 133.0, 130.5, 129.7, 129.4, 128.4, 127.7, 120.2, 87.5, 86.0, 81.7, 73.2, 72.3, 59.6, 51.9, 51.8, 44.2, 41.1, 36.5, 35.9, 35.4, 34.1, 33.7, 29.5, 28.0, 27.2, 27.0, 26.9, 26.7, 23.5, 21.4, 19.3, 16.8, 15.9, 12.0; HRMS (ESI) $\mathrm{C}_{52} \mathrm{H}_{68} \mathrm{O}_{7} \mathrm{Si}[\mathrm{M}]^{+}$calcd. 833.4813 , found 833.4829 .

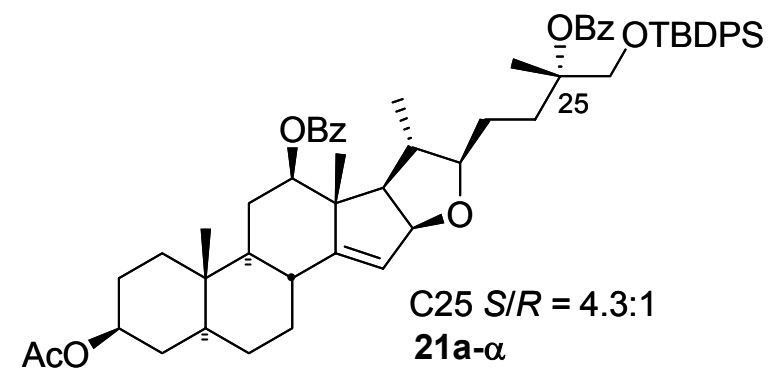

Tertiary benzoate 21a- $\alpha$. To a solution of silyl ether 30 (2.27 g, $0.0027 \mathrm{~mol}), \mathrm{Bz}_{2} \mathrm{O}$ (1.41 g, 0.0054 mol, 2 equiv), and $\mathrm{MgBr}_{2} . \mathrm{OEt}_{2}\left(1.41 \mathrm{~g}, 0.0054 \mathrm{~mol}\right.$, 2 equiv) in $\mathrm{CH}_{2} \mathrm{Cl}_{2}$ $(27 \mathrm{~mL})$ was added triethylamine $\left(1.14 \mathrm{~mL}, 0.0082 \mathrm{~mol}, 3.0\right.$ equiv) at $0{ }^{\circ} \mathrm{C}$. The reaction was stirred at room temperature for $2.5 \mathrm{~h}$. The reaction was diluted with EtOAc, worked up with water. The organic layer was washed with brine, dried over $\mathrm{Na}_{2} \mathrm{SO}_{4}$, filtered, and concentrated in vacuo. The crude residue was purified by flash column chromatography

\footnotetext{
${ }^{4}$ For preparation of diol see (a) Lee, S.; LaCour, T. G.; Lantrip, D.; Fuchs, P. L. Org. Lett. 2002, 4, 313. (b) Lee, S.; Fuchs, P. L. Org. Lett. 2002, 4, 317.
} 
$($ EtOAc/n-Hexane $=1: 4)$ to give tertiary benzoate $21 \mathrm{a}-\alpha(2.53 \mathrm{~g}, 0.0026 \mathrm{~mol}, 99 \%)$ as white solid; mp $86 \sim 87{ }^{\circ} \mathrm{C}\left(\mathrm{CH}_{2} \mathrm{Cl}_{2} / \mathrm{n}\right.$-Hexane); ${ }^{1} \mathrm{H}$ NMR (300 MHz, $\left.\mathrm{CDCl}_{3}\right) \delta 8.03$ (d, $J$ $=7.3 \mathrm{~Hz}, 2 \mathrm{H}), 7.94(\mathrm{~d}, J=7.5 \mathrm{~Hz}, 2 \mathrm{H}), 7.27-7.62(\mathrm{~m}, 16 \mathrm{H}), 5.46(\mathrm{~s}, 1 \mathrm{H}), 4.64-4.76$ (m, 3H), $3.89-3.97(\mathrm{~m}, 2 \mathrm{H}), 3.22-3.29(\mathrm{~m}, 1 \mathrm{H}), 2.19-2.24(\mathrm{~m}, 2 \mathrm{H}), 2.07-2.14(\mathrm{~m}$, 2H), 2.00 (s, 3H), 1.56 (s, 3H), 1.21 (s, 3H), 0.99 (s, 9H), 0.88 (s, 3H), 0.81 (d, J=6.4 Hz, $3 \mathrm{H}) ;{ }^{13} \mathrm{C}$ NMR $\left(75 \mathrm{MHz}, \mathrm{CDCl}_{3}\right) \delta 170.3,165.8,165.3,156.8,135.5,133.2,132.8,132.3$, 131.6, 130.4, 129.5, 129.4, 128.3, 128.0, 127.5, 120.2, 87.2, 85.9, 84.7, 81.7, 73.1, 66.9, $59.9,51.9,51.7,44.1,40.9,36.5,35.8,34.5,34.1,33.7,32.8,29.4,28.0,27.2,26.7,26.6$, 25.2, 21.4, 21.2, 20.6, 19.2, 17.0, 16.0, 11.9; HRMS (ESI) $\mathrm{C}_{59} \mathrm{H}_{72} \mathrm{O}_{8} \mathrm{SiNa}[\mathrm{M}+\mathrm{Na}]^{+}$calcd. 959.4894, found 959.4899 .

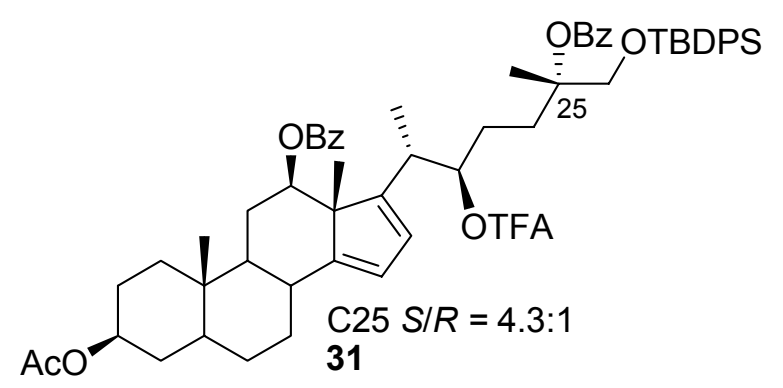

Dienyl trifluoroacetate 31. To a solution of tertiary benzoate $21 \mathrm{a}-\alpha(1.63 \mathrm{~g}, 1.74 \mathrm{mmol})$ and 2,6-di-tert-butyl-4-methyl pyridine (714.6 $\mathrm{mg}, 3.48 \mathrm{mmol}, 2$ equiv) in $\mathrm{CH}_{2} \mathrm{Cl}_{2}$ (12 $\mathrm{mL})$ was added freshly prepared TFAT $(0.8 \mathrm{~mL}, 5.22 \mathrm{mmol}, 3.0$ equiv $)$ at $-30{ }^{\circ} \mathrm{C}$. The reaction was stirred for $40 \mathrm{~min}$. at $-30 \sim-40{ }^{\circ} \mathrm{C}$. The reaction was quenched by adding sat. $\mathrm{NaHCO}_{3}$ and diluted with EtOAc. The organic layer was washed with brine, dried over $\mathrm{Na}_{2} \mathrm{SO}_{4}$, filtered, and concentrated in vacuo. The crude residue was purified by flash column chromatography (EtOAc/n-Hexane $=1: 4)$ to give dienyl trifluoroacetate 31 (1.75 g, 1.69 mmol, 97\%) as white solid; mp $92 \sim 93{ }^{\circ} \mathrm{C}\left(\mathrm{CH}_{2} \mathrm{Cl}_{2} / \mathrm{n}\right.$-Hexane); ${ }^{1} \mathrm{H}$ NMR (300 $\left.\mathrm{MHz}, \mathrm{CDCl}_{3}\right) \delta 8.06(\mathrm{~d}, J=7.5 \mathrm{~Hz}, 2 \mathrm{H}), 7.92(\mathrm{~d}, J=7.3 \mathrm{~Hz}, 2 \mathrm{H}), 7.61-7.65(\mathrm{~m}, 4 \mathrm{H})$, $7.30-7.55$ (m, 12H), 6.16 (s, C25-S, 1H), 6.12 (s, C25-R, 1H), 5.88 (s, C25-S, 1H), 5.84 (s, C25-R, 1H), $5.15-5.17(\mathrm{~m}, 1 \mathrm{H}), 4.68-4.76(\mathrm{~m}, 1 \mathrm{H}), 4.44(\mathrm{dd}, J=10.8,3.5 \mathrm{~Hz}, 1 \mathrm{H})$, $3.97(\mathrm{~d}, J=10.4 \mathrm{~Hz}, 1 \mathrm{H}), 3.88(\mathrm{~d}, J=10.5 \mathrm{~Hz}, 1 \mathrm{H}), 2.95(\mathrm{t}, J=6.7 \mathrm{~Hz}, 1 \mathrm{H}), 2.15-2.24$ (m, 2H), 2.02 (s, 3H), 1.51 (s, 3H), $1.02(\mathrm{~s}, 9 \mathrm{H}), 0.95$ (s, 3H), 0.88 (d, J=6.9 Hz, 3H); 
${ }^{13} \mathrm{C}$ NMR $\left(75 \mathrm{MHz}, \mathrm{CDCl}_{3}\right) \delta 170.4,165.4,165.2,156.5,155.8,135.5,133.1,133.0$, $132.9,132.5,131.3,130.2,129.7,129.4,129.3,128.4,128.1,127.6,126.3,120.8,83.9$, $82.5,79.3,73.2,66.9,56.9,53.1,44.2,36.9,35.8,35.5,34.6,33.7,31.4,29.1,28.1,27.3$, $27.2,26.6,25.2,24.2,21.3,21.1,19.1,18.0,13.5,12.1 ;{ }^{19} \mathrm{~F} \mathrm{NMR}\left(282 \mathrm{MHz}, \mathrm{CDCl}_{3}\right) \delta$ 74.85; HRMS (ESI) $\mathrm{C}_{61} \mathrm{H}_{71} \mathrm{~F}_{3} \mathrm{O}_{9} \mathrm{SiNa}[\mathrm{M}+\mathrm{Na}]^{+}$calcd. 1055.4717, found 1055.4706.

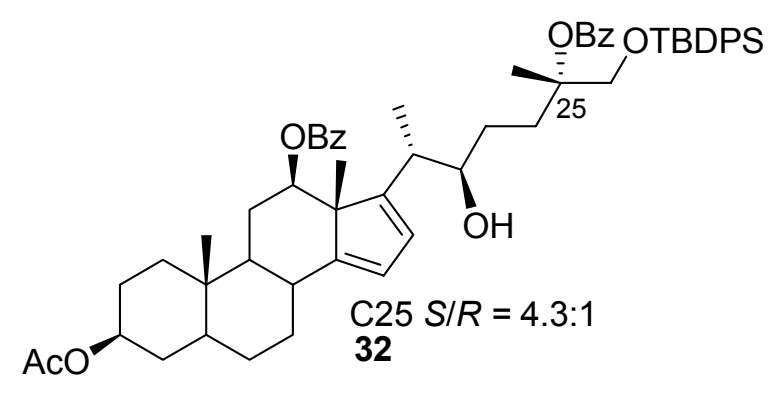

Secondary alcohol 32. Dienyl trifluoroacetate $31(1.67 \mathrm{~g}, 1.62 \mathrm{mmol})$ was stirred with $\mathrm{Na}_{2} \mathrm{CO}_{3}$ (687.4 mg, $6.49 \mathrm{mmol}$, 4 equiv) in 8:1:1 mixture of THF, water, and $\mathrm{MeOH}$ at room temperature for $7 \mathrm{~h}$. The reaction was extracted with EtOAc three times. The extract was washed with brine, dried over $\mathrm{Na}_{2} \mathrm{SO}_{4}$, filtered, and concentrated in vacuo. The crude residue was purified by flash column chromatography (EtOAc/n-Hexane $=$ 1:4) to give secondary alcohol $32(1.41 \mathrm{~g}, 1.51 \mathrm{mmol}, 93 \%)$ as white solid; $\mathrm{mp} 85 \sim 87$ ${ }^{\circ} \mathrm{C}\left(\mathrm{CH}_{2} \mathrm{Cl}_{2} / \mathrm{n}\right.$-Hexane); ${ }^{1} \mathrm{H}$ NMR (300 MHz, $\left.\mathrm{CDCl}_{3}\right) \delta 8.04(\mathrm{~d}, J=7.2 \mathrm{~Hz}, 2 \mathrm{H}), 7.93(\mathrm{~d}, J$ $=7.2 \mathrm{~Hz}, 2 \mathrm{H}), 7.61-7.64(\mathrm{~m}, 4 \mathrm{H}), 7.27-7.53(\mathrm{~m}, 12 \mathrm{H}), 6.17(\mathrm{~d}, J=1.8 \mathrm{~Hz}, 1 \mathrm{H}), 6.15$ (d, $J=1.9 \mathrm{~Hz}, \mathrm{C} 25-R, 1 \mathrm{H}), 5.94(\mathrm{~s}, 1 \mathrm{H}), 4.64-4.73(\mathrm{~m}, 1 \mathrm{H}), 4.44$ (dd, $J=11.1,4.1 \mathrm{~Hz}$, $1 \mathrm{H}), 3.96(\mathrm{~d}, J=10.4 \mathrm{~Hz}, 1 \mathrm{H}), 3.90(\mathrm{~d}, J=8.2 \mathrm{~Hz}, 1 \mathrm{H}), 3.56(\mathrm{bt}, J=8.2 \mathrm{~Hz}, 1 \mathrm{H}), 2.54$ (quintet, $J=7.8 \mathrm{~Hz}, 1 \mathrm{H}), 2.13-2.25(\mathrm{~m}, 2 \mathrm{H}), 2.01$ (s, 3H), $1.53(\mathrm{~s}, 3 \mathrm{H}), 1.25(\mathrm{~s}, 3 \mathrm{H})$, 1.00 (s, 9H), 0.92 (s, 3H), 0.77 (d, $J=6.7 \mathrm{~Hz}, 3 \mathrm{H}) ;{ }^{13} \mathrm{C} \mathrm{NMR}\left(75 \mathrm{MHz}, \mathrm{CDCl}_{3}\right) \delta 170.5$, 165.5, 165.4, 160.0, 156.0, 135.6, 133.3, 133.0, 132.4, 131.7, 130.4, 129.6, 129.4, 129.3, $128.4,128.1,127.6,124.9,120.7,84.8,79.1,75.9,73.3,67.2,57.0,53.1,44.2,39.3,36.9$, 35.8, 34.7, 33.8, 31.9, 29.1, 28.1, 27.6, 27.3, 27.2, 26.7, 21.4, 21.3, 19.6, 19.2, 14.3, 12.1; HRMS (ESI) $\mathrm{C}_{59} \mathrm{H}_{72} \mathrm{O}_{8} \mathrm{SiNa}[\mathrm{M}+\mathrm{Na}]^{+}$calcd. 959.4894, found 959.4902 . 


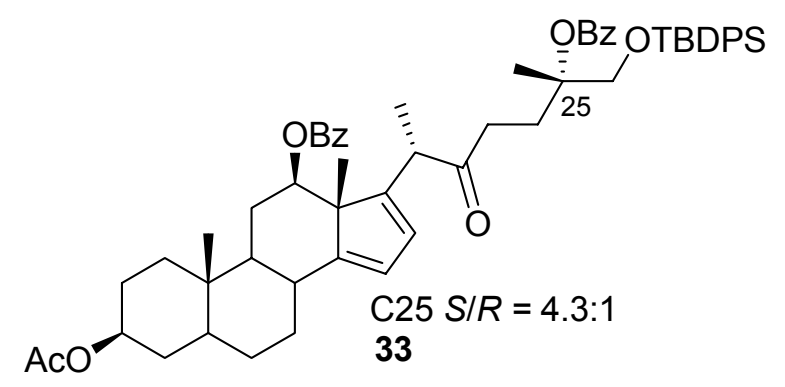

Ketone 33. To a solution of freshly distilled DMSO (1.5 mL, $20.84 \mathrm{mmol}, 3$ equiv) in $\mathrm{CH}_{2} \mathrm{Cl}_{2}(5 \mathrm{~mL})$ was added TFAA $\left(2.5 \mathrm{~mL}, 17.36 \mathrm{mmol}, 2.5\right.$ equiv) at $-78{ }^{\circ} \mathrm{C}$. The solution was stirred for $20 \mathrm{~min}$. at $-78{ }^{\circ} \mathrm{C}$. To the solution, secondary alcohol $32(6.51 \mathrm{~g}$, $6.95 \mathrm{mmol})$ in $\mathrm{CH}_{2} \mathrm{Cl}_{2}(64 \mathrm{~mL})$ was added dropwise using a dropping funnel for $20 \mathrm{~min}$. at $-78{ }^{\circ} \mathrm{C}$. The reaction was stirred for $40 \mathrm{~min}$. at $-78{ }^{\circ} \mathrm{C}$, and allowed to warm to room temperature. The reaction was stirred for additional $6 \mathrm{~h}$ at room temperature. After $6 \mathrm{~h}$, the reaction was diluted with EtOAc, worked up with water, washed with brine, and dried over $\mathrm{Na}_{2} \mathrm{SO}_{4}$. After removal of solvent, the crude residue was purified by flash column chromatography (EtOAc/n-Hexane = 1:8) to provide ketone 33 (6.50 g, $6.94 \mathrm{mmol}, 99 \%)$ as white solid; mp $77 \sim 79{ }^{\circ} \mathrm{C}\left(\mathrm{CH}_{2} \mathrm{Cl}_{2} / \mathrm{n}\right.$-Hexane); ${ }^{1} \mathrm{H} \mathrm{NMR}\left(300 \mathrm{MHz}, \mathrm{CDCl}_{3}\right) \delta 8.05$ (d, $J=7.3 \mathrm{~Hz}, 2 \mathrm{H}), 7.90$ (d, $J=7.5 \mathrm{~Hz}, 2 \mathrm{H}), 7.50-7.61$ (m, 6H), $7.25-7.43$ (m, 10H), 6.04 (d, $J=1.6 \mathrm{~Hz}, 1 \mathrm{H}), 5.87(\mathrm{~s}, 1 \mathrm{H}), 4.64-4.71(\mathrm{~m}, 1 \mathrm{H}), 4.39$ (dd, $J=11.1,4.2 \mathrm{~Hz}, 1 \mathrm{H})$, $3.89(\mathrm{~s}, 2 \mathrm{H}), 3.43(\mathrm{q}, J=6.6 \mathrm{~Hz}, 1 \mathrm{H}), 2.43-2.58(\mathrm{~m}, 1 \mathrm{H}), 2.22-2.41(\mathrm{~m}, 2 \mathrm{H}), 2.02-$ 2.18 (m, 2H), 2.00 (s, 3H), 1.52 (s, 3H), 1.14 (s, 3H), 0.99 (s, 12H), $0.89(\mathrm{~s}, 3 \mathrm{H}) ;{ }^{13} \mathrm{C}$ NMR (75 MHz, $\left.\mathrm{CDCl}_{3}\right) \delta 209.3,170.5,165.5,165.2,156.4,154.4,135.5,133.1,132.5$, $131.4,130.3,129.6,129.43,129.38,128.5,128.1,127.6,127.5,121.0,84.1,78.9,73.2$, 67.1, 56.8, 52.9, 46.0, 44.2, 36.9, 35.7, 34.7, 34.2, 33.7, 30.5, 29.1, 28.0, 27.2, 27.1, 26.7, 21.5, 21.3, 19.2, 17.8, 14.2, 12.1; HRMS (ESI) $\mathrm{C}_{59} \mathrm{H}_{70} \mathrm{O}_{8} \mathrm{SiNa}[\mathrm{M}+\mathrm{Na}]^{+}$calcd. 957.4738, found 957.4757 . 


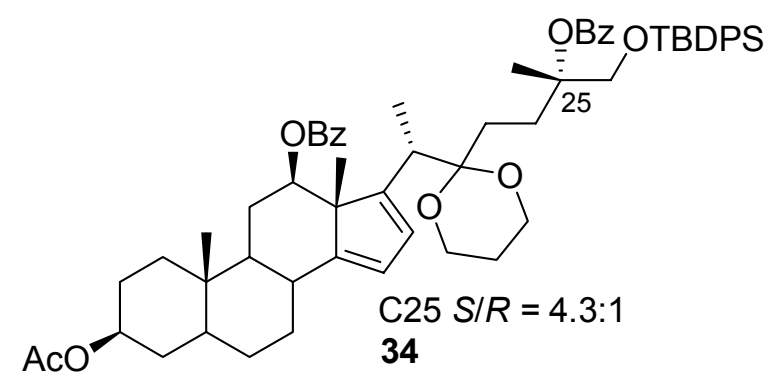

Ketal 34. To a solution of ketone $33(6.50 \mathrm{~g}, 6.95 \mathrm{mmol})$ and $\mathrm{Sc}(\mathrm{OTf})_{3}(342.0 \mathrm{mg}, 0.695$ mmol, 0.1 equiv) in $\mathrm{CH}_{3} \mathrm{CN}(60 \mathrm{~mL})$ was added 1,3-propanediol $(5.0 \mathrm{~mL}, 69.5 \mathrm{mmol}, 10$ equiv), followed by $\mathrm{HC}(\mathrm{OMe})_{3}(7.6 \mathrm{~mL}, 69.5 \mathrm{mmol}, 10$ equiv). The reaction was stirred at room temperature for $17 \mathrm{~h}$. The reaction was diluted with EtOAc, worked up with water, washed with brine, and dried over $\mathrm{Na}_{2} \mathrm{SO}_{4}$. After removal of solvent, the crude residue was treated with $\mathrm{Ac}_{2} \mathrm{O}(6.6 \mathrm{~mL}, 69.5 \mathrm{mmol}, 10$ equiv) and DMAP $(84.9 \mathrm{mg}$, $0.695 \mathrm{mmol}, 0.1$ equiv) in pyridine $(69 \mathrm{~mL})$ at $\mathrm{r} . \mathrm{f}$ for $4 \mathrm{~h}$. As the reaction was completed, pyridine was removed by $10 \% \mathrm{HCl}$ and EtOAc. The organic layer was neutralized by sat. $\mathrm{NaHCO}_{3}$, washed with brine, dried over $\mathrm{Na}_{2} \mathrm{SO}_{4}$ and evaporated in vacuo. The residue was purified by flash column chromatography $(\mathrm{EtOAc} / \mathrm{n}-\mathrm{Hexane}=1: 8)$ to provide ketal 34 (6.26 g, 6.30 mmol, 91\%) as white solid; mp $102 \sim 103{ }^{\circ} \mathrm{C}\left(\mathrm{CH}_{2} \mathrm{Cl}_{2} / \mathrm{n}-\mathrm{Hexane}\right) ;{ }^{1} \mathrm{H}$ NMR (300 MHz, $\left.\mathrm{CDCl}_{3}\right) \delta 8.07-8.13(\mathrm{~m}, 2 \mathrm{H}), 8.00(\mathrm{~d}, J=7.2 \mathrm{~Hz}, 2 \mathrm{H}), 7.67-7.72(\mathrm{~m}$, 4H), $7.54-7.61(\mathrm{~m}, 1 \mathrm{H}), 7.26-7.48(\mathrm{~m}, 11 \mathrm{H}), 6.42(\mathrm{~s}, 1 \mathrm{H}), 6.33(\mathrm{~s}, \mathrm{C} 25-R, 1 \mathrm{H}), 6.01(\mathrm{~s}$, 1H), $4.73-4.80$ (m, 1H), 4.51 (dd, $J=11.1,4.2 \mathrm{~Hz}, 1 \mathrm{H}), 4.11$ (d, $J=10.4 \mathrm{~Hz}, 1 \mathrm{H}), 4.03$ (d, $J=10.1 \mathrm{~Hz}, \mathrm{C} 25-R, 1 \mathrm{H}), 3.74-3.92(\mathrm{~m}, 4 \mathrm{H}), 3.61-3.64(\mathrm{~m}, 1 \mathrm{H}), 2.85-2.92(\mathrm{~m}$, $1 \mathrm{H}), 2.23-2.36(\mathrm{~m}, 3 \mathrm{H}), 2.08(\mathrm{~s}, 3 \mathrm{H}), 1.44(\mathrm{~s}, 3 \mathrm{H}), 1.08(\mathrm{~s}, 9 \mathrm{H}), 1.06(\mathrm{~s}, 3 \mathrm{H}), 0.92(\mathrm{~s}$, $3 \mathrm{H}), 0.91(\mathrm{~d}, J=7.3 \mathrm{~Hz}, 3 \mathrm{H}) ;{ }^{13} \mathrm{C}$ NMR $\left(75 \mathrm{MHz}, \mathrm{CDCl}_{3}\right) \delta 170.5,165.45,165.42,158.2$, 154.1, 135.59, 135.57, 133.4, 133.3, 132.8, 131.6, 130.6, 129.7, 129.4, 129.3, 128.3, $128.1,127.6,126.6,121.4,101.4,84.4,79.7,73.3,67.1,59.3,59.0,57.1,53.3,44.3,37.6$, $36.9,35.7,34.6,33.8,29.8,29.2,28.2,27.5,27.2,26.7,25.1,21.5,21.4,21.3,19.2,17.6$, 13.9, 12.2; HRMS (ESI) $\mathrm{C}_{62} \mathrm{H}_{76} \mathrm{O} 99 \mathrm{SiNa}[\mathrm{M}+\mathrm{Na}]^{+}$calcd. 1015.5156, found 1015.5145. 


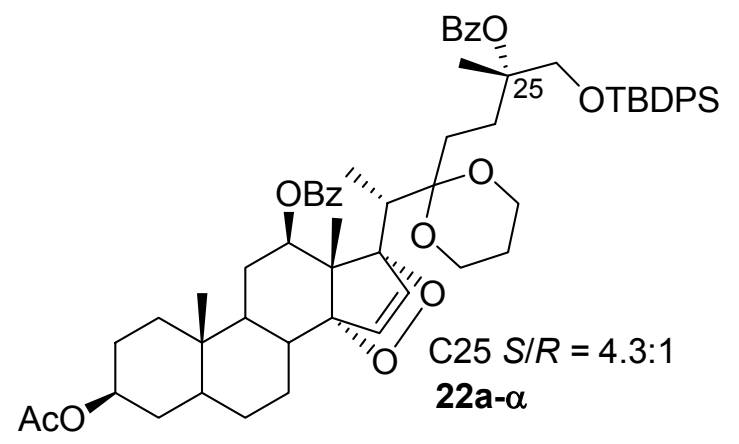

Endoperoxide 22a- $\alpha$. A solution of ketal 34 (2.04 g, $2.05 \mathrm{mmol})$ and 5,10,15,20tetraphenyl-21 $\mathrm{H}, 23 \mathrm{H}$-porphine, TPP (2.1 mg, $0.0034 \mathrm{mmol}, 0.0017$ equiv) in $\mathrm{CH}_{2} \mathrm{Cl}_{2}$ (20 $\mathrm{mL}$ ) was purged with oxygen at $-78{ }^{\circ} \mathrm{C}$ for $5 \mathrm{~min}$. The reaction was stirred with irradiation by a sun lamp (275W RS-M) at $-78^{\circ} \mathrm{C}$ for $1.5 \mathrm{~h}$. The reaction mixture was evaporated under reduced pressure at room temperature. TPP was removed by a short $\mathrm{Al}_{2} \mathrm{O}_{3}(\sim 150$ mesh, activated, neutral, Brockmann I) pad $(5$ x $5 \mathrm{~cm}$, EtOAc/n-Hexane $=$ 5:95) to give endoperoxide $22 \mathrm{a}-\alpha(2.02 \mathrm{~g}, 1.97 \mathrm{mmol}, 96 \%)$ as white solid; $\mathrm{mp} 89 \sim 91$ ${ }^{\circ} \mathrm{C} ;{ }^{1} \mathrm{H}$ NMR $\left(300 \mathrm{MHz}, \mathrm{CDCl}_{3}\right) \delta 7.92-8.01$ (m, 4H), 7.62 - 7.64 (m, 4H), 7.49 - 7.60 $(\mathrm{m}, 1 \mathrm{H}), 7.26-7.44(\mathrm{~m}, 11 \mathrm{H}), 7.02(\mathrm{~d}, J=6.0 \mathrm{~Hz}, 1 \mathrm{H}), 6.98(\mathrm{~d}, J=5.9 \mathrm{~Hz}, \mathrm{C} 25-R, 1 \mathrm{H})$, $6.22(\mathrm{~d}, J=5.9 \mathrm{~Hz}, 1 \mathrm{H}), 5.59$ (dd, $J=11.1,4.5 \mathrm{~Hz}, 1 \mathrm{H}), 4.67-4.74$ (m, 1H), $3.81-3.98$ $(\mathrm{m}, 3 \mathrm{H}), 3.63-3.79(\mathrm{~m}, 3 \mathrm{H}), 2.80(\mathrm{q}, J=4.7 \mathrm{~Hz}, 1 \mathrm{H}), 2.06-2.27(\mathrm{~m}, 3 \mathrm{H}), 2.01(\mathrm{~s}, 3 \mathrm{H})$, $1.39(\mathrm{~s}, 3 \mathrm{H}), 1.21(\mathrm{~s}, 3 \mathrm{H}), 1.02(\mathrm{~s}, 9 \mathrm{H}), 0.92(\mathrm{~d}, J=8.3 \mathrm{~Hz}, 3 \mathrm{H}), 0.86(\mathrm{~s}, 3 \mathrm{H}) ;{ }^{13} \mathrm{C} \mathrm{NMR}$ $\left(75 \mathrm{MHz}, \mathrm{CDCl}_{3}\right) \delta 170.4,165.5,165.3,135.60,135.57,133.4,133.3,132.8,132.4$, $131.6,130.7,130.6,129.6,129.5,129.3,128.3,128.1,127.6,101.5,97.0,96.9,84.4,73.2$, $72.3,66.8,64.5,58.7,45.2,43.8,36.5,35.5,34.6,33.8,28.8,28.4,27.2,26.7,26.5,25.2$, 24.3, 21.5, 21.3, 19.2, 14.0, 11.5, 10.0; HRMS (ESI) $\mathrm{C}_{62} \mathrm{H}_{76} \mathrm{O}_{11} \mathrm{Si}[\mathrm{M}+\mathrm{Na}]^{+}$calcd. 1047.5055, found 1047.5056.

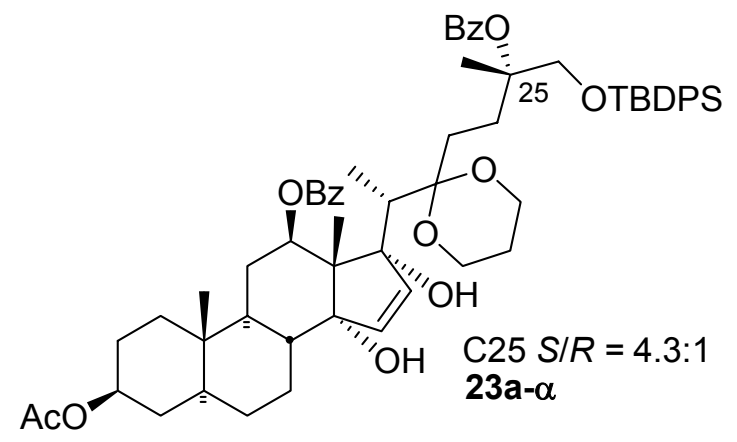


Diol 23a- $\alpha$. To a slurry of endoperoxide 22a- $\alpha(2.02 \mathrm{~g}, 1.97 \mathrm{mmol})$ and activated $\mathrm{Zn}(1.3$ g, $19.7 \mathrm{mmol}, 10$ equiv) in $\mathrm{CH}_{2} \mathrm{Cl}_{2}(20 \mathrm{~mL})$ was added $\mathrm{AcOH}(0.22 \mathrm{~mL}, 3.94 \mathrm{mmol}, 2$ equiv) dropwise at $0{ }^{\circ} \mathrm{C}$. The reaction was stirred at room temperature for $40 \mathrm{~min}$. After the reaction was completed, $\mathrm{Zn}$ was removed by Celite pad. The reaction mixture was quenched with sat. $\mathrm{NaHCO}_{3} / \mathrm{EtOAc}$, washed with brine, dried over $\mathrm{Na}_{2} \mathrm{SO}_{4}$ and the solvent was removed under reduced pressure. Flash column chromatography (EtOAc/nHexane $=1: 2)$ provided diol 23a- $\alpha(1.74 \mathrm{~g}, 1.69 \mathrm{mmol}, 86 \%)$ as white solid; $\mathrm{mp} 95 \sim 96$ ${ }^{\circ} \mathrm{C} ;{ }^{1} \mathrm{H}$ NMR $\left(300 \mathrm{MHz}, \mathrm{CDCl}_{3}\right) \delta 8.04(\mathrm{~d}, J=7.5 \mathrm{~Hz}, 2 \mathrm{H}), 7.9(\mathrm{~d}, J=7.6 \mathrm{~Hz}, 2 \mathrm{H}), 7.58$ $-7.66(\mathrm{~m}, 5 \mathrm{H}), 7.27-7.50(\mathrm{~m}, 11 \mathrm{H}), 7.14(\mathrm{~d}, J=6.0 \mathrm{~Hz}, 1 \mathrm{H}), 6.18(\mathrm{~d}, J=6.2 \mathrm{~Hz}, 1 \mathrm{H})$, $6.08(\mathrm{dd}, J=10.1,5.7 \mathrm{~Hz}, 1 \mathrm{H}), 4.67-4.74(\mathrm{~m}, 1 \mathrm{H}), 4.40(\mathrm{~s}, \mathrm{C} 25-R, 1 \mathrm{H}), 4.27(\mathrm{~s}, 1 \mathrm{H})$, $4.10(\mathrm{~s}, 1 \mathrm{H}), 4.08(\mathrm{~s}, \mathrm{C} 25-R, 1 \mathrm{H}), 3.76-4.05(\mathrm{~m}, 7 \mathrm{H}), 2.48-2.56(\mathrm{~m}, 1 \mathrm{H}), 2.08-2.17$ (m, 4H), 2.01 (s, 3H), 1.60 (s, 3H), 1.14 (s, 3H), 1.04 (s, 9H), 0.93 (d, J=7.3 Hz, 3H), $0.90(\mathrm{~s}, 3 \mathrm{H}) ;{ }^{13} \mathrm{C} \mathrm{NMR}\left(75 \mathrm{MHz}, \mathrm{CDCl}_{3}\right) \delta 170.5,165.5,165.3,140.8,135.6,135.5$, 134.6, 133.2, 133.1, 132.6, 132.5, 131.4, 131.1, 129.8, 129.7, 129.6, 129.3, 128.2, 128.1, $127.7,105.2,90.3,87.8,84.3,73.5,72.2,67.0,59.0,55.8,45.4,44.2,40.3,36.3,35.7$, 35.0, 34.6, 33.9, 29.6, 28.5, 27.3, 26.8, 26.3, 25.2, 24.4, 21.9, 21.6, 21.4, 19.3, 15.5, 11.6, 10.8; HRMS (ESI) $\mathrm{C}_{62} \mathrm{H}_{78} \mathrm{O}_{11} \mathrm{Si}[\mathrm{M}+\mathrm{Na}]^{+}$calcd. 1049.5211, found 1049.5229.

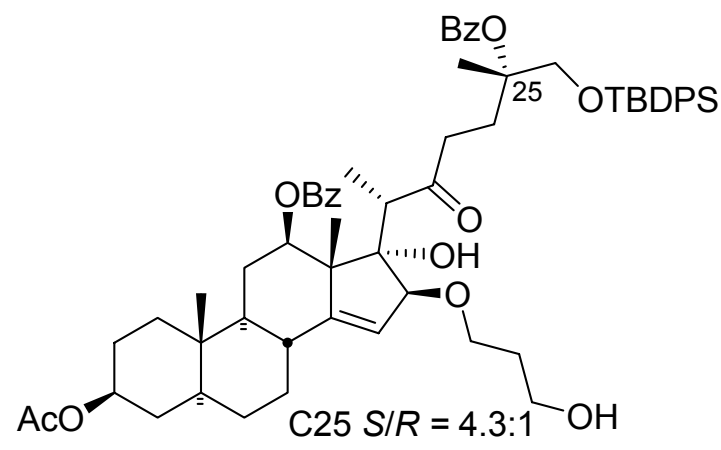

25

Hydroxypropyl ether 25. A solution of diol 23a- $\alpha$ (1.09 g, $1.06 \mathrm{mmol})$ and DDQ (2.4 mg, $0.01 \mathrm{mmol}, 0.01$ equiv) in aqueous $\mathrm{CH}_{3} \mathrm{CN}\left(55 \mathrm{~mL}, \mathrm{CH}_{3} \mathrm{CN} / \mathrm{H}_{2} \mathrm{O}=10: 1\right)$ was stirred at room temperature for $5 \mathrm{~h}$. The reaction mixture was worked up with sat. $\mathrm{NaHCO}_{3} / \mathrm{EtOAc}$, washed with brine, dried over $\mathrm{Na}_{2} \mathrm{SO}_{4}$ and the solvent was removed under reduced pressure. Flash column chromatography $($ EtOAc/n-Hexane $=1: 2)$ provided hydroxypropyl ether 25 (937.4 mg, $0.91 \mathrm{mmol}, 86 \%$ ) as white solid; mp $115 \sim 118{ }^{\circ} \mathrm{C} ;{ }^{1} \mathrm{H}$ 
NMR (300 MHz, $\left.\mathrm{CDCl}_{3}\right) \delta 8.00(\mathrm{~d}, J=7.3 \mathrm{~Hz}, 2 \mathrm{H}), 7.90(\mathrm{~d}, J=7.6 \mathrm{~Hz}, 2 \mathrm{H}), 7.41-7.59$ (m, 6H), $7.20-7.39$ (m, 10H), $5.62(\mathrm{dd}, J=11.6,4.5 \mathrm{~Hz}, 1 \mathrm{H}), 5.57$ (s, 1H), $4.63-4.66$ (m, 1H), $4.02(\mathrm{~s}, 1 \mathrm{H}), 4.01(\mathrm{~s}, \mathrm{C} 25-R, 1 \mathrm{H}), 3.75-3.88(\mathrm{~m}, 3 \mathrm{H}), 3.41-3.58(\mathrm{~m}, 3 \mathrm{H}), 3.26$ -3.33 (m, 1H), 2.92 (q, $J=7.0 \mathrm{~Hz}, 1 \mathrm{H}), 2.50-2.68(\mathrm{~m}, 2 \mathrm{H}), 2.26$ (t, $J=7.9 \mathrm{~Hz}, 1 \mathrm{H})$, 1.99 (s, 3H), 1.60 (s, 3H), 1.21 (s, 3H). 0.98 (s, 9H), 0.86 (d, $J=5.9 \mathrm{~Hz}, 3 \mathrm{H}), 0.82$ (s, $3 \mathrm{H}) ;{ }^{13} \mathrm{C}$ NMR $\left(75 \mathrm{MHz}, \mathrm{CDCl}_{3}\right) \delta 216.8,170.5,165.5,164.7,158.6,135.53,135.49$, 133.0, 132.9, 132.8, 132.6, 131.1, 131.0, 129.7, 129.6, 129.4, 128.2, 127.6, 117.7, 90.6, 84.7, 84.1, 73.3, 71.9, 67.1, 67.0, 61.1, 55.5, 49.2, 45.1, 43.6, 36.5, 35.7, 35.5, 34.7, 33.7, $32.6,29.5,28.8,27.9,27.3,27.1,26.7,22.1,19.2,17.1,12.1,11.7$; HRMS (ESI) $\mathrm{C}_{62} \mathrm{H}_{78} \mathrm{O}_{11} \mathrm{SiNa}[\mathrm{M}+\mathrm{Na}]^{+}$calcd. 1049.5211, found 1049.5199.

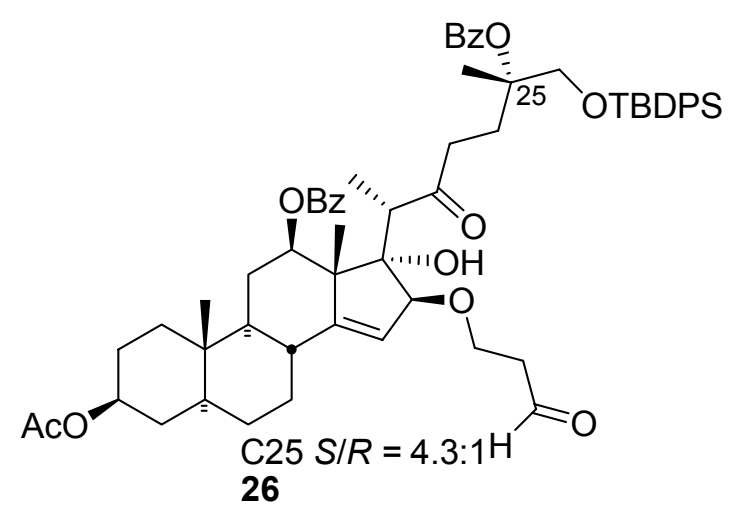

Propionaldehyde 26. Solid TPAP, ${ }^{5}$ tetrapropylammonium perruthenate $(9.5 \mathrm{mg}, 0.03$ mmol, 0.05 equiv) was added in one portion to a stirred solution of hydroxypropyl ether 25 (555.6 mg, $0.5408 \mathrm{mmol}$ ), NMO, 4-methylmorpholine $N$-oxide (95.0 mg, 0.8112 mmol, 1.5 equiv) and powdered molecular sieves (270.4 mg, $4 \AA$, activated) in $\mathrm{CH}_{2} \mathrm{Cl}_{2}$ (5 $\mathrm{mL}$ ) at room temperature. $0.5 \mathrm{~mL}$ of $\mathrm{CH}_{3} \mathrm{CN}$ was added to the solution. On completion the reaction mixture was filtered through a short silicagel pad to provide propionaldehyde 26 (549.0 mg, $0.5354 \mathrm{mmol}, 99 \%$ ) as white solid; mp $117 \sim 119{ }^{\circ} \mathrm{C}$; ${ }^{1} \mathrm{H}$ NMR (300 MHz, $\left.\mathrm{CDCl}_{3}\right) \delta 9.49$ (s, 1H), 9.47 (s, C25-R, 1H), 8.00 (d, $\left.J=7.2 \mathrm{~Hz}, 2 \mathrm{H}\right), 7.90$ (d, $J=7.5 \mathrm{~Hz}$, 2H), $7.41-7.59(\mathrm{~m}, 6 \mathrm{H}), 7.21-7.39(\mathrm{~m}, 10 \mathrm{H}), 5.62(\mathrm{dd}, J=11.6,4.5 \mathrm{~Hz}, 1 \mathrm{H}), 5.56(\mathrm{~s}$, 1H), $4.63-4.67(\mathrm{~m}, 1 \mathrm{H}), 4.03(\mathrm{~s}, 1 \mathrm{H}), 3.78-3.93(\mathrm{~m} \mathrm{3H}), 3.66-3.73(\mathrm{~m}, 1 \mathrm{H}), 3.37-$ $3.44(\mathrm{~m}, 1 \mathrm{H}), 2.89-2.95(\mathrm{~m}, 1 \mathrm{H}), 2.58-2.66(\mathrm{~m}, 1 \mathrm{H}), 2.31-2.57(\mathrm{~m}, 3 \mathrm{H}), 2.24-2.26$

\footnotetext{
${ }^{5}$ Ley, S. V.; Norman, J.; Griffith, W. P.; Marsden, S. P. Synthesis, 1994, 639.
} 
(m, 2H), 1.99 (s, 3H), 1.60 (s, 3H), 1.24 (s, 3H), 0.98 (s, 9H), 0.85 (d, J=7.5 Hz, 3H), $0.82(\mathrm{~s}, 3 \mathrm{H}) ;{ }^{13} \mathrm{C} \mathrm{NMR}\left(75 \mathrm{MHz}, \mathrm{CDCl}_{3}\right) \delta 217.0,200.4,170.5,165.3,164.8,159.1$, 135.6, 133.05, 133.0, 132.7, 132.6, 131.2, 131.0, 129.7, 129.6, 129.4, 128.2, 127.6, 127.1, $117.4,90.2,84.6,84.0,76.0,73.3,71.9,67.2,61.6,55.5,49.1,44.9,43.9,43.6,36.5,35.7$, 35.5, 34.8, 34.6, 33.7, 29.7, 29.5, 29.0, 27.9, 27.3, 27.1, 26.7, 21.9, 21.4, 19.2, 17.0, 11.9, 11.7; HRMS (ESI) $\mathrm{C}_{62} \mathrm{H}_{76} \mathrm{O}_{11} \mathrm{SiNa}[\mathrm{M}+\mathrm{Na}]^{+}$calcd. 1047.5055, found 1047.5035.

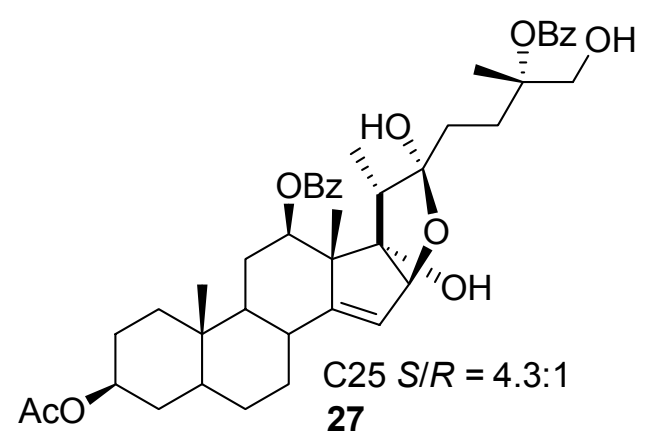

Hemiketal 27. TBAF, tetrabutylammonium fluoride, $1.0 \mathrm{M}$ solution in THF $(0.87 \mathrm{~mL}$, $0.87 \mathrm{mmol}, 2$ equiv) was added to a stirred solution of propionaldehyde $\mathbf{2 6}$ (446.5 $\mathrm{mg}$, $0.4355 \mathrm{mmol})$ in THF $(4.5 \mathrm{~mL})$ at $0{ }^{\circ} \mathrm{C}$. The reaction was stirred at $0{ }^{\circ} \mathrm{C}$ for $4 \mathrm{~h}$. On completion the reaction mixture was worked up with sat. $\mathrm{NH}_{4} \mathrm{Cl} / \mathrm{EtOAc}$, washed with brine, dried over $\mathrm{Na}_{2} \mathrm{SO}_{4}$. After removal of solvent under reduced pressure, the reaction mixture was purified by flash column chromatography (EtOAc/n-Hexane $=1: 2$ to $1: 1$ ) to provide hemiketal $27(270.5 \mathrm{mg}, 0.3701 \mathrm{mmol}, 85 \%)$ as white solid; mp $105 \sim 106{ }^{\circ} \mathrm{C}$; ${ }^{1} \mathrm{H}$ NMR $\left(300 \mathrm{MHz}, \mathrm{CDCl}_{3}\right) \delta 7.98-8.02(\mathrm{~m}, 4 \mathrm{H}), 7.41-7.53(\mathrm{~m}, 2 \mathrm{H}), 7.36-7.39(\mathrm{~m}$, $4 \mathrm{H}), 5.32(\mathrm{~s}, 1 \mathrm{H}), 5.21(\mathrm{dd}, J=11.1,4.8 \mathrm{~Hz}, 1 \mathrm{H}), 4.83(\mathrm{~s}, 1 \mathrm{H}), 4.60-4.68(\mathrm{~m}, 1 \mathrm{H}), 4.38$ (br s, 1H), $4.15-4.22$ (m, 2H), 3.34 (br s, 1H), 2.99 (s, 1H), 1.97 (s, 3H), 1.29 (s, 3H), $1.25(\mathrm{~s}, 3 \mathrm{H}), 0.99(\mathrm{~d}, J=6.7 \mathrm{~Hz}, 3 \mathrm{H}), 0.84(\mathrm{~s}, 3 \mathrm{H}) ;{ }^{13} \mathrm{C} \mathrm{NMR}\left(75 \mathrm{MHz}, \mathrm{CDCl}_{3}\right) \delta 170.5$, 166.4, 165.8, 153.5, 133.0, 132.7, 130.8, 129.8, 129.6, 129.4, 128.3, 128.2, 119.7, 108.0, 93.1, 91.4, 75.5, 73.2, 71.6, 71.3, 53.8, 52.1, 46.5, 44.1, 36.3, 35.9, 33.9, 33.6, 32.0, 31.0, 28.7, 27.8, 27.1, 23.6, 21.3, 14.9, 11.8, 7.8; HRMS (ESI) $\mathrm{C}_{43} \mathrm{H}_{52} \mathrm{O}_{9} \mathrm{Na}\left[\mathrm{M}-\mathrm{H}_{2} \mathrm{O}+\mathrm{Na}\right]^{+}$ calcd. 735.3509, found 735.3509. 


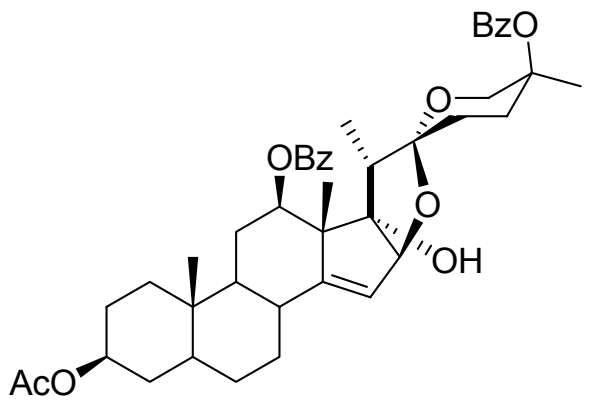

28

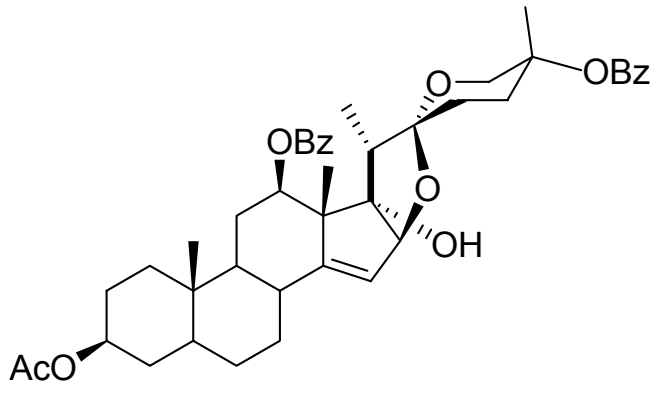

28-epi

28 (South 7) and 28-epi (C25-epi South 7). A solution of hemiketal $27(234.2 \mathrm{mg}$, $0.3204 \mathrm{mmol})$ and PPTS, pyridinium $p$-toluenesulfonate $(8.2 \mathrm{mg}, 0.0320 \mathrm{mmol}, 0.1$ equiv) in $\mathrm{CH}_{2} \mathrm{Cl}_{2}(3 \mathrm{~mL})$ was stirred at room temperature for $20 \mathrm{~min}$. After the reaction was completed, the reaction mixture was worked up with sat. $\mathrm{NaHCO}_{3} / \mathrm{EtOAc}$, washed with brine, dried over $\mathrm{Na}_{2} \mathrm{SO}_{4}$, and evaporated in vacuo. Flash column chromatography $($ EtOAc $/ \mathrm{n}-H e x a n e=1: 6$ to 1:4) provided compound 28, South $7(150.6 \mathrm{mg}, 0.2113 \mathrm{mmol}$, 66\%) and compound 28-epi, C25-epi South 7 (35.0 mg, $0.0491 \mathrm{mmol}, 15 \%)$ as white solid; mp compound $28129 \sim 130{ }^{\circ} \mathrm{C}\left(\mathrm{CH}_{2} \mathrm{Cl}_{2} / \mathrm{n}\right.$-Hexane), compound 28-epi $120 \sim 121$ ${ }^{\circ} \mathrm{C}\left(\mathrm{CH}_{2} \mathrm{Cl}_{2} / \mathrm{n}-\mathrm{Hexane}\right)$.

28 (South 7): ${ }^{1} \mathrm{H}$ NMR $\left(300 \mathrm{MHz}, \mathrm{CDCl}_{3}\right) \delta 8.01-8.06$ (m, 4H), $7.42-7.54(\mathrm{~m}, 2 \mathrm{H})$, $7.30-7.40(\mathrm{~m}, 4 \mathrm{H}), 5.28-5.31(\mathrm{~m}, 2 \mathrm{H}), 4.63-4.68(\mathrm{~m}, 2 \mathrm{H}), 4.30(\mathrm{~d}, J=11.1 \mathrm{~Hz}, 1 \mathrm{H})$, $4.16(\mathrm{~d}, J=11.1 \mathrm{~Hz}, 1 \mathrm{H}), 2.57$ (s, 1H), $2.02-2.29$ (m, 2H), 1.97 (s, 3H), $1.32(\mathrm{~s}, 3 \mathrm{H})$, $1.16(\mathrm{~s}, 3 \mathrm{H}), 0.96(\mathrm{~d}, J=6.7 \mathrm{~Hz}, 3 \mathrm{H}), 0.84(\mathrm{~s}, 3 \mathrm{H}) ;{ }^{13} \mathrm{C} \mathrm{NMR}\left(75 \mathrm{MHz}, \mathrm{CDCl}_{3}\right) \delta 170.4$, 166.2, 165.5, 154.6, 132.8, 132.4, 131.2, 130.1, 129.6, 129.4, 128.2, 128.1, 119.0, 117.7, 93.6, 90.3, 83.0, 74.9, 73.1, 69.9, 53.6, 51.8, 44.3, 44.0, 36.3, 35.8, 33.9, 33.6, 32.5, 31.8, 28.8, 27.9, 27.1, 23.8, 21.2, 15.4, 11.8, 7.6; HRMS (ESI) $\mathrm{C}_{43} \mathrm{H}_{52} \mathrm{O}_{9} \mathrm{Na}[\mathrm{M}+\mathrm{Na}]^{+}$calcd. 735.3509 , found 735.3504 .

28-epi (C25-epi South 7): ${ }^{1} \mathrm{H}$ NMR (300 MHz, $\left.\mathrm{CDCl}_{3}\right) \delta 8.00(\mathrm{~d}, J=7.3 \mathrm{~Hz}, 2 \mathrm{H}), 7.94(\mathrm{~d}$, $J=7.5 \mathrm{~Hz}, 2 \mathrm{H}), 7.48-7.53$ (m, 2H), $7.33-7.41$ (m, 4H), 5.39 (s, 1H), 5.31 (dd, $J=11.2$, $5.0 \mathrm{~Hz}, 1 \mathrm{H}), 4.74(\mathrm{~s}, 1 \mathrm{H}), 4.65-4.70(\mathrm{~m}, 1 \mathrm{H}), 4.11(\mathrm{~d}, J=11.3 \mathrm{~Hz}, 1 \mathrm{H}), 4.00(\mathrm{~d}, J=$ $11.3 \mathrm{~Hz}, 1 \mathrm{H}), 2.68$ (s, 1H), $2.02-2.18$ (m, 5H), 1.99 (s, 3H), 1.38 (s, 3H), 1.31 (s, 3H), $0.92(\mathrm{~d}, J=6.6 \mathrm{~Hz}, 3 \mathrm{H}), 0.86(\mathrm{~s}, 3 \mathrm{H}) ;{ }^{13} \mathrm{C} \mathrm{NMR}\left(75 \mathrm{MHz}, \mathrm{CDCl}_{3}\right) \delta 170.5,166.3,165.8$, 154.5, 133.0, 132.5, 131.3, 129.9, 129.51, 129.48, 128.3, 128.2, 119.4, 117.7, 93.4, 90.7, 
82.9, 75.2, 73.3, 69.7, 53.7, 52.1, 44.4, 44.2, 36.4, 35.9, 34.0, 33.7, 33.0, 32.9, 28.9, 28.0, 27.3, 27.2, 25.9, 21.4, 15.2, 11.9, 7.6; HRMS (ESI) $\mathrm{C}_{43} \mathrm{H}_{52} \mathrm{O}_{9} \mathrm{Na}[\mathrm{M}+\mathrm{Na}]^{+}$calcd. 735.3509 , found 735.3515 .

\section{Discussion on the facial selectivity of $[4+2]$ cycloaddition}

The facial selectivity of [4+2] cycloaddition is originated from the conformational effect of $\mathrm{C} 21-\mathrm{Me}$ and sterically demanding 1,3-dioxane group. In the case of C21- $\alpha-\mathrm{Me}$, methyl group is directing to $\alpha$-face having 1,3-dioxane group block $\beta$-face of cyclopentadiene. For this reason, only $\alpha$-face is available to react with singlet oxygen. In contrast, C21- $\beta$ $\mathrm{Me}$ is directing to $\beta$-face forcing $\alpha$-face blocked by 1,3-dioxane group. Molecular mechanics reveals the C-26 trifluoroacetate precursors of C-22 ketals 16f- $\alpha$ (left) and 16d- $\beta$ (right), predicts the C-21 methyl stereocenter will control the sidechain conformation. The $4-5$ lowest energy conformations $( \pm 2.5 \mathrm{kcal})$ are superimposed in the graphics.

Figure 1.

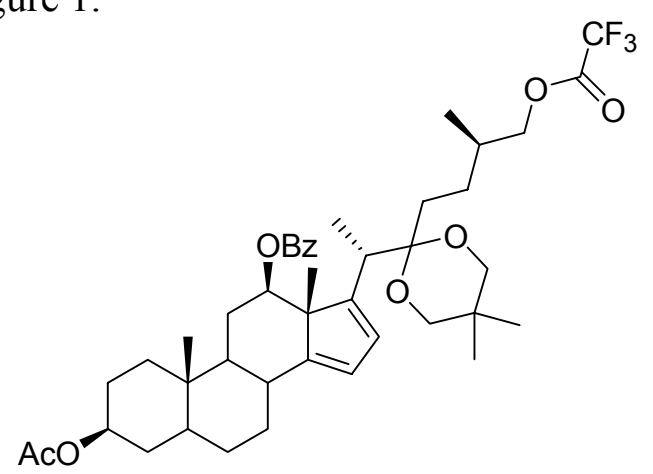

16f C21- $\alpha-\mathrm{Me}$

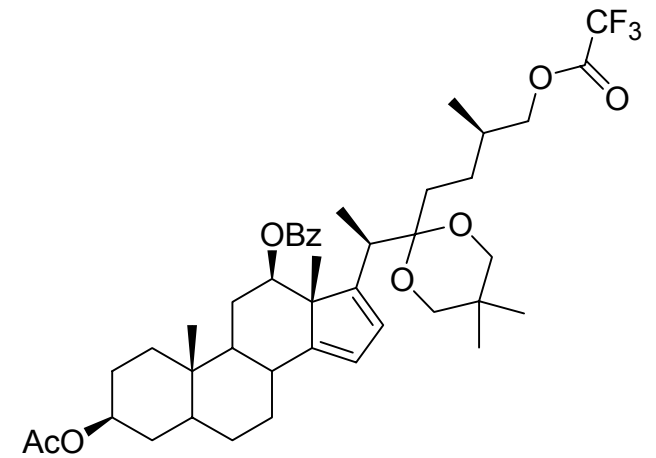

16d C21- $\beta-M e$

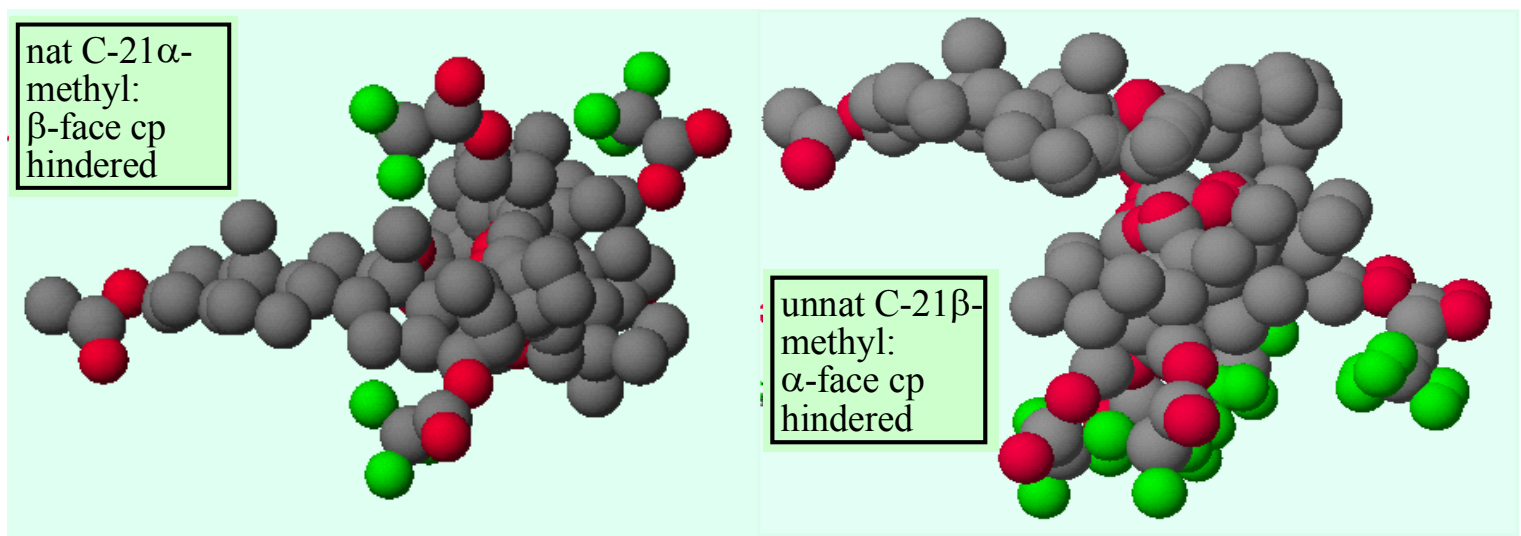


To experimentally prove this prediction, diol 35,36 were prepared through different synthetic routes (Scheme 1). For preparation of diol 35 (C21- $\alpha-\mathrm{Me})$, dienyl ketone 15c was protected to 5,5-dimethyl-1,3-dioxane, treated with singlet oxygen, and reduced with activated Zn. Diol $36(\mathrm{C} 21-\beta-\mathrm{Me})$ was prepared in 7 steps from diene 13. Comparison of NMR chemical shifts (see spectrum section) revealed the facial selectivity of [4+2] cycloaddition reaction of singlet oxygen is in excellent control. Additionally, x-ray structure and NMR spectrum of endoperoxide 20a- $\alpha$ are available.

Scheme 1.
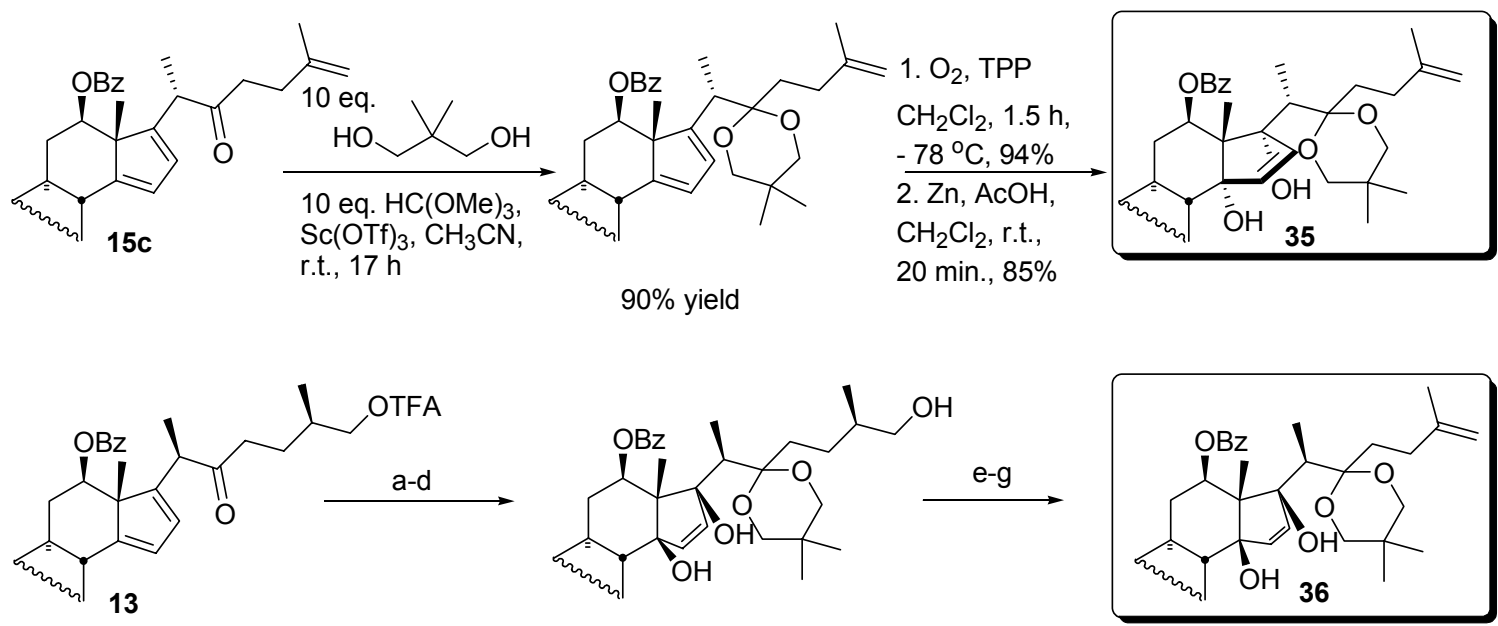

(a) TMSOCH $\mathrm{C}_{2}\left(\mathrm{CH}_{3}\right)_{2} \mathrm{CH}_{2} \mathrm{OTMS}$, cat. TMSOTf, $\mathrm{CH}_{2} \mathrm{Cl}_{2}, 79 \%$; (b) TPP, $\mathrm{O}_{2}, \mathrm{CH}_{2} \mathrm{Cl}_{2}, 81 \%$; (c) $\mathrm{Zn}, \mathrm{AcOH}, \mathrm{CH}_{2} \mathrm{Cl}_{2}$, 91\%; (d) $\mathrm{Na}_{2} \mathrm{CO}_{3}$, THF, $\mathrm{H}_{2} \mathrm{O}, 94 \%$; (e) TsCl, TEA, DMAP, $\mathrm{CH}_{2} \mathrm{Cl}_{2}, 93 \%$; (f) Nal, pyridine, $\mathrm{CH}_{3} \mathrm{CN}$; (g) DBU, DMF, heating, $73 \%$ over two steps.

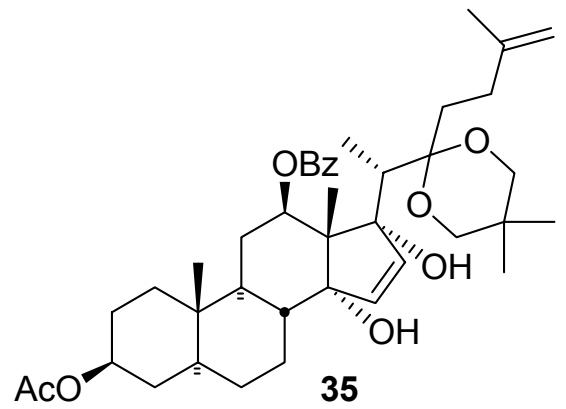

White solid; mp $125 \sim 127{ }^{\circ} \mathrm{C} ;{ }^{1} \mathrm{H}$ NMR $\left(300 \mathrm{MHz}, \mathrm{CDCl}_{3}\right) \delta 7.99(\mathrm{~d}, J=7.6 \mathrm{~Hz}, 2 \mathrm{H})$, $7.45-7.50$ (m, 1H), $7.34-7.39$ (m, 2H), 6.99 (d, $J=6.2 \mathrm{~Hz}, 1 \mathrm{H}), 6.16$ (d, $J=6.3 \mathrm{~Hz}$, 1H), 6.09 (dd, $J=10.1,5.6 \mathrm{~Hz}, 1 \mathrm{H}), 4.78(\mathrm{~s}, 1 \mathrm{H}), 4.61-4.63(\mathrm{~m}, 3 \mathrm{H}), 3.81(\mathrm{~s}, 1 \mathrm{H}), 3.53$ (dd, $J=11.5,5.7 \mathrm{~Hz}, 2 \mathrm{H}), 3.33$ (dd, $J=23.0,11.6 \mathrm{~Hz}, 2 \mathrm{H}), 2.14-2.27$ (m, 2H), 1.93 (s, $3 \mathrm{H}), 1.66$ (s, 3H), 1.08 (s, 3H), 1.00 (s, 3H), 0.93 (d, $J=7.0 \mathrm{~Hz}, 3 \mathrm{H}), 0.83$ (s, 3H), 0.78 (s, $3 \mathrm{H}) ;{ }^{13} \mathrm{C}$ NMR (75 MHz, $\left.\mathrm{CDCl}_{3}\right) \delta 170.2,165.3,145.2,140.6,135.4,132.5,130.9,129.4$, 
$128.1,109.6,104.5,90.2,87.9,73.3,71.8,69.5,69.3,56.5,45.3,44.1,37.8,36.1,35.5$, 34.6, 34.5, 33.7, 30.8, 29.1, 28.3, 27.5, 27.3, 27.1, 26.1, 23.7, 22.6, 21.2, 14.8, 11.4, 10.4; HRMS (ESI) $\mathrm{C}_{41} \mathrm{H}_{58} \mathrm{O}_{8} \mathrm{Na}[\mathrm{M}+\mathrm{Na}]^{+}$calcd. 701.4029, found 701.4026.

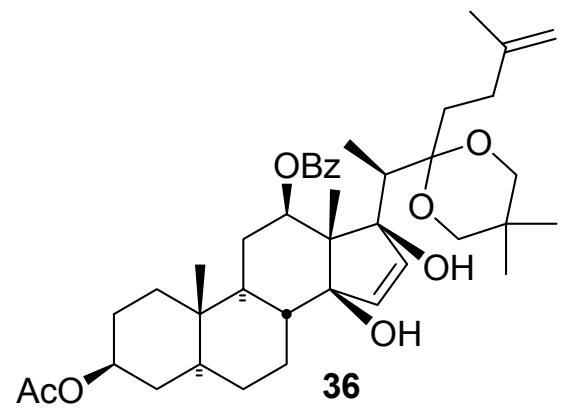

White solid; mp $130 \sim 132{ }^{\circ} \mathrm{C} ;{ }^{1} \mathrm{H}$ NMR $\left(300 \mathrm{MHz}, \mathrm{CDCl}_{3}\right) \delta 8.02(\mathrm{~d}, J=7.2 \mathrm{~Hz}, 2 \mathrm{H})$, $7.49-7.54(\mathrm{~m}, 1 \mathrm{H}), 7.38-7.43(\mathrm{~m}, 2 \mathrm{H}), 7.02$ (d, $J=6.2 \mathrm{~Hz}, 1 \mathrm{H}), 6.19$ (d, $J=6.3 \mathrm{~Hz}$, $1 \mathrm{H}), 6.12(\mathrm{dd}, J=10.2,5.6 \mathrm{~Hz}, 1 \mathrm{H}), 4.84(\mathrm{~s}, 1 \mathrm{H}), 4.64-4.67(\mathrm{~m}, 3 \mathrm{H}), 3.83(\mathrm{~s}, 1 \mathrm{H}), 3.58$ (dd, $J=11.6,4.8 \mathrm{~Hz}, 2 \mathrm{H}), 3.36$ (dd, $J=24.7,12.0 \mathrm{~Hz}, 2 \mathrm{H}), 2.14-2.32$ (m, 2H), 1.98 (s, 3H), 1.69 (s, 3H), 1.09 (s, 3H), 1.05 (s, 3H), 0.96 (d, J=7.2 Hz, 3H), 0.87 (s, 3H), 0.80 (s, $3 \mathrm{H}) ;{ }^{13} \mathrm{C} \mathrm{NMR}\left(75 \mathrm{MHz}, \mathrm{CDCl}_{3}\right) \delta 170.4,165.5,145.4,140.7,135.7,132.6,131.0,129.6$, $128.2,109.7,104.9,90.5,88.0,73.5,71.9,69.7,69.5,56.6,45.4,44.2,38.4,36.3,35.7$, $34.8,33.8,31.0,29.3,28.5,27.5,27.2,26.3,23.9,22.8,22.7,21.4,14.9,11.5,10.6$; HRMS (ESI) $\mathrm{C}_{41} \mathrm{H}_{58} \mathrm{O}_{8} \mathrm{Na}[\mathrm{M}+\mathrm{Na}]^{+}$calcd. 701.4029, found 701.4031. 


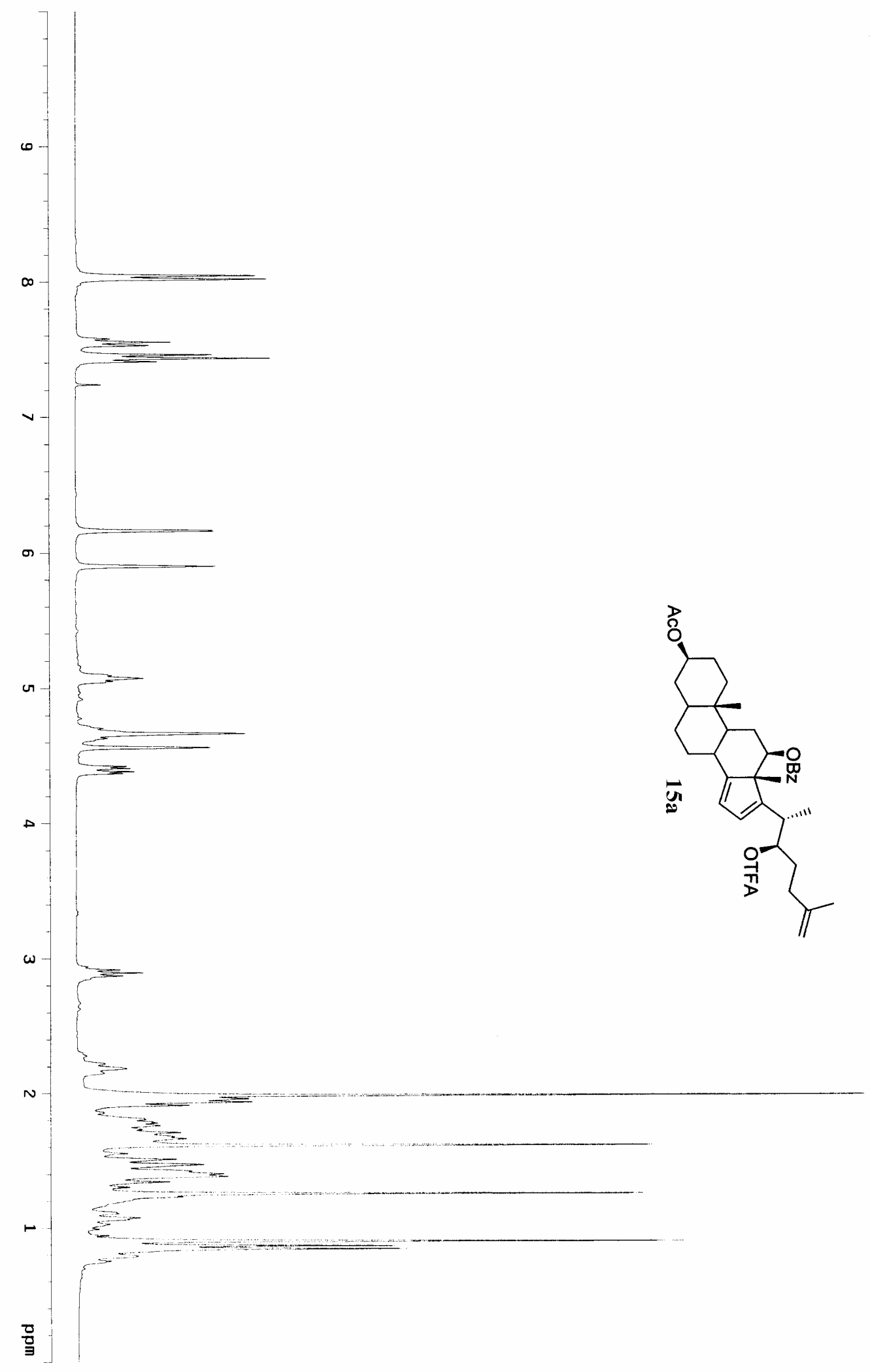



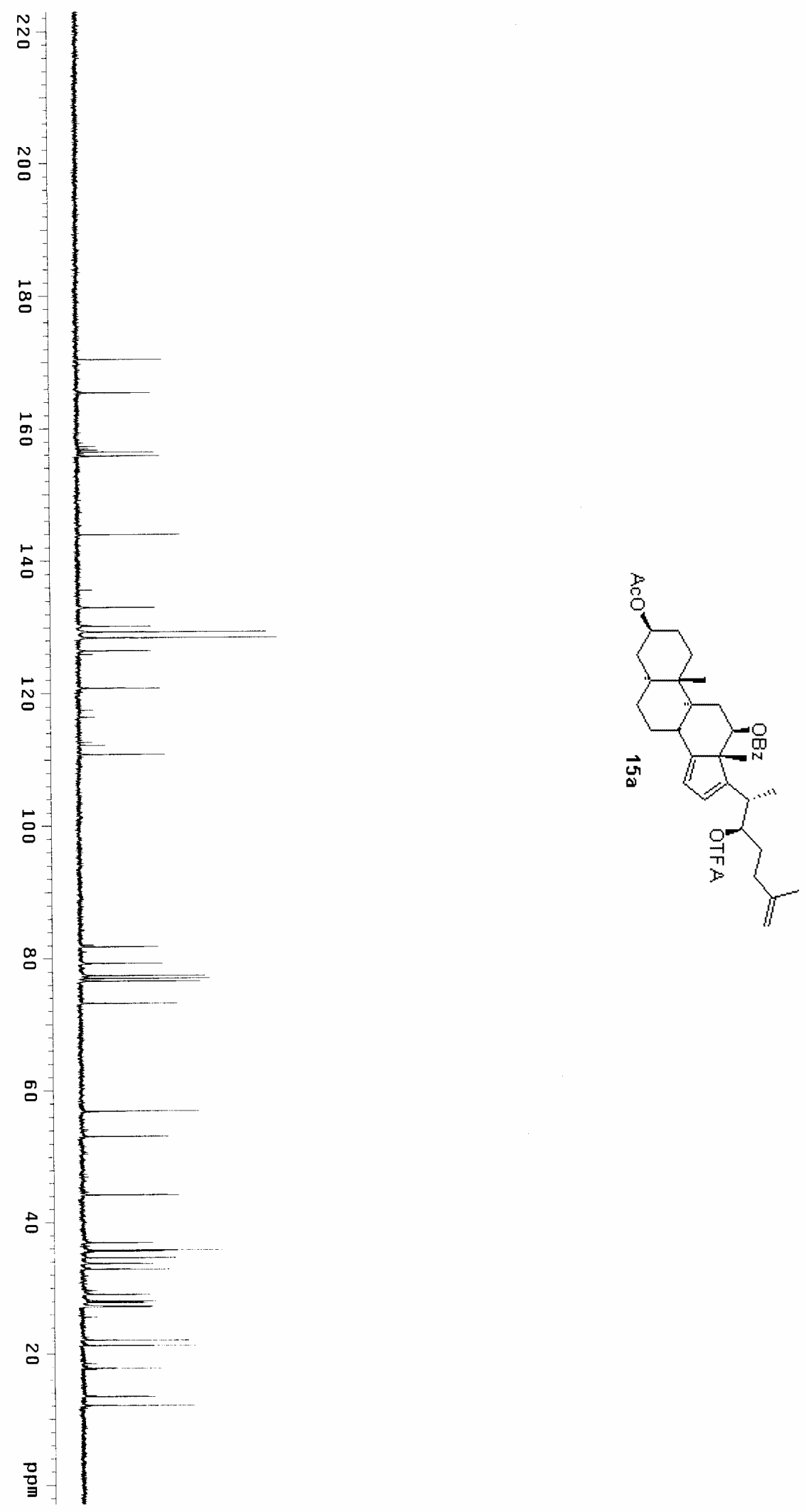


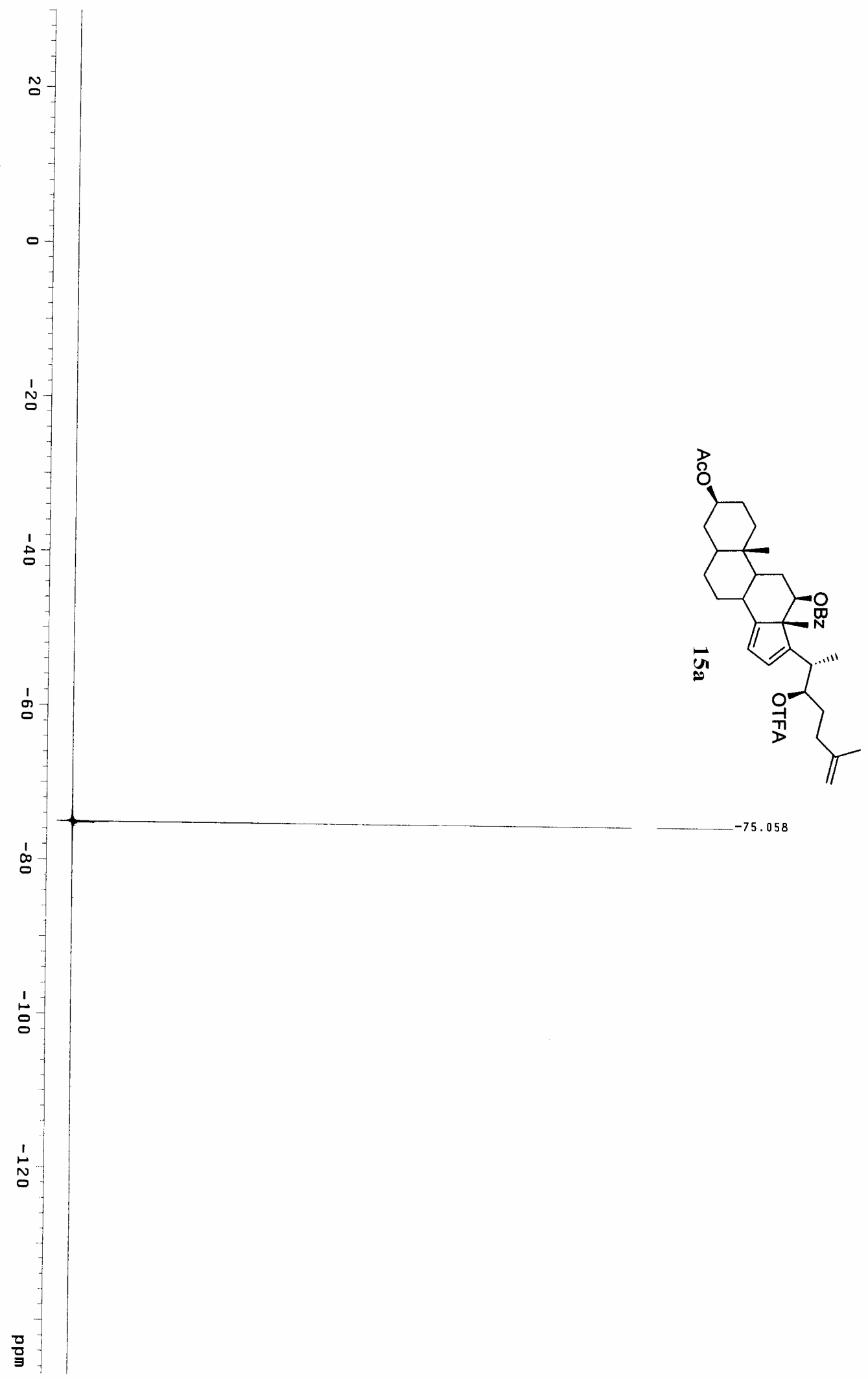

S- 31 


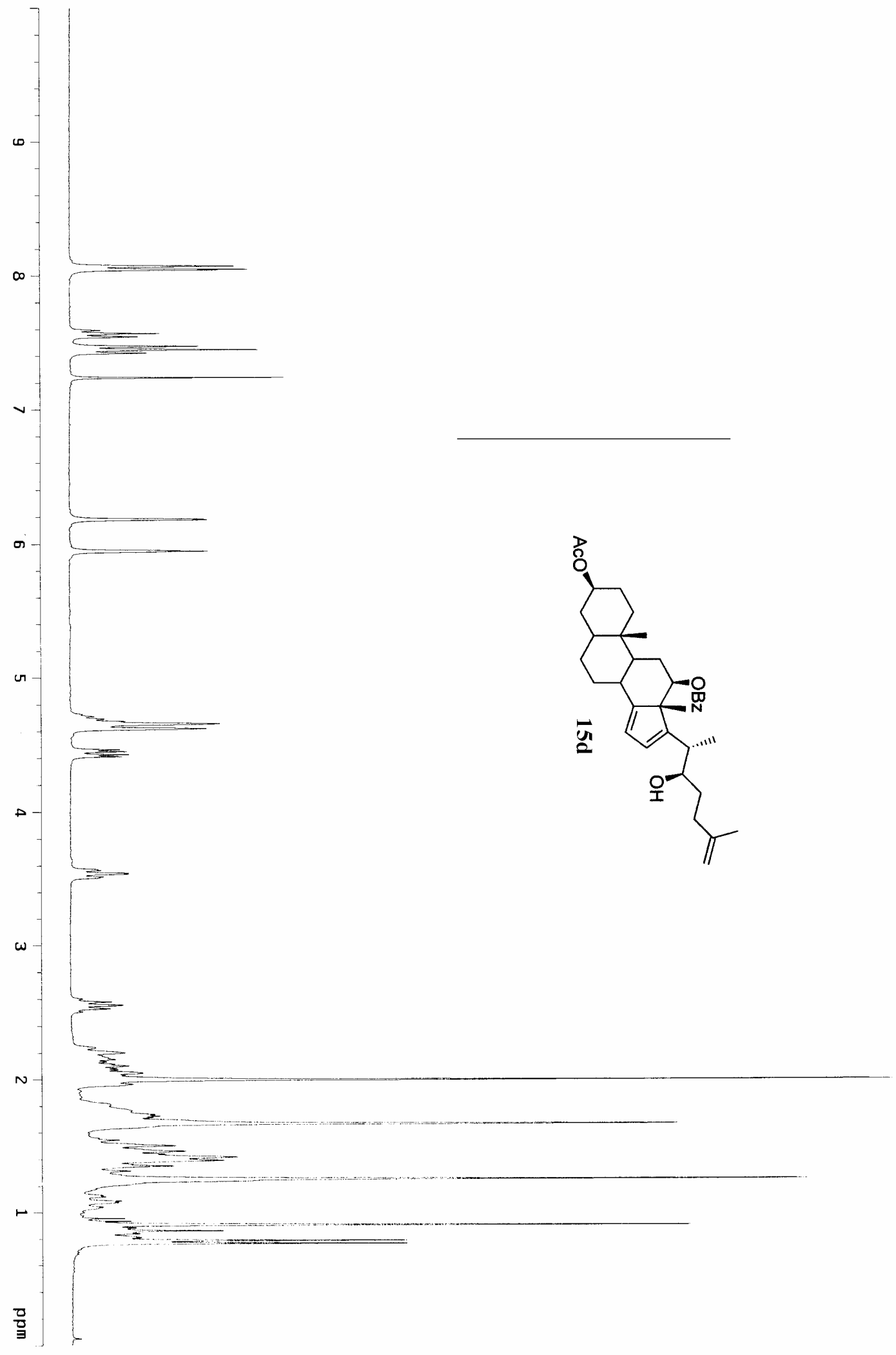

S- 32 

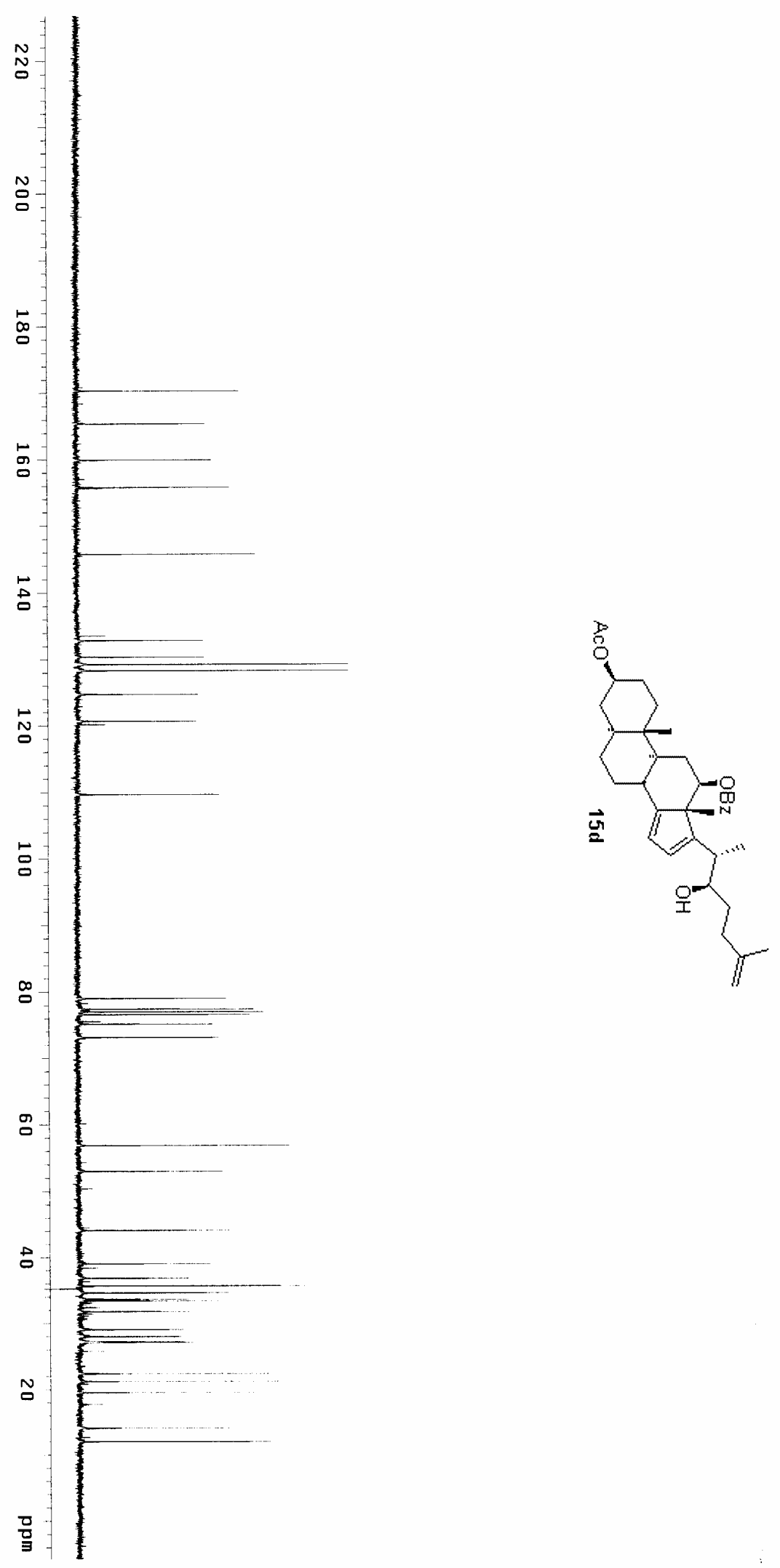


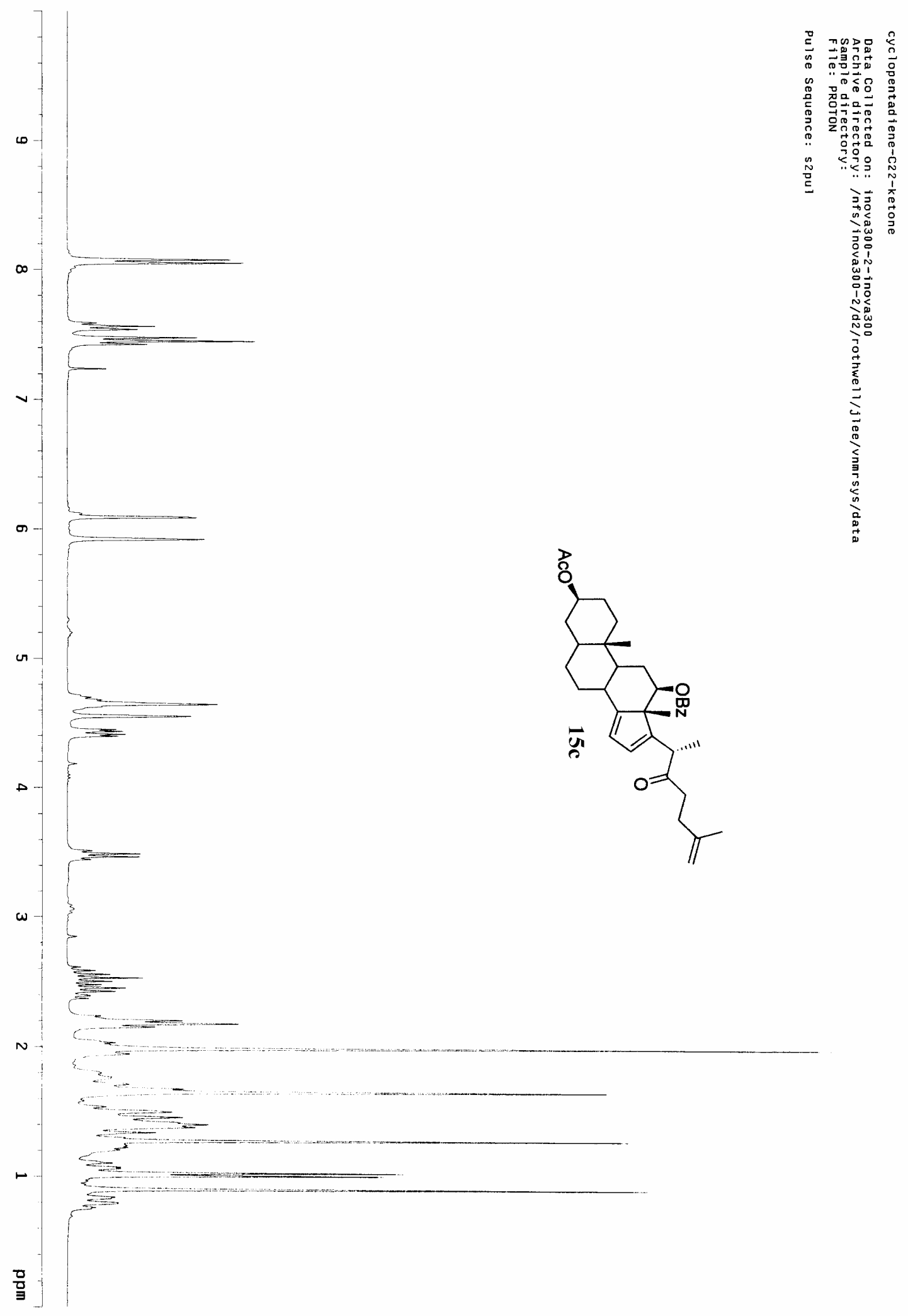



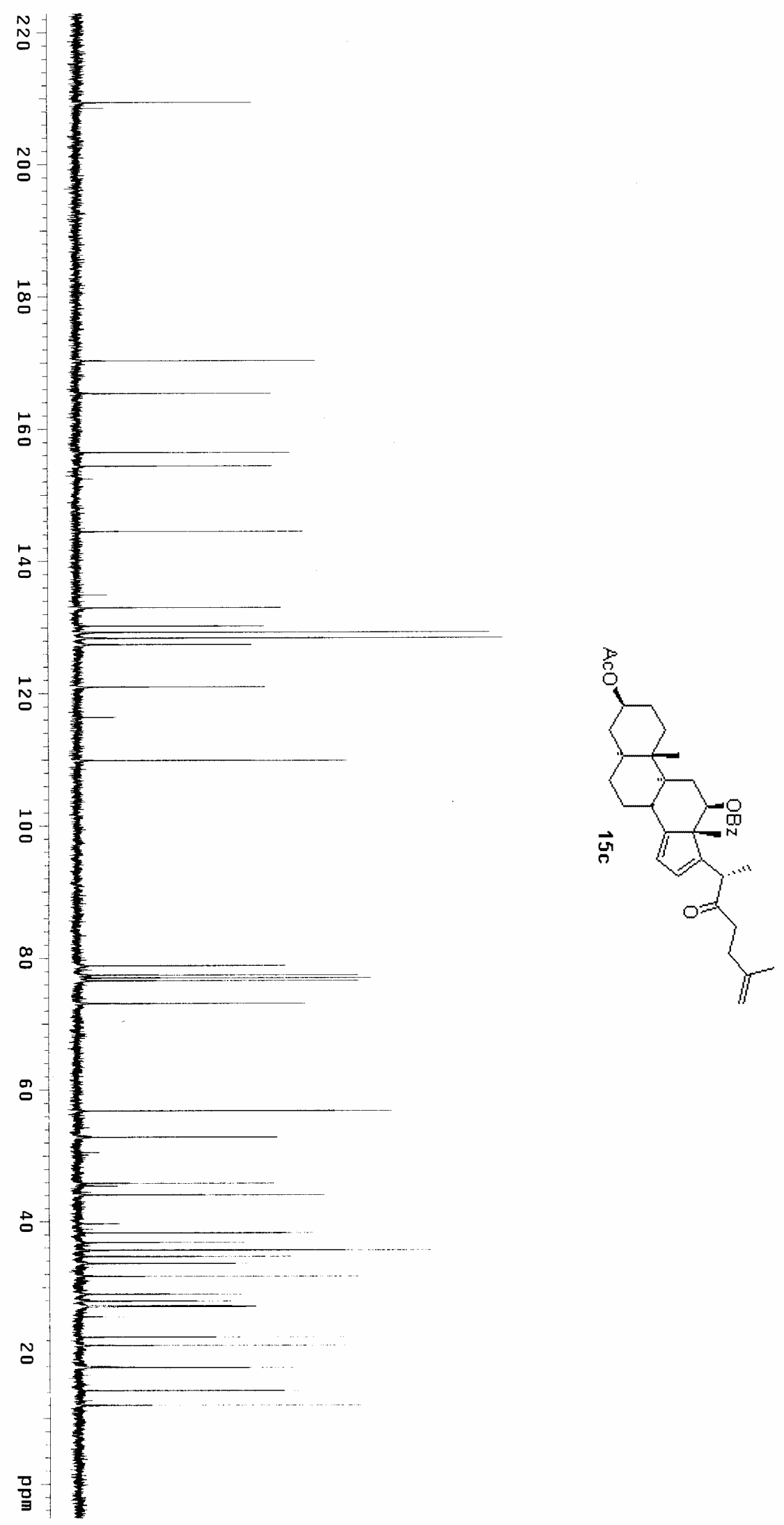


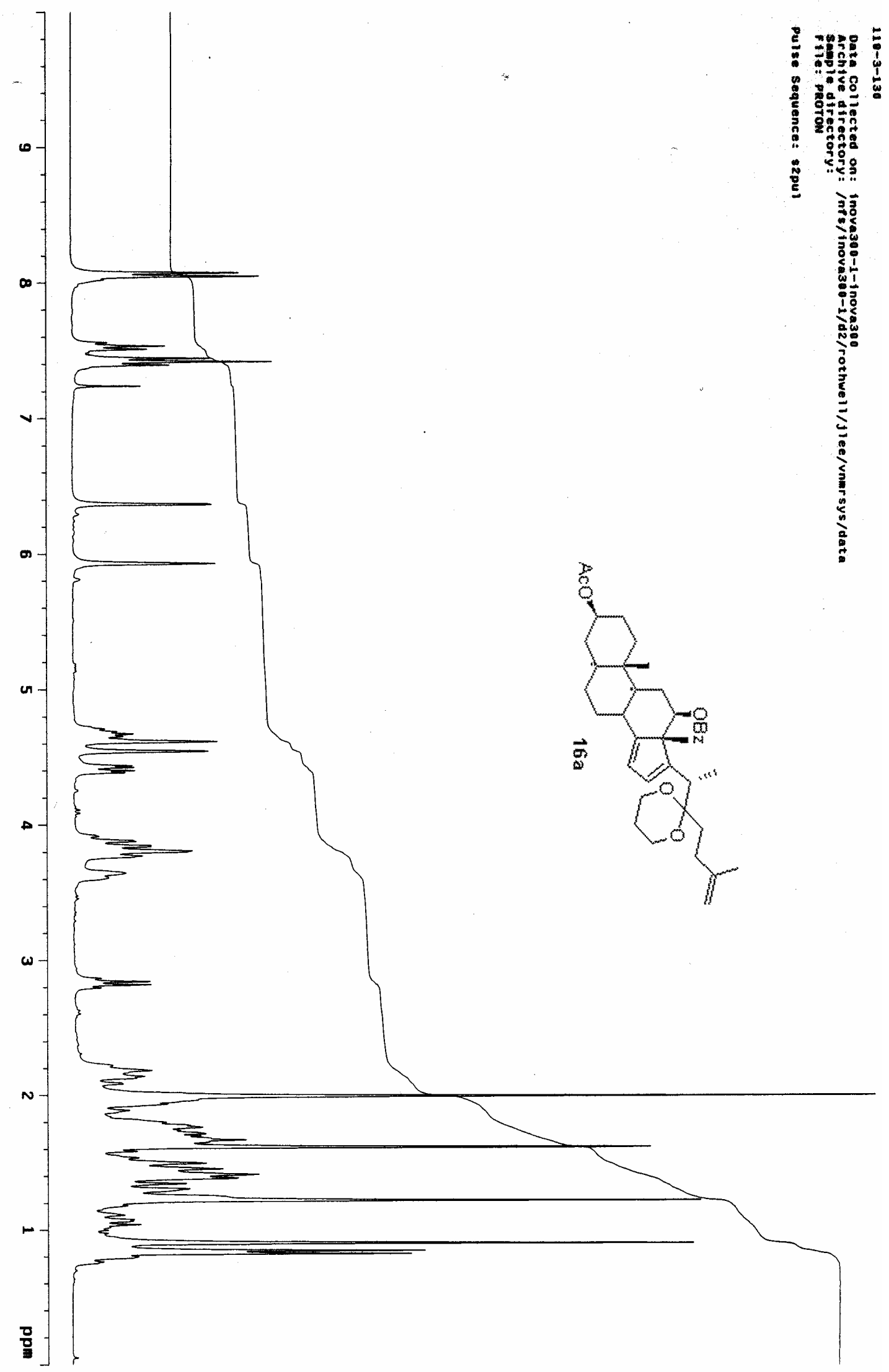

S- 36 

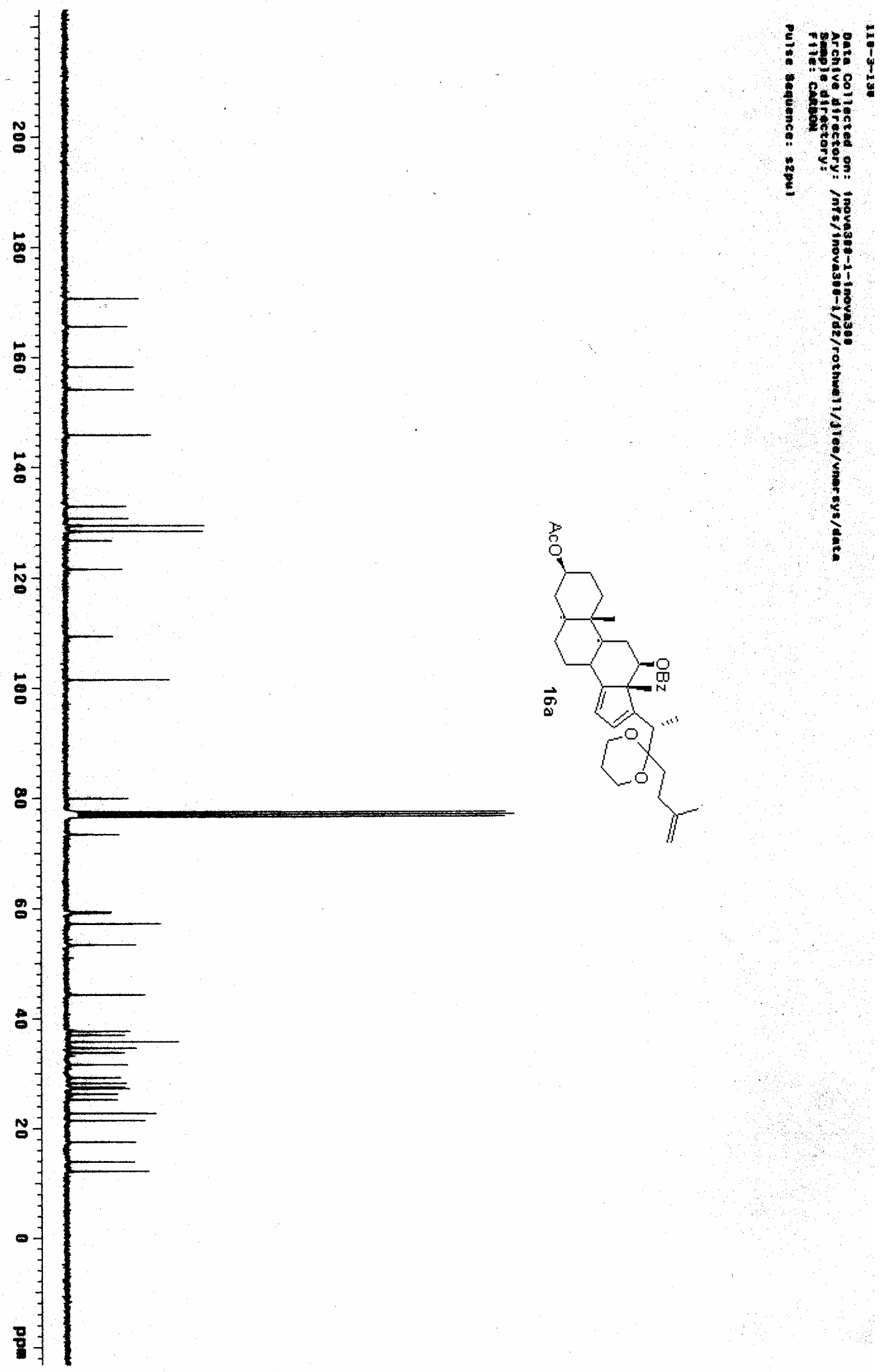


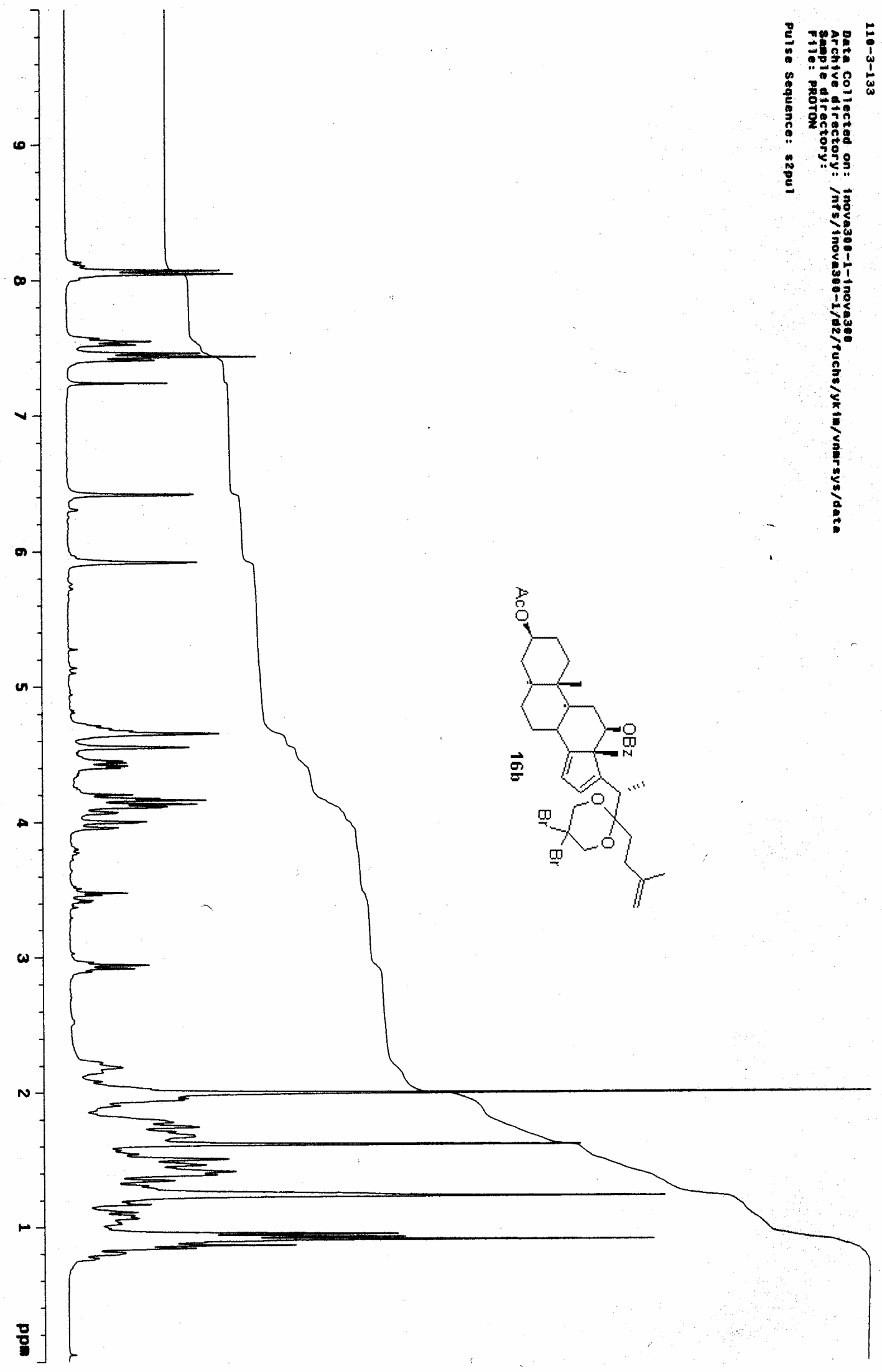

S- 38 


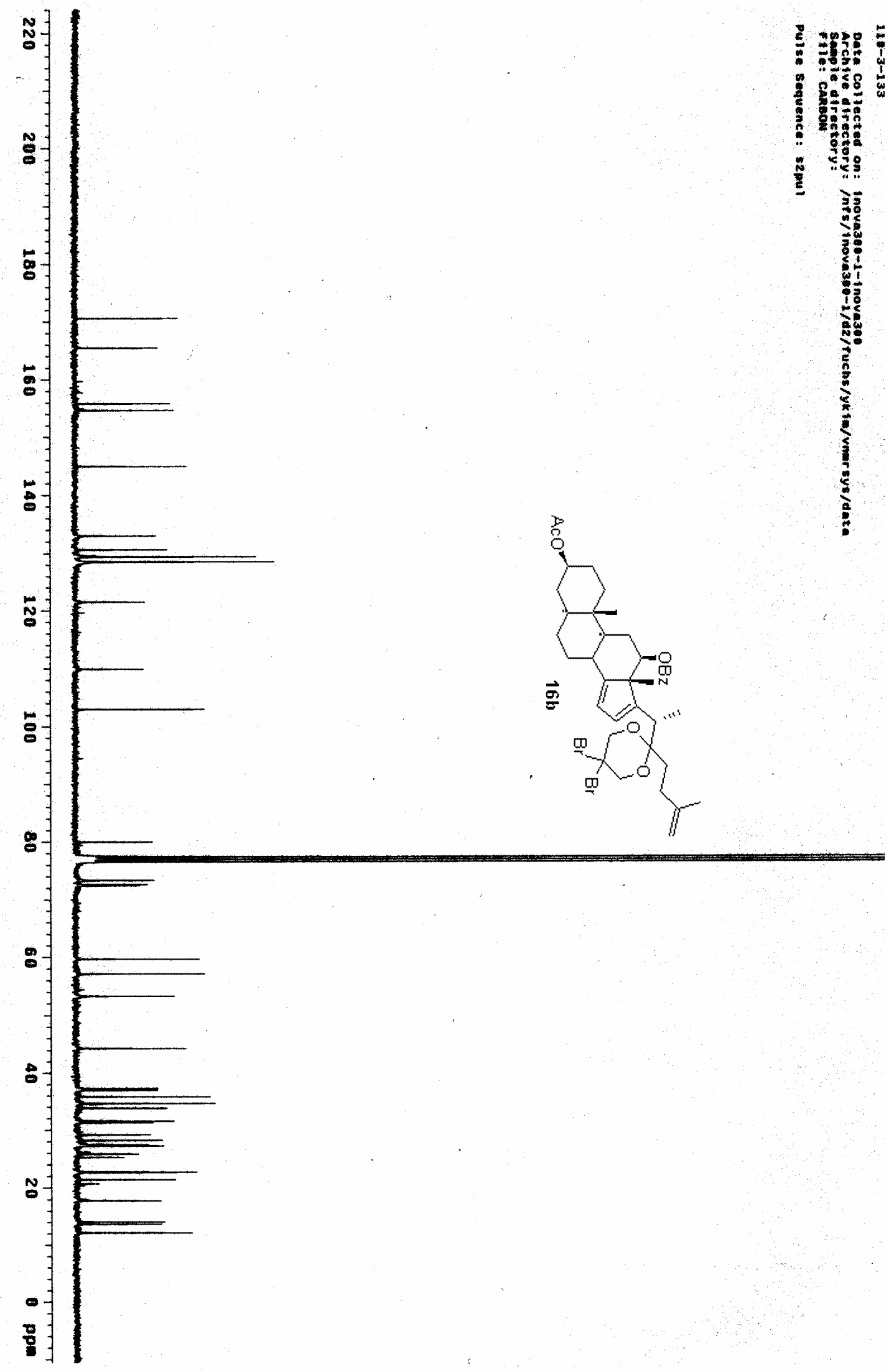




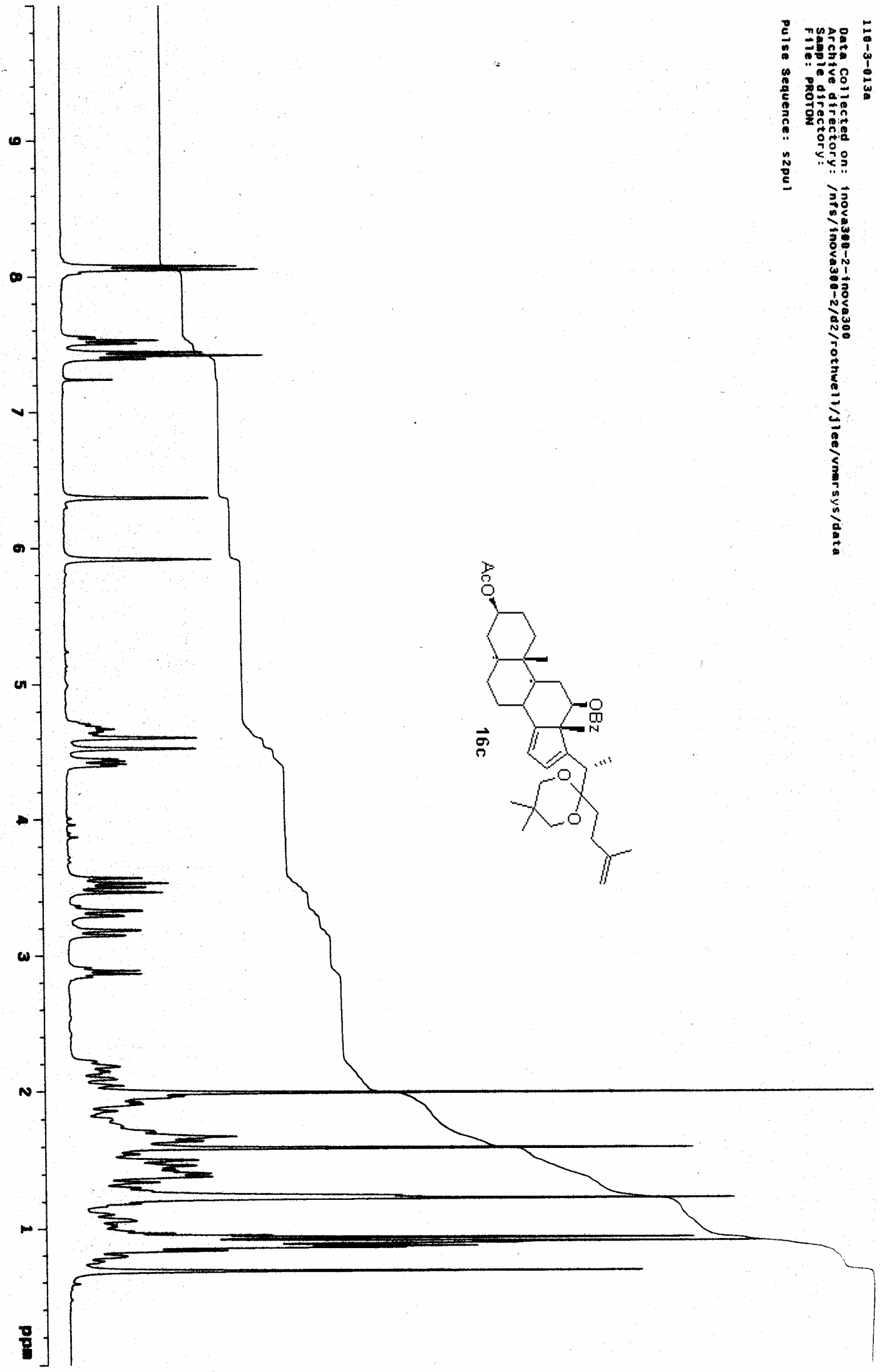

S- 40 

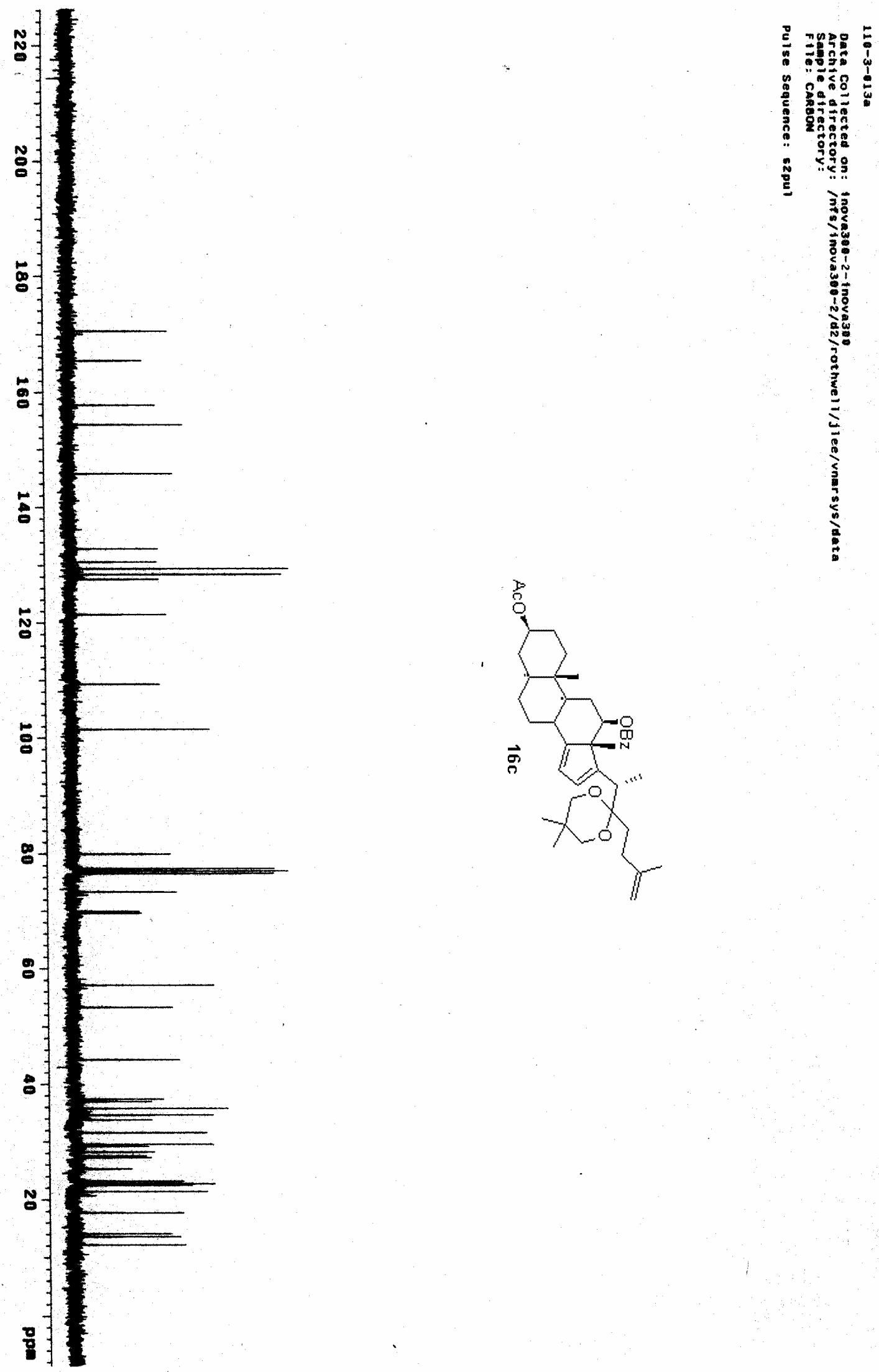


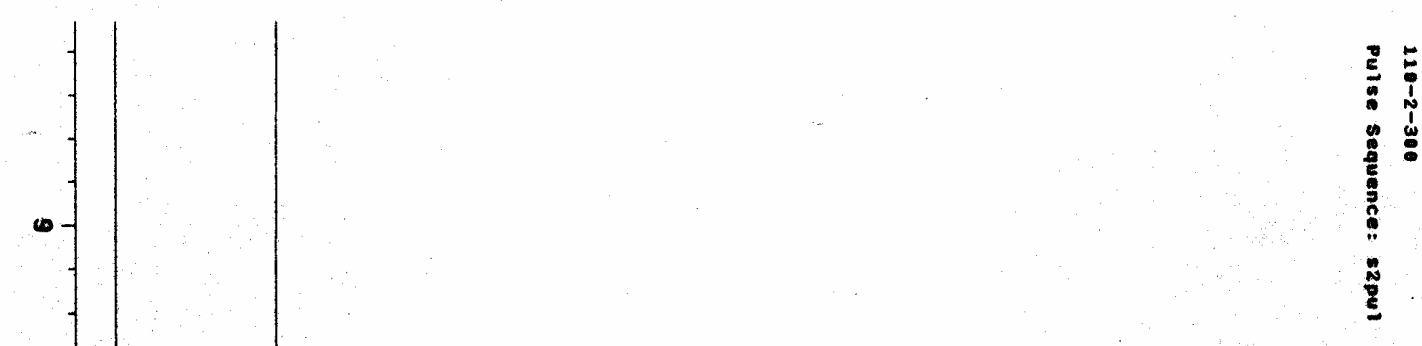

. 

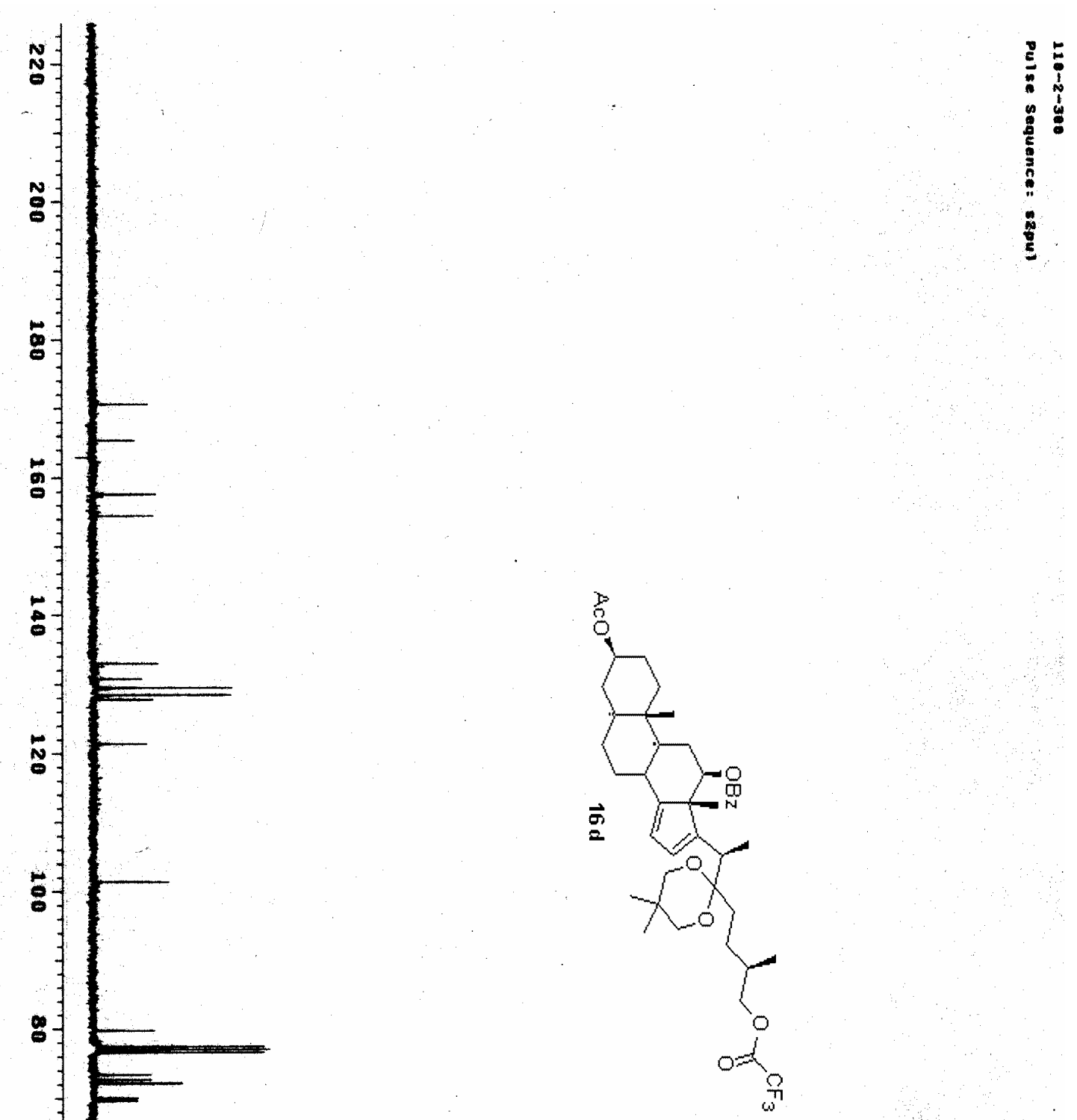


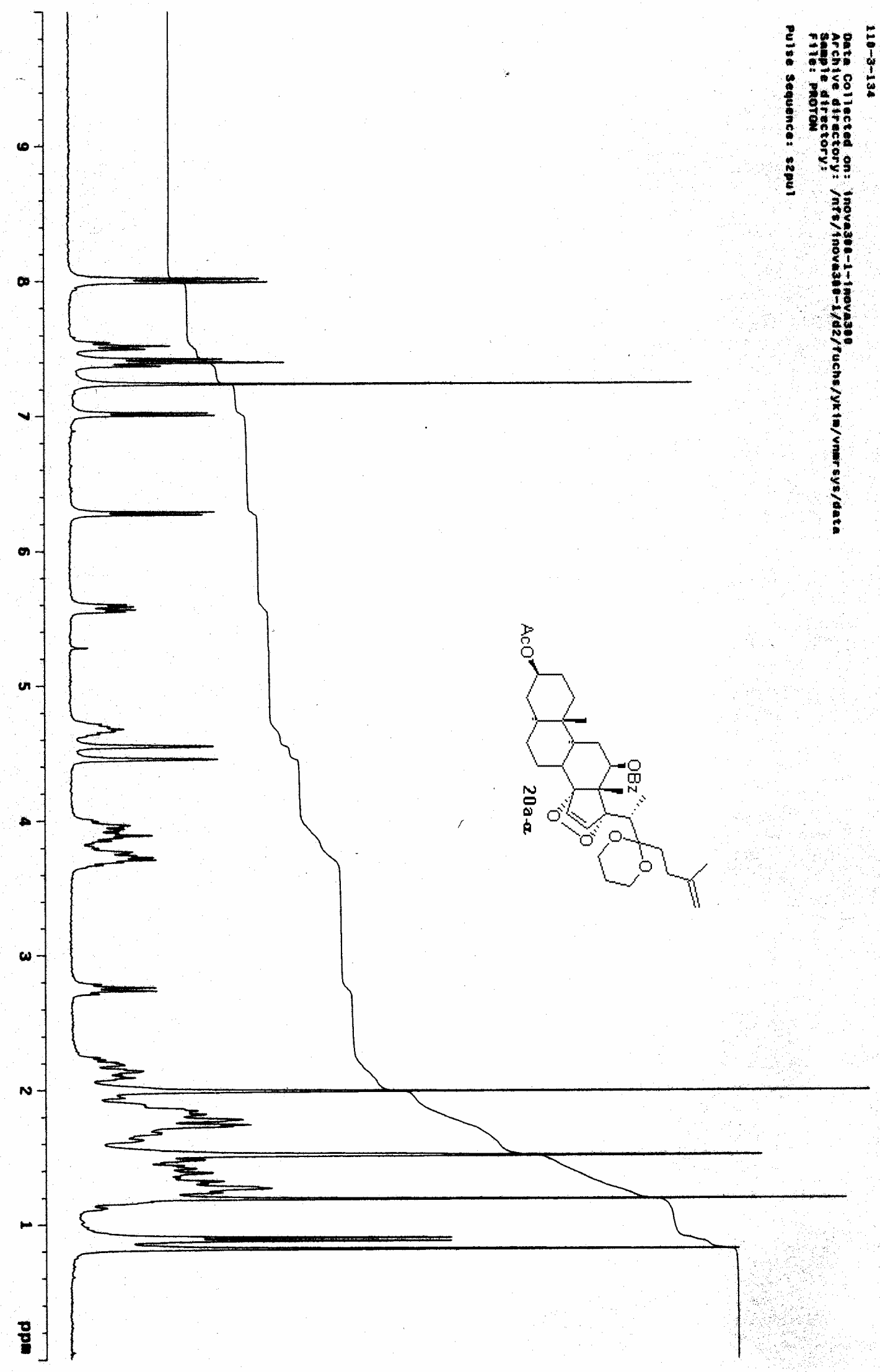




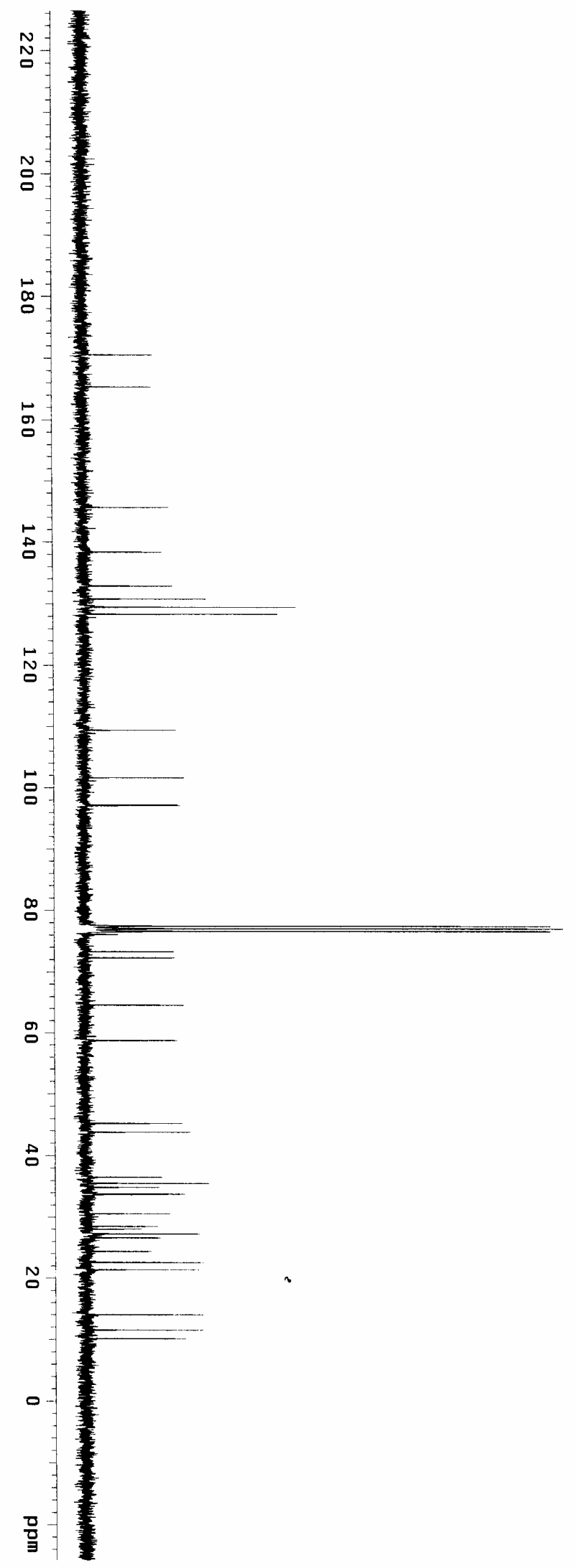

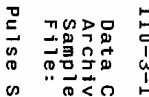

का

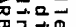

2

赵

دे

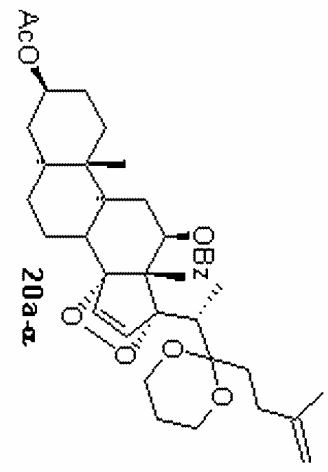




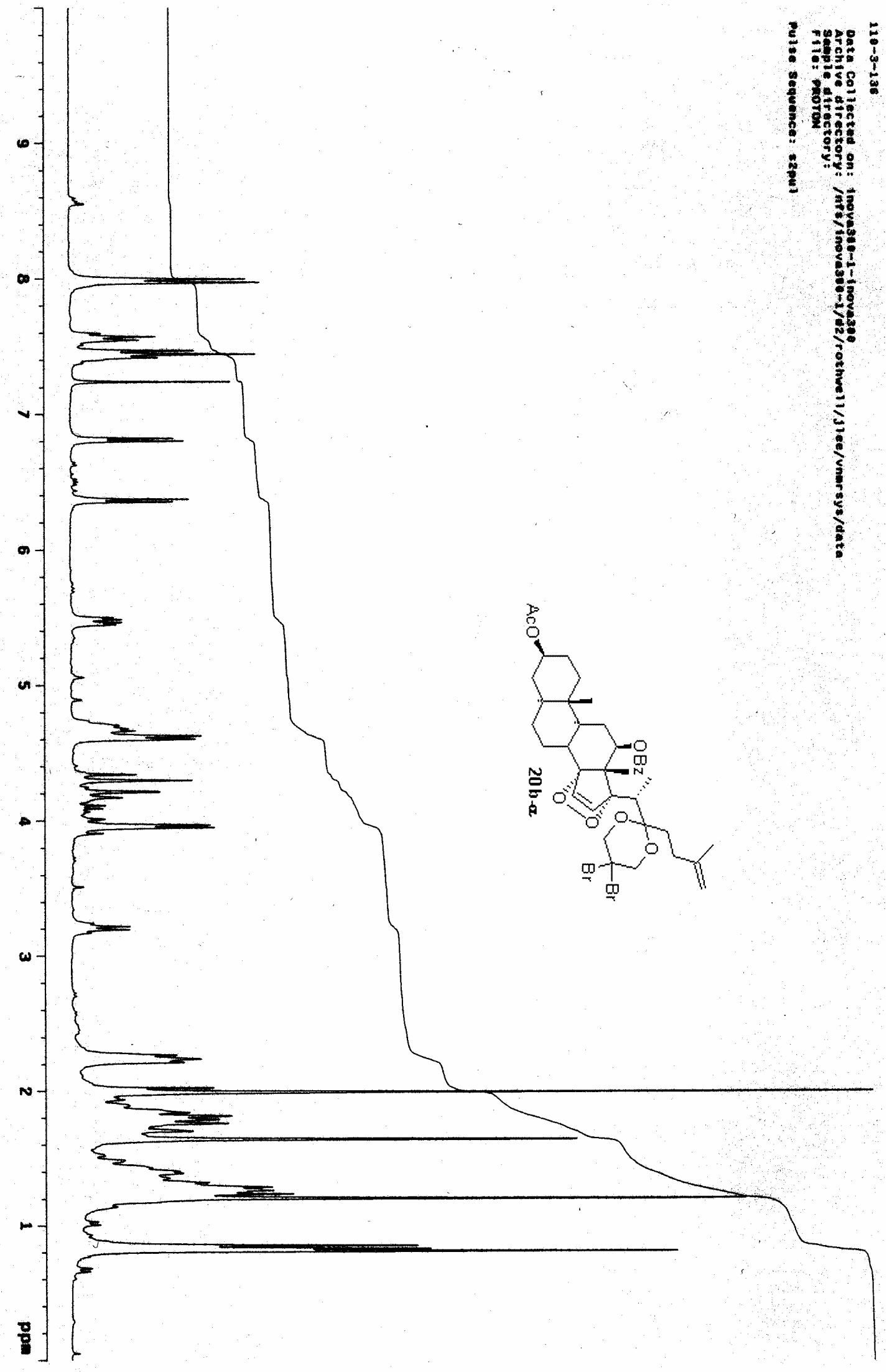




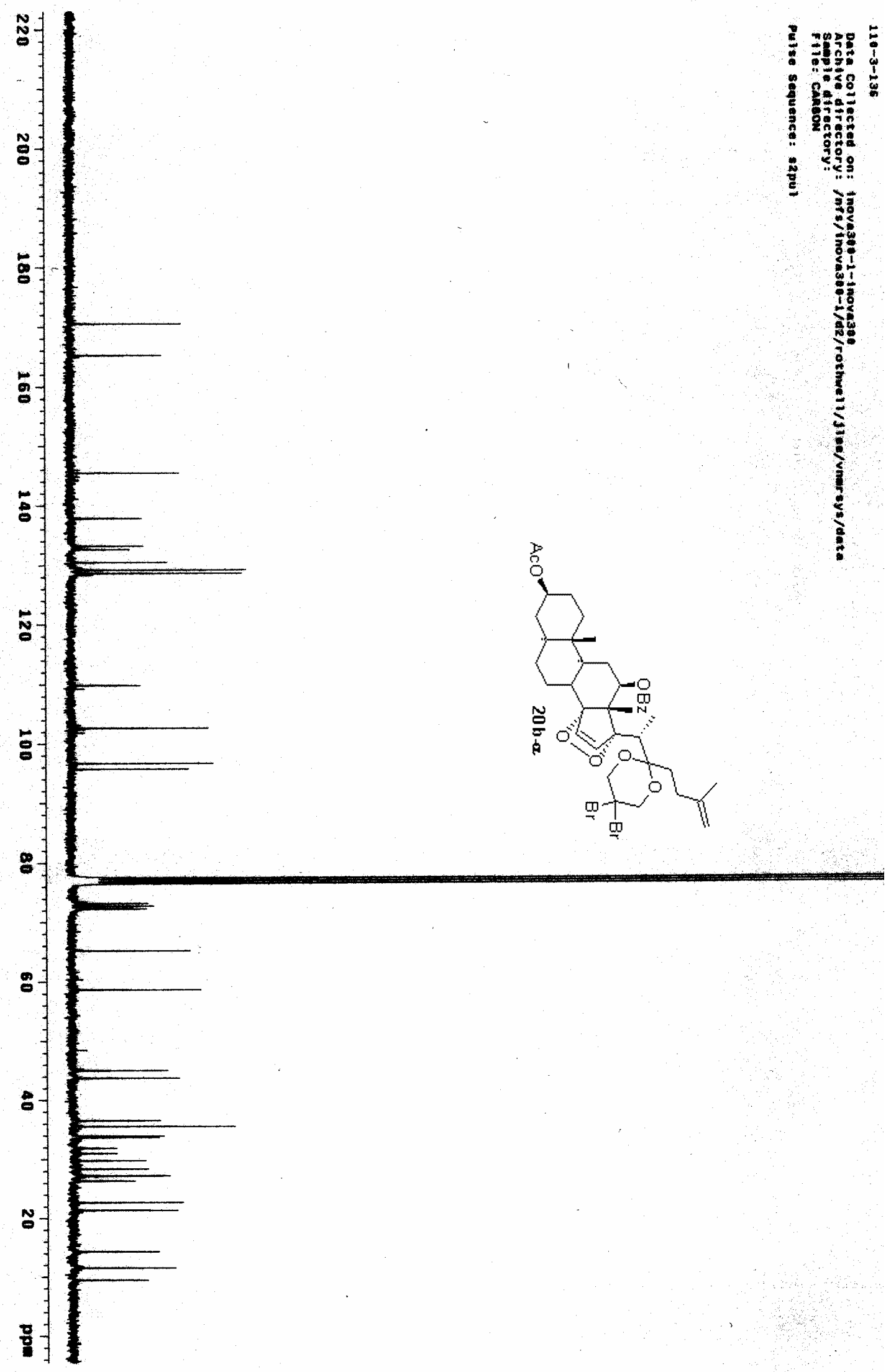




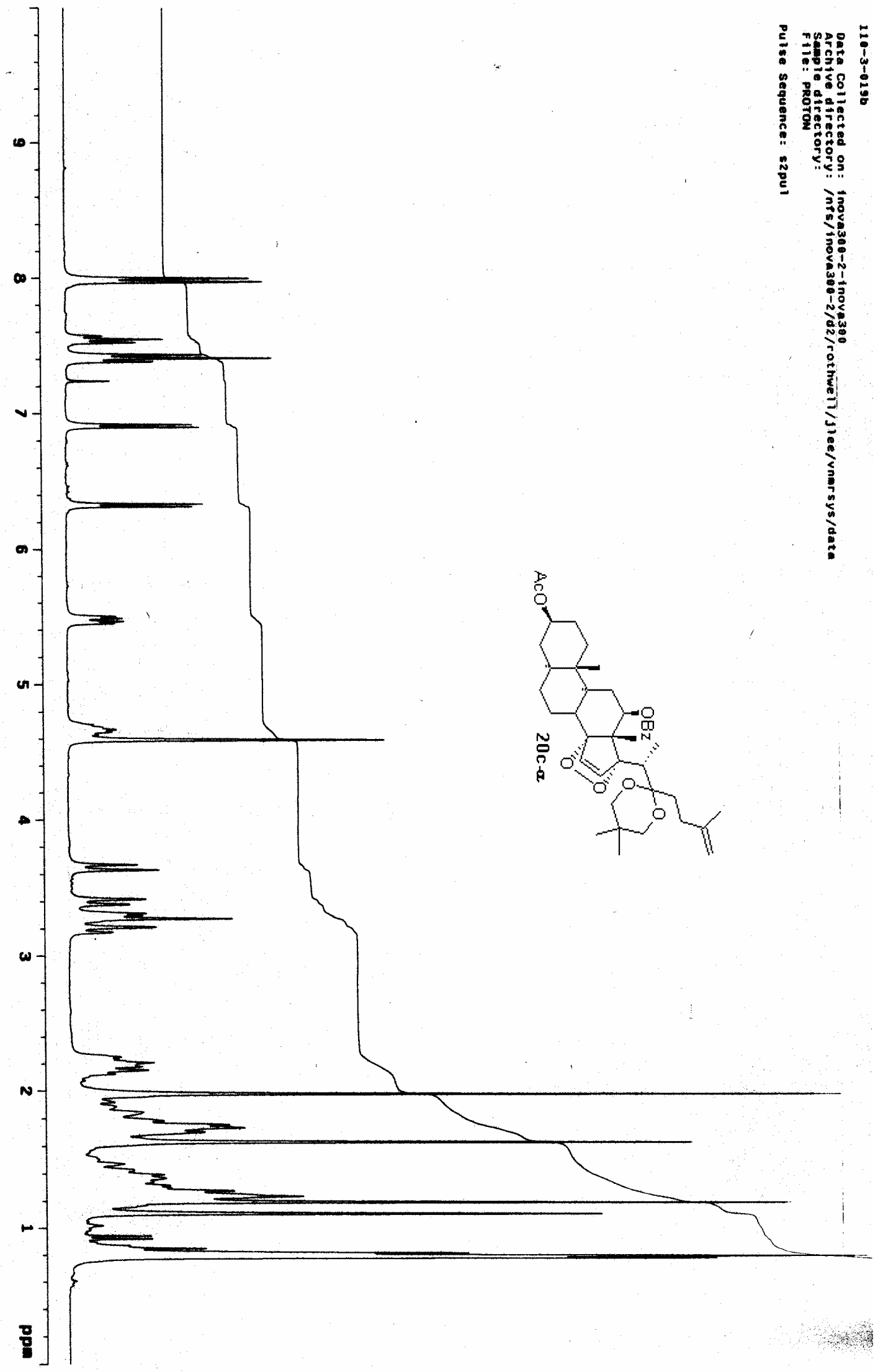



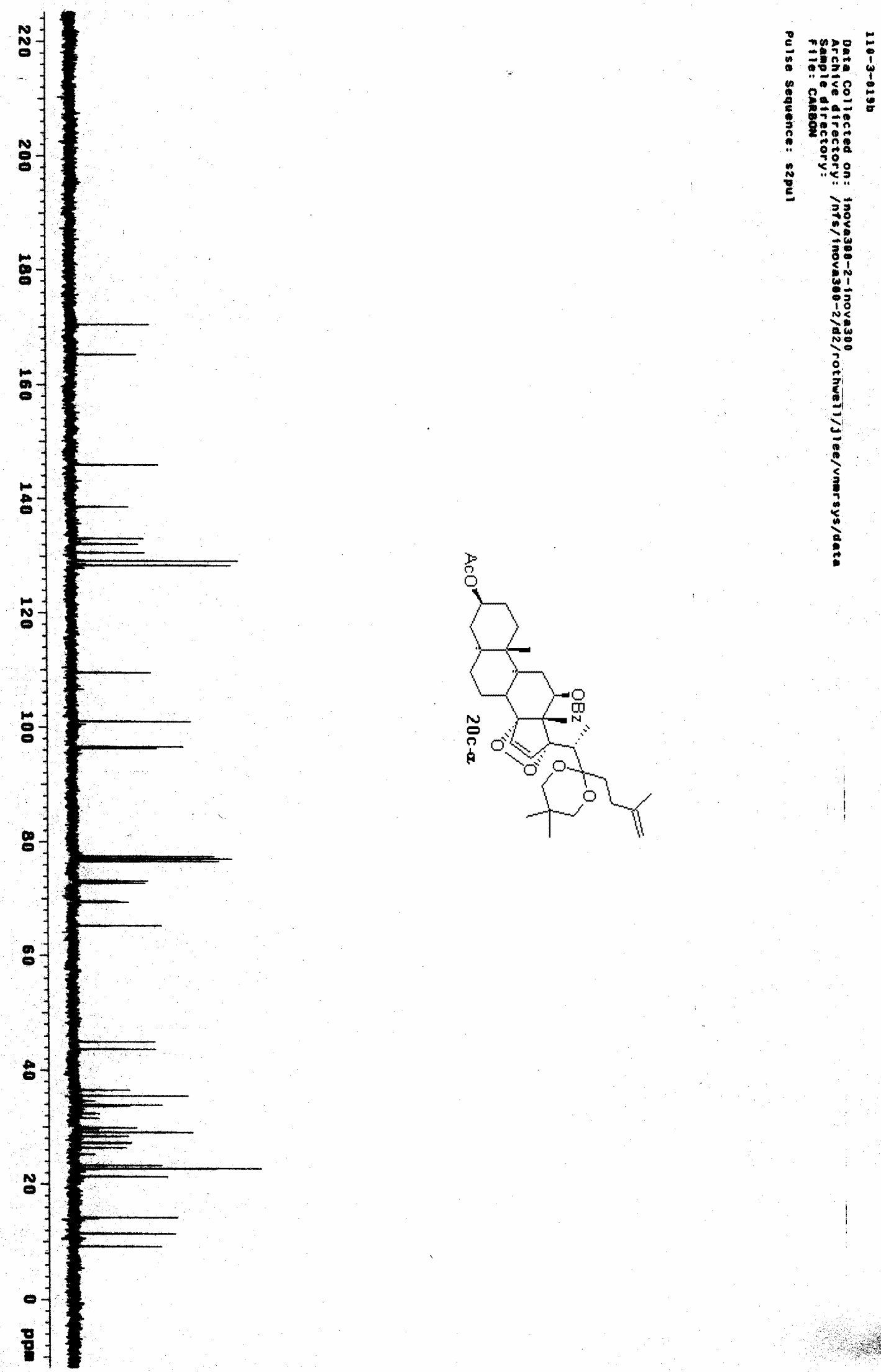


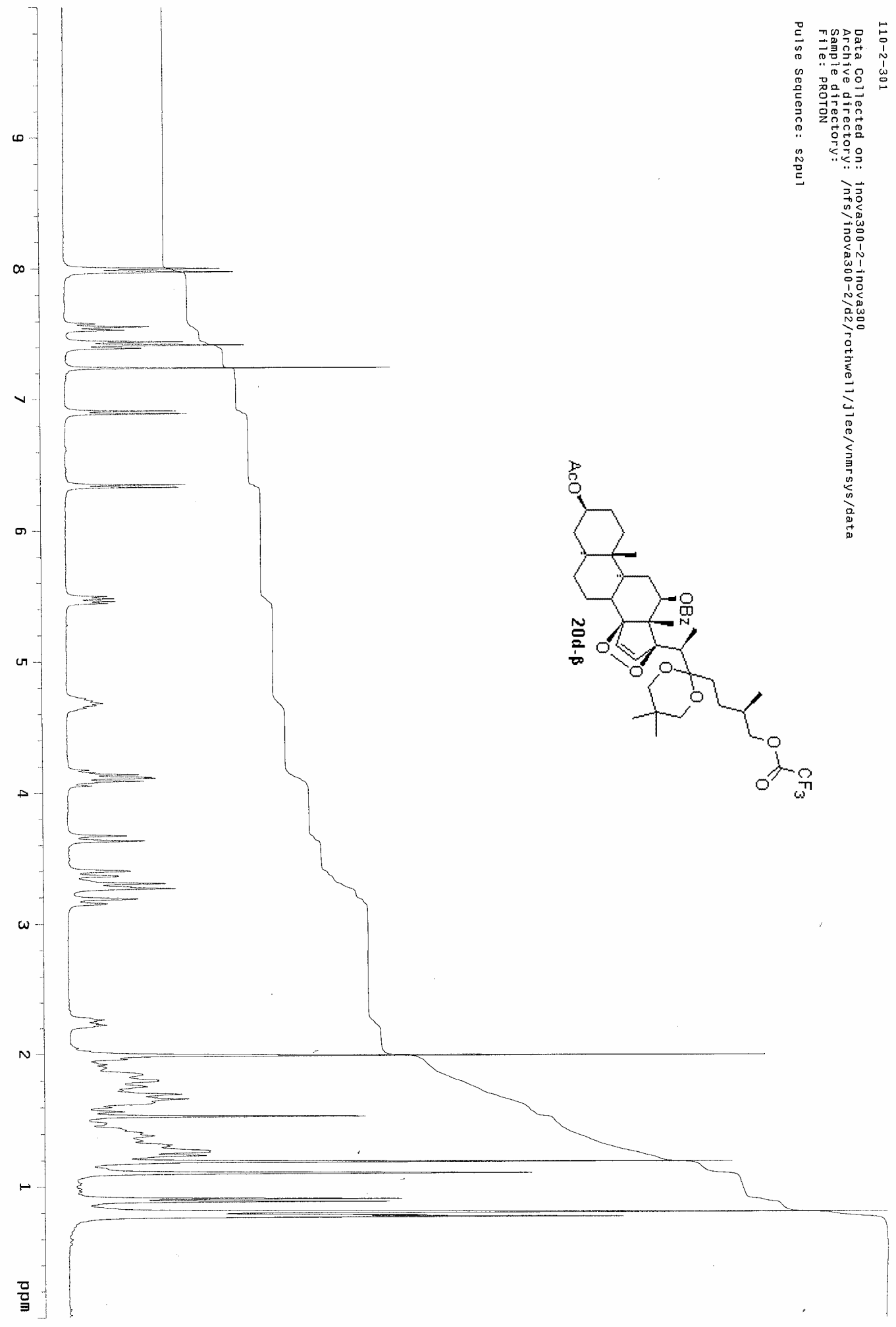

S- 50 

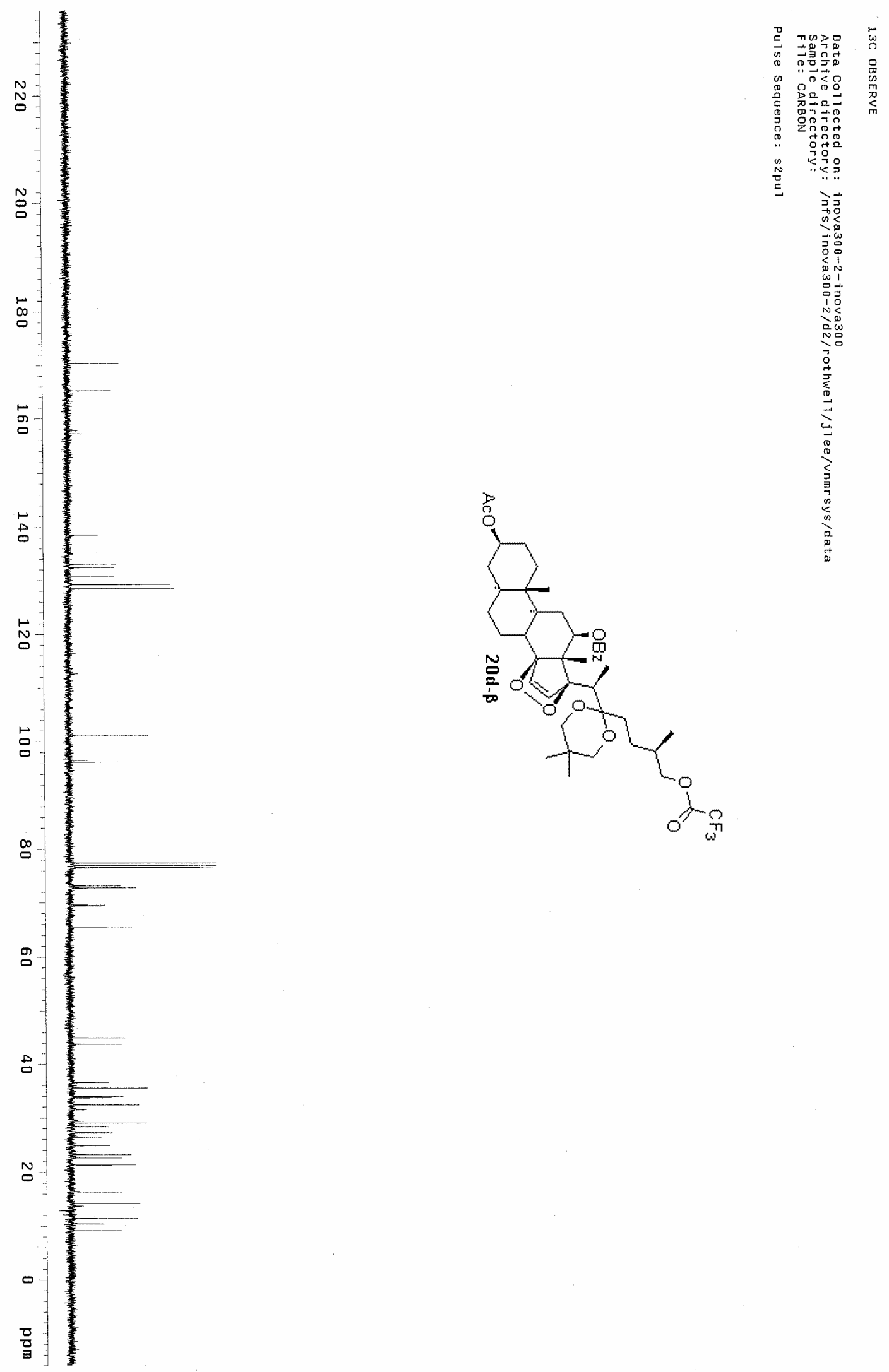


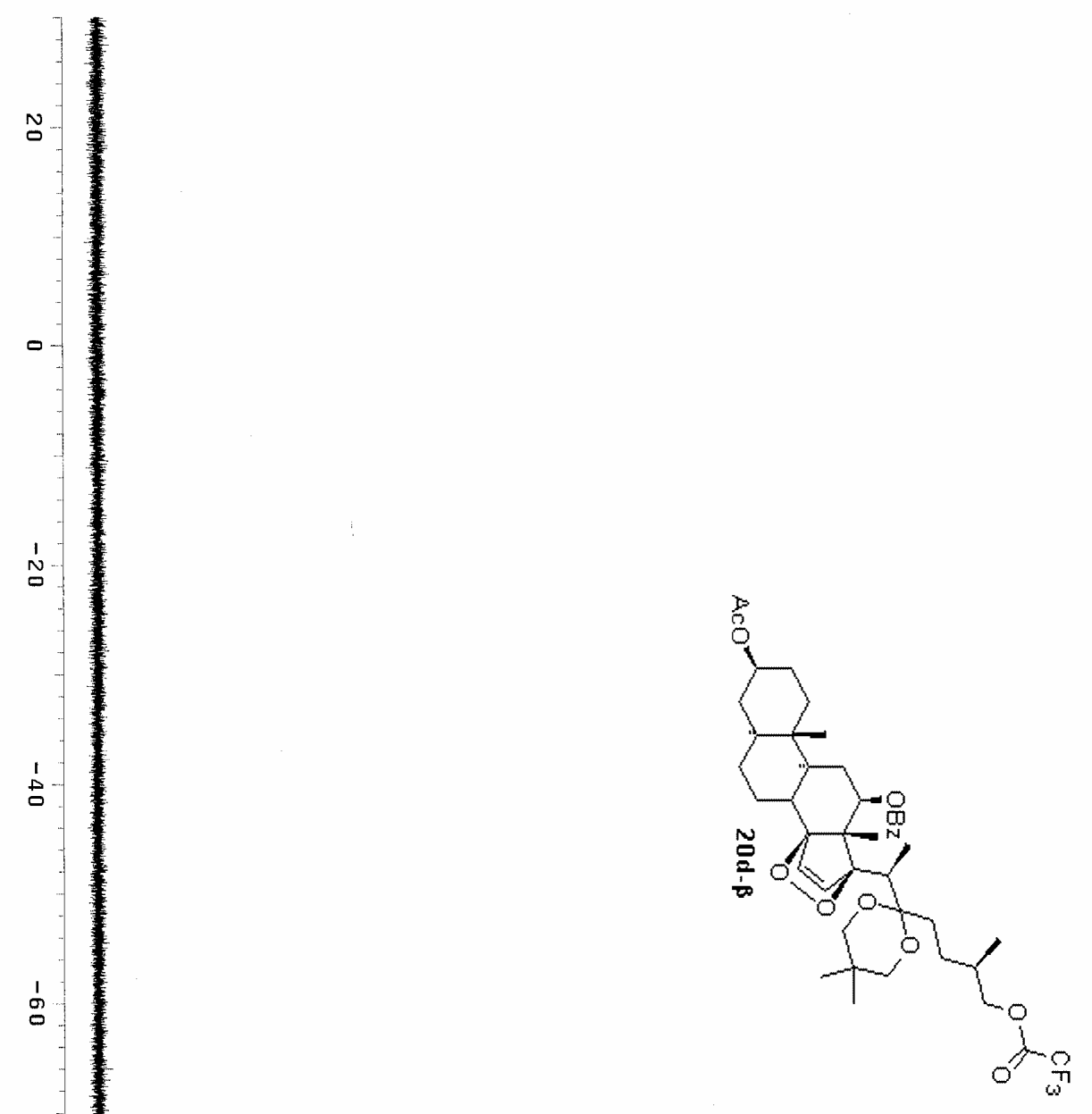

1
$\infty$
$\infty$

1.

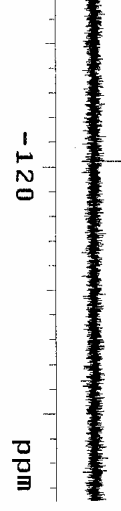

S- 52 


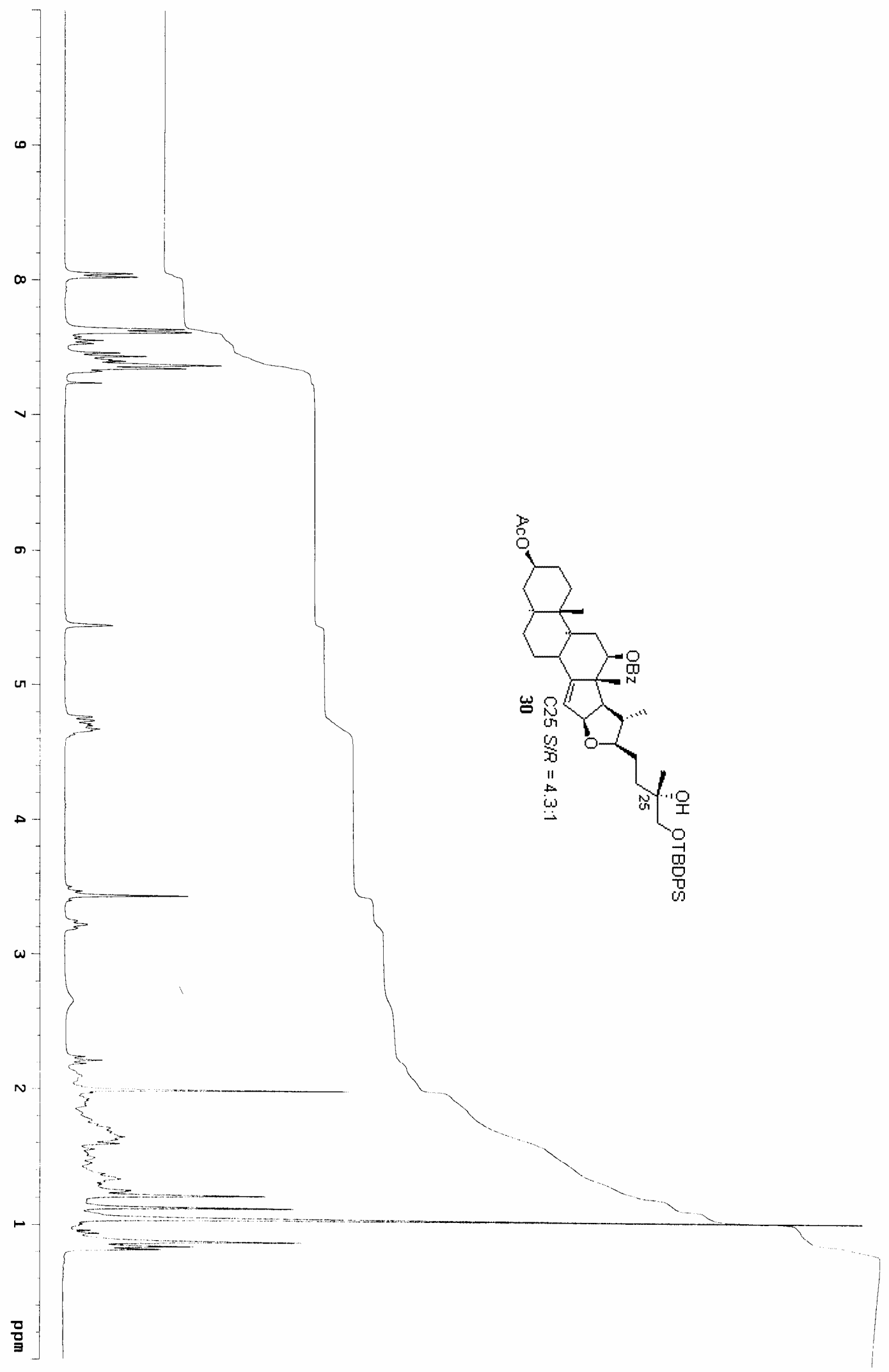

S- 53 

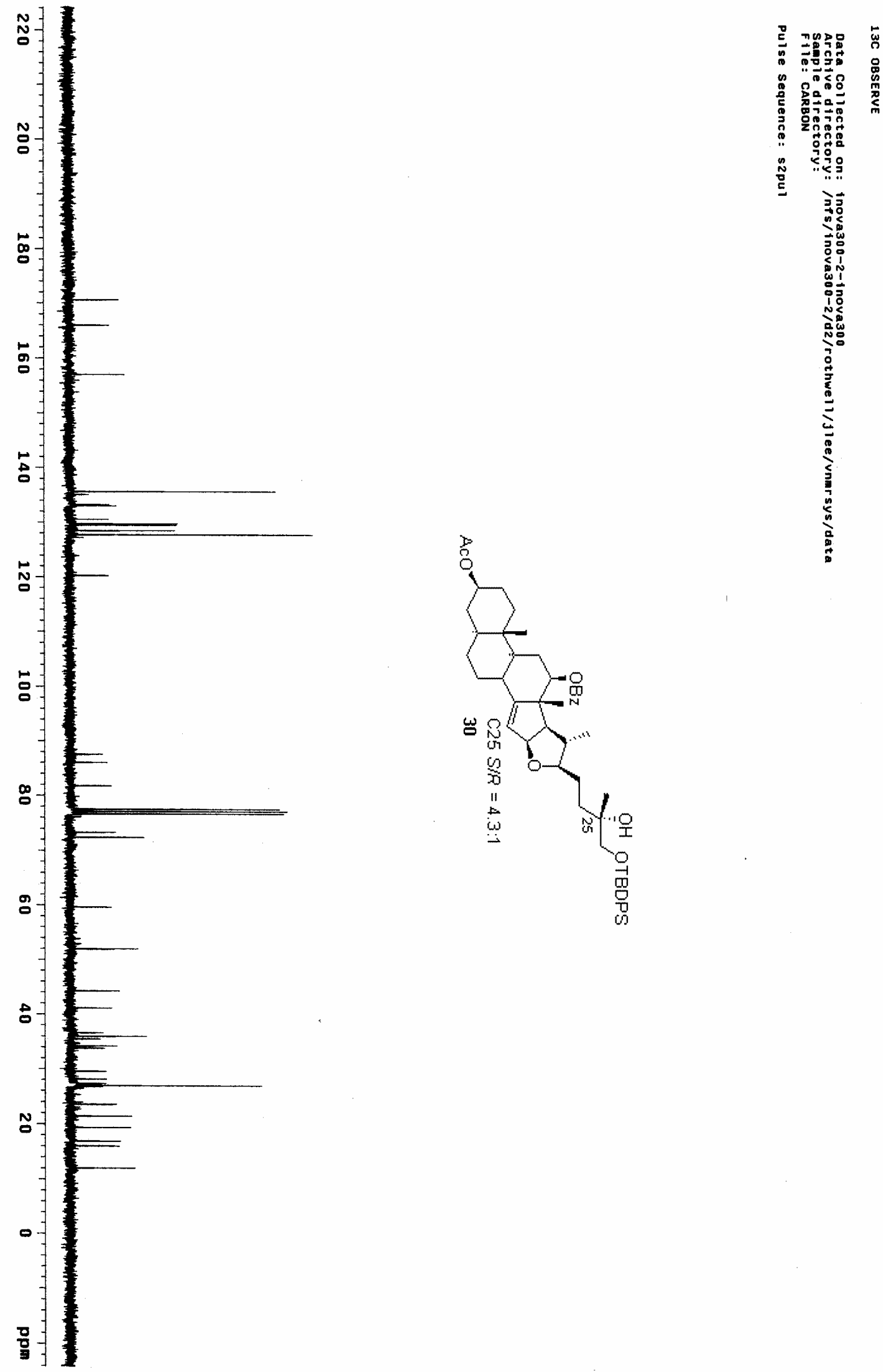


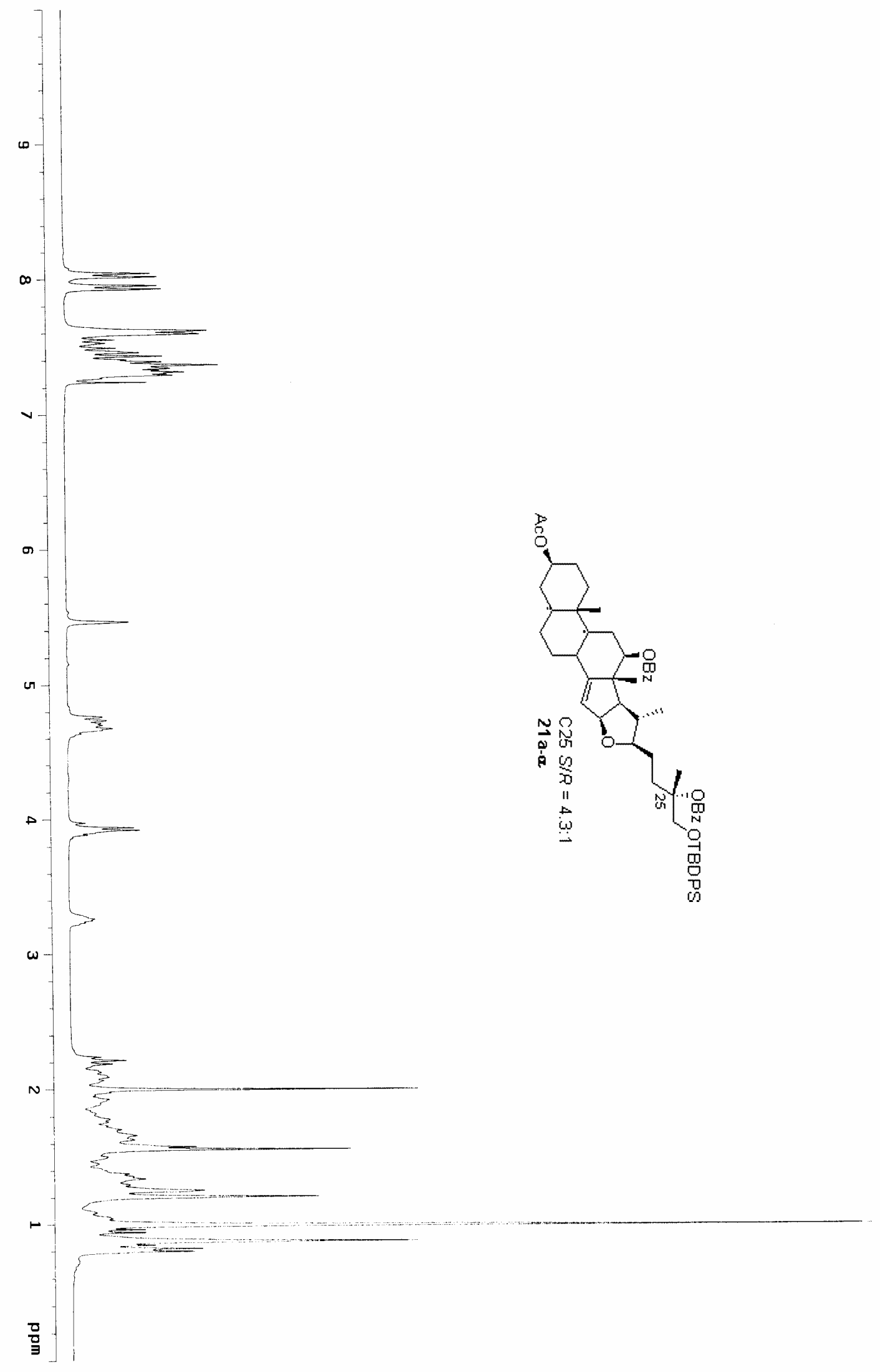

S- 55 

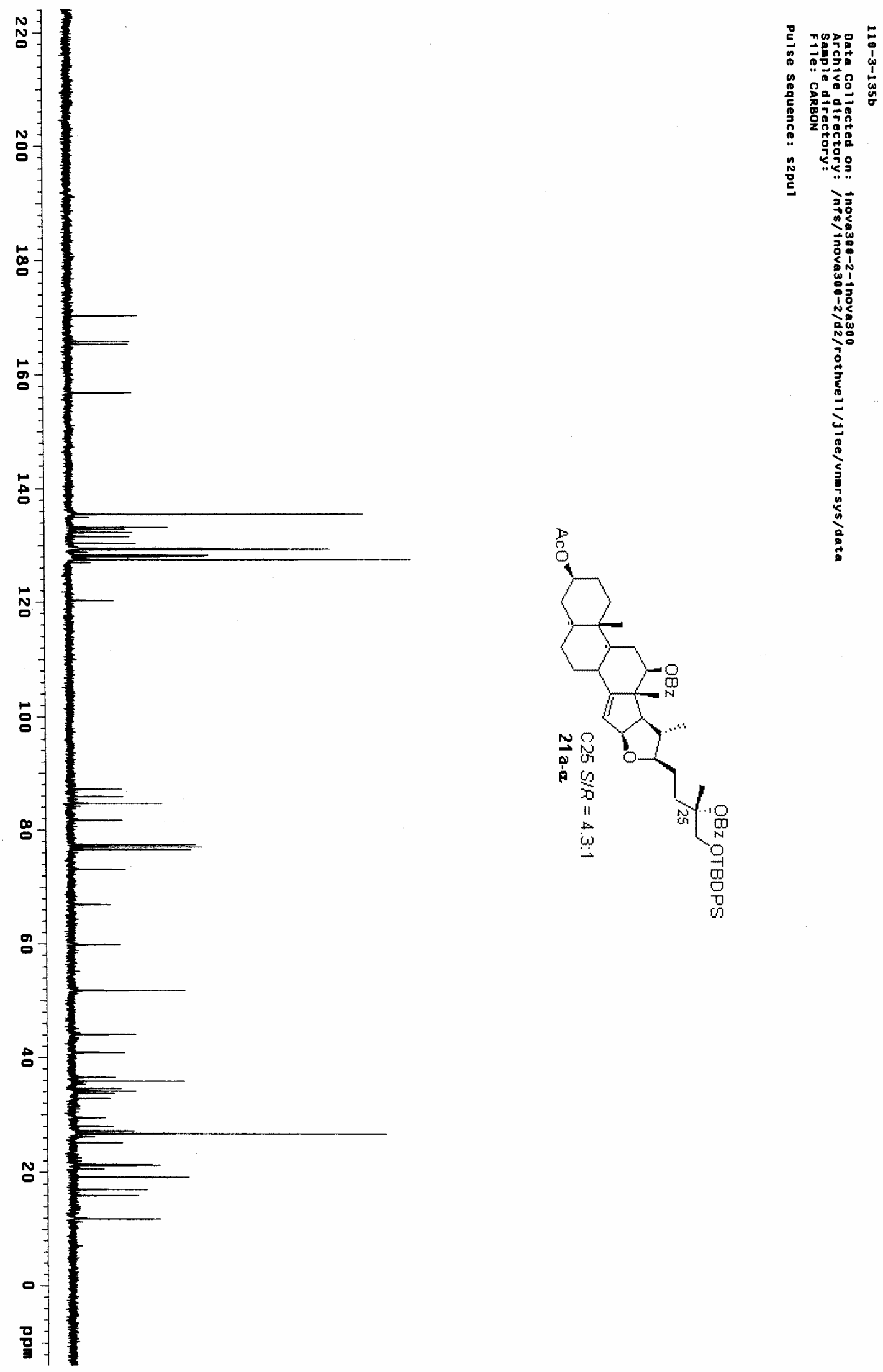

S- 56 


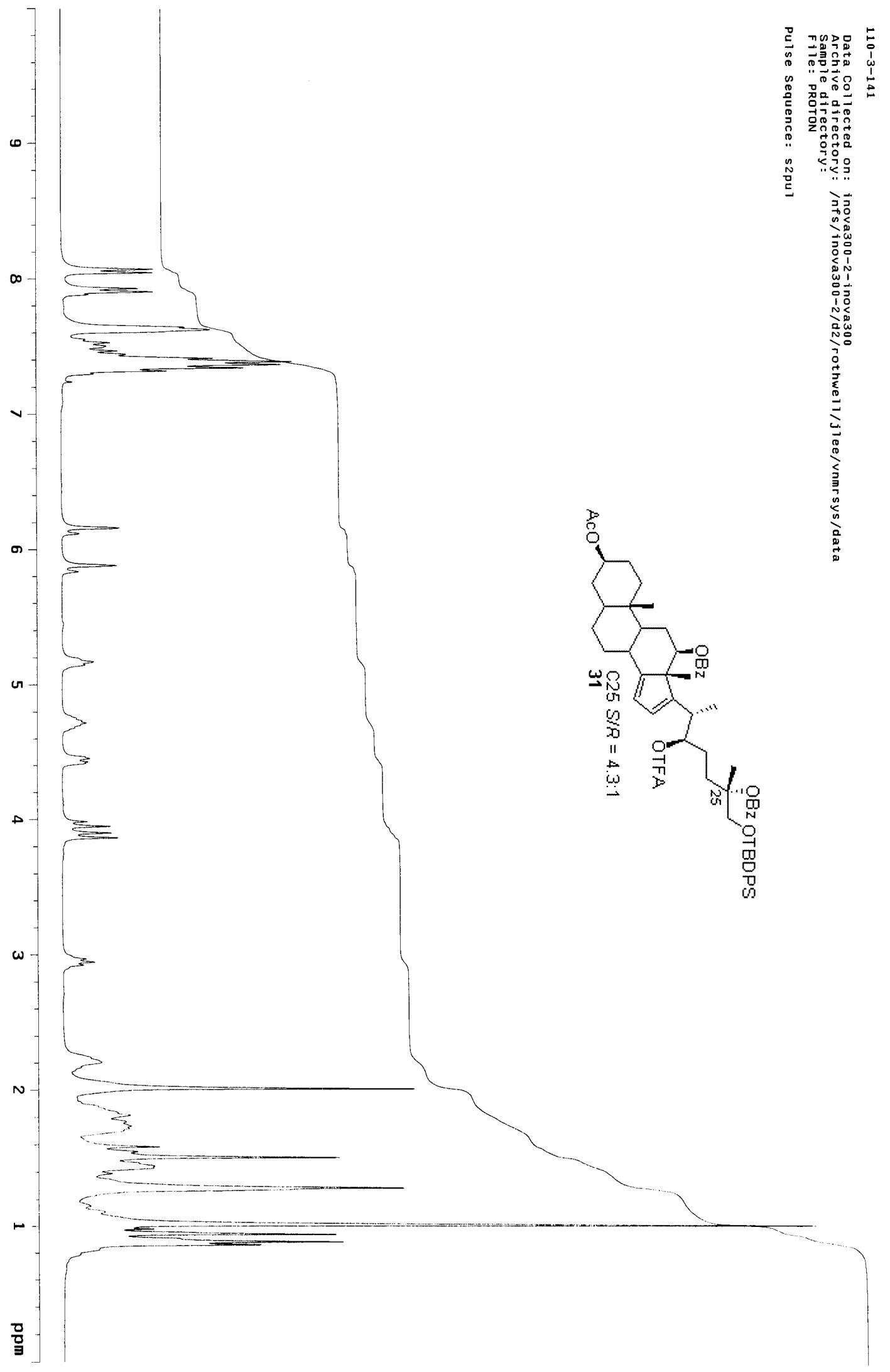



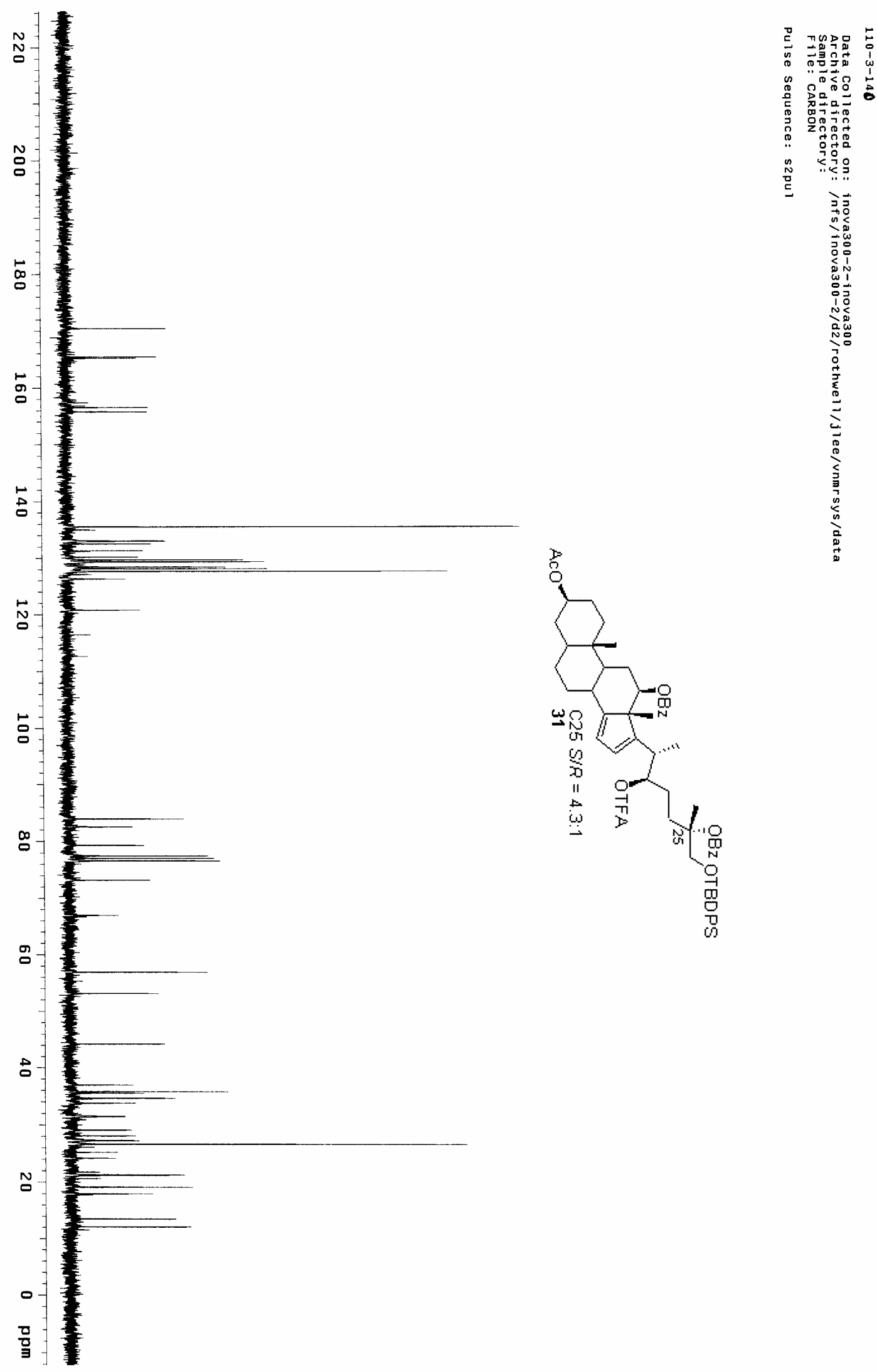

S- 58 


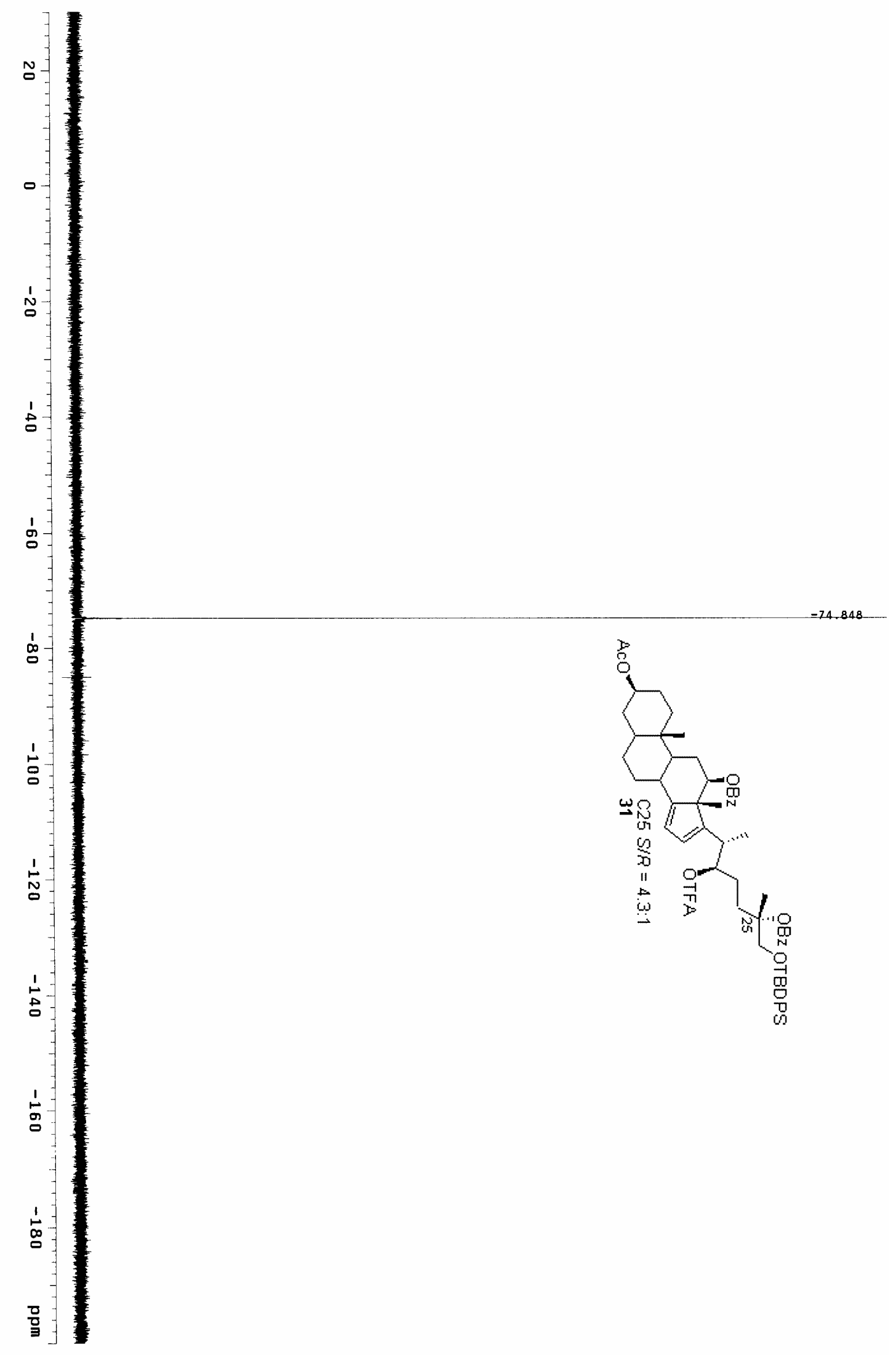




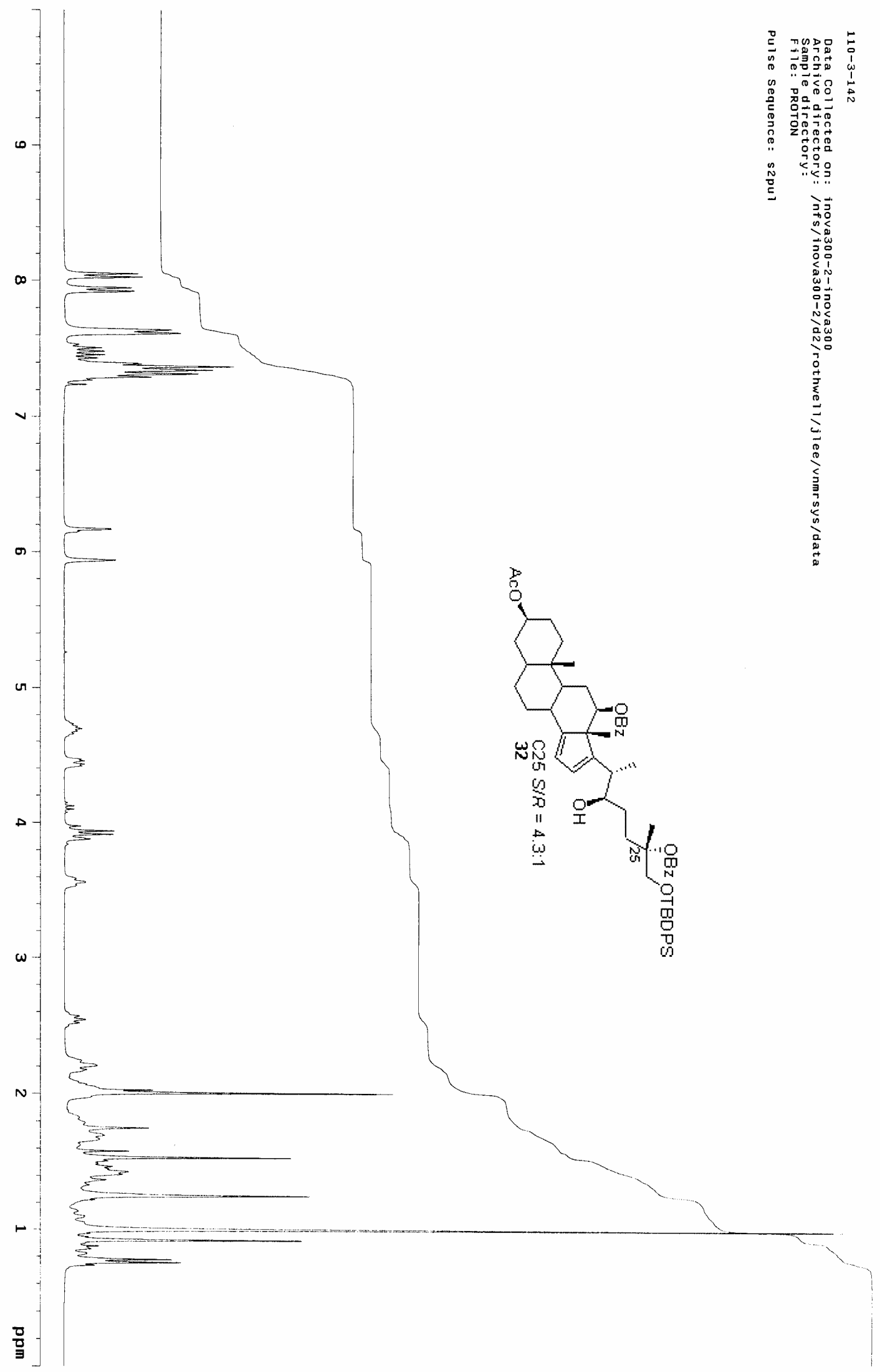

S- 60 

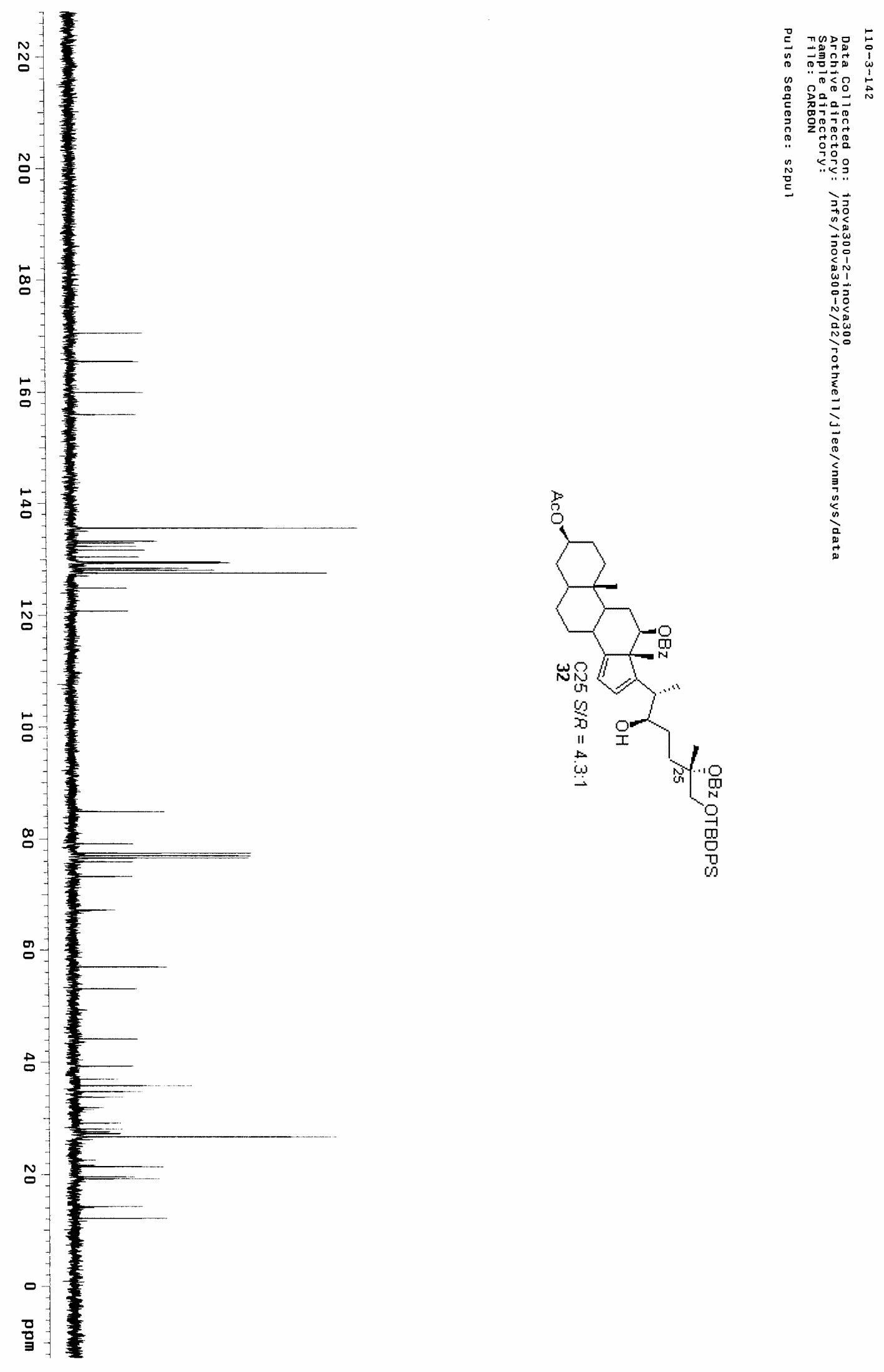

S- 61 


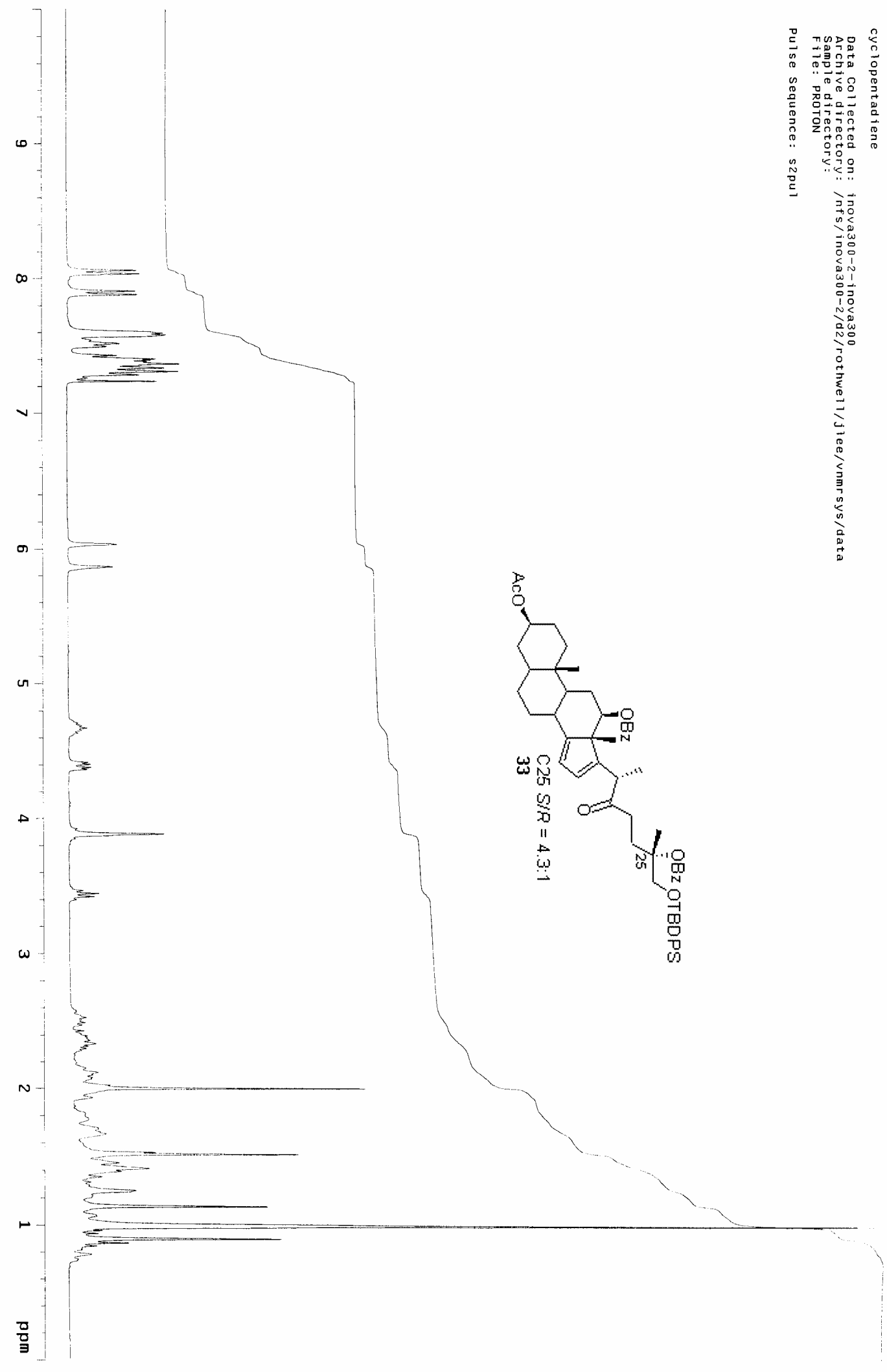

S- 62 


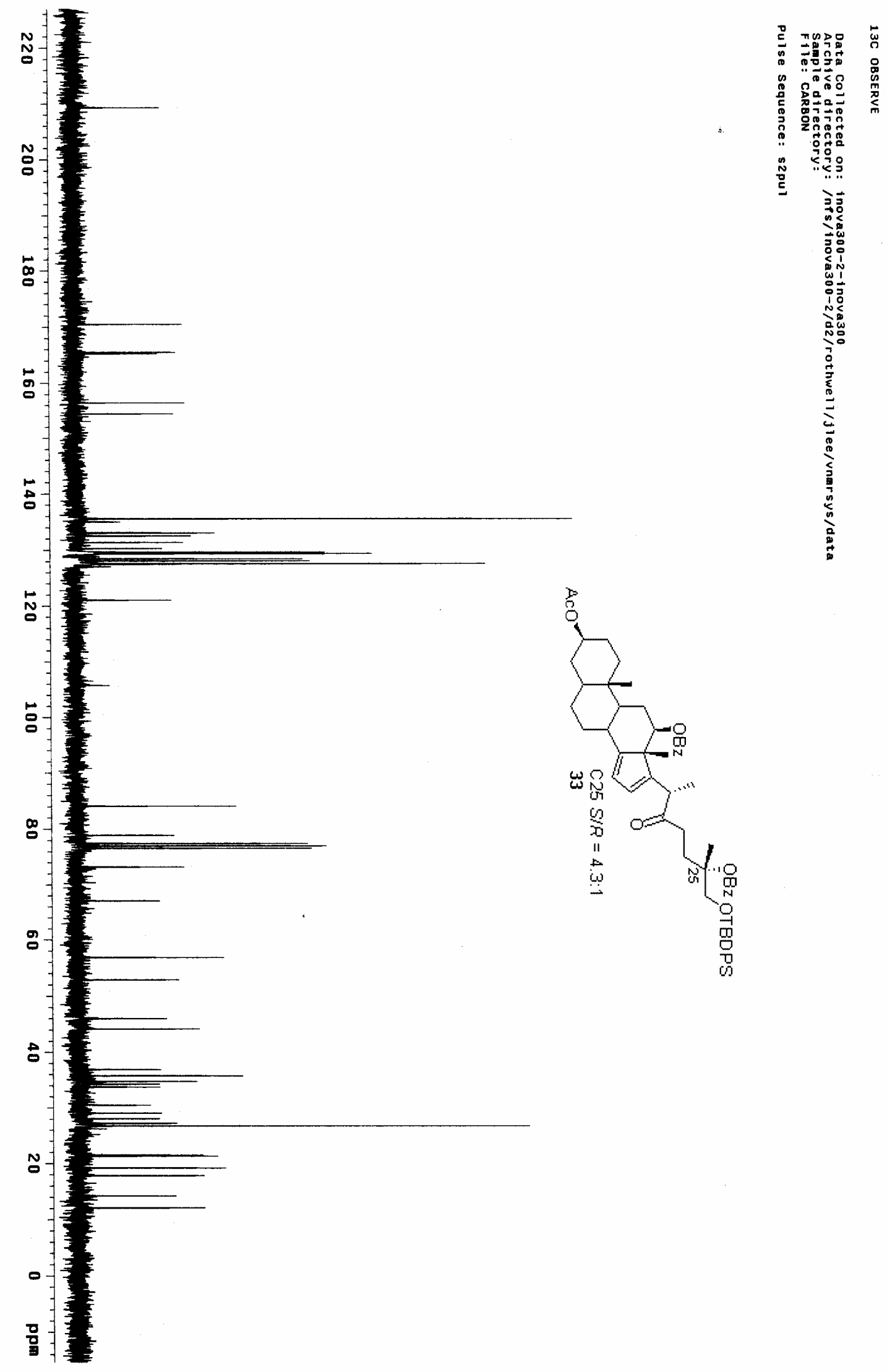

S- 63 


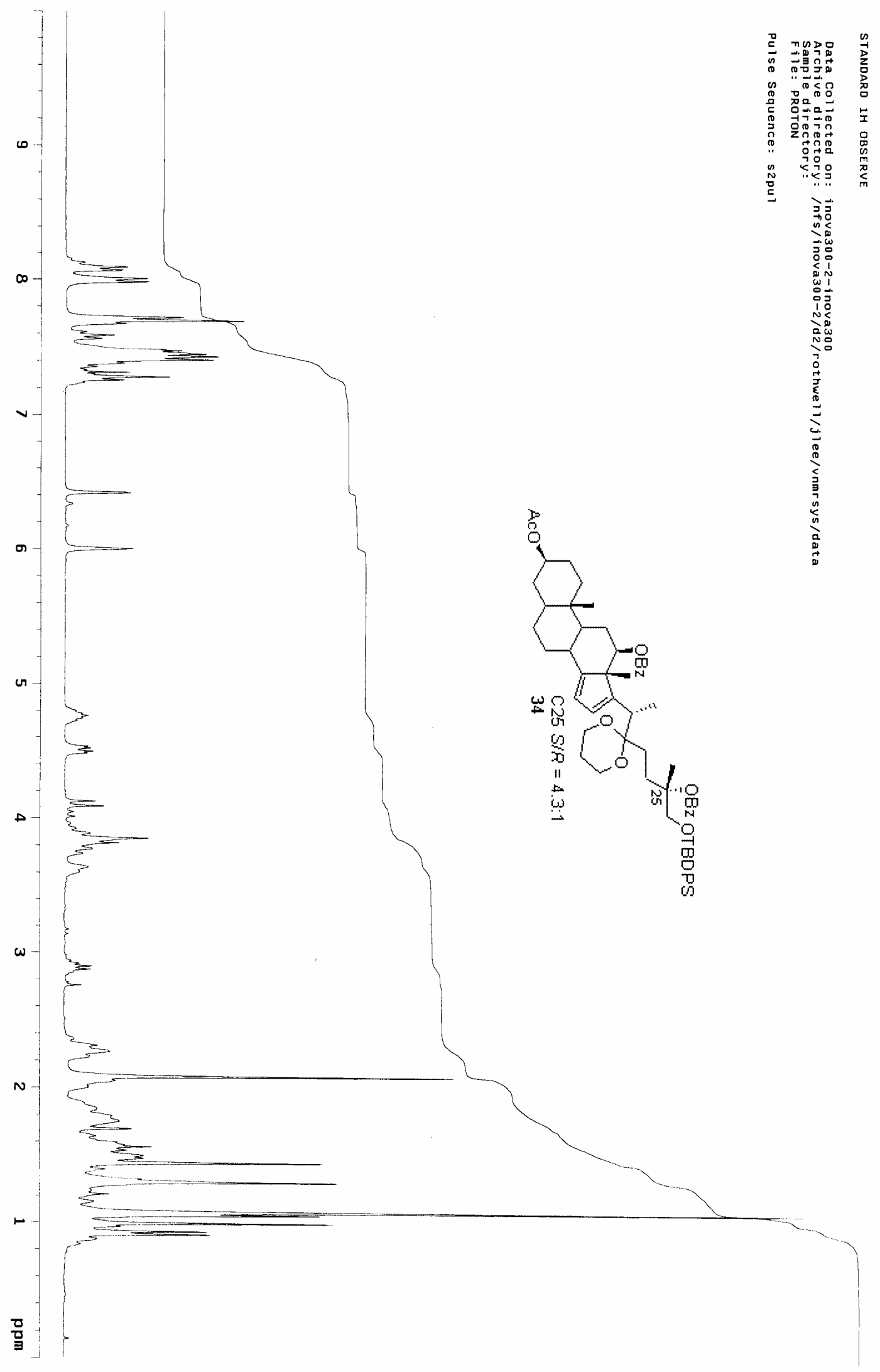

S- 64 


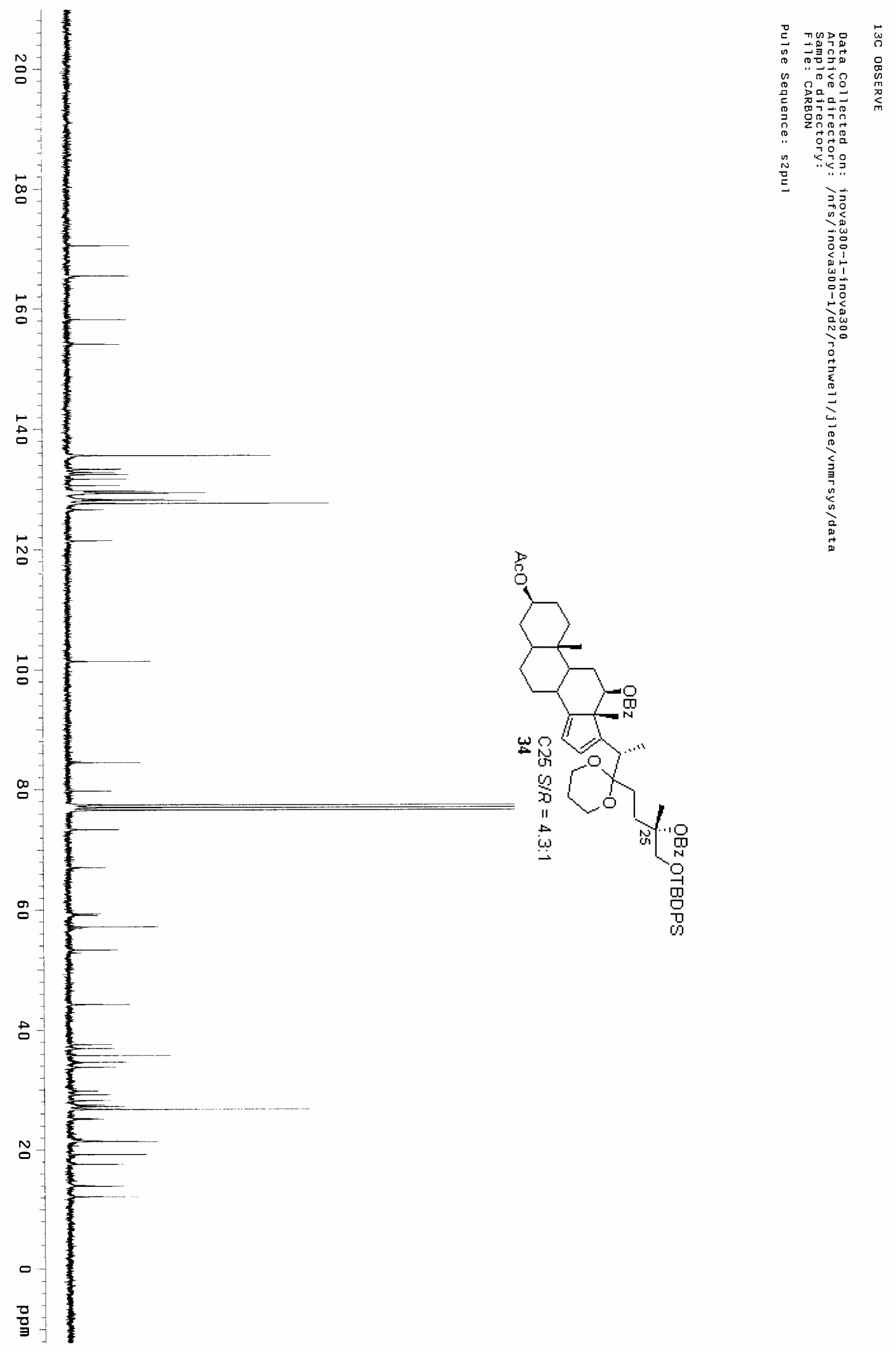




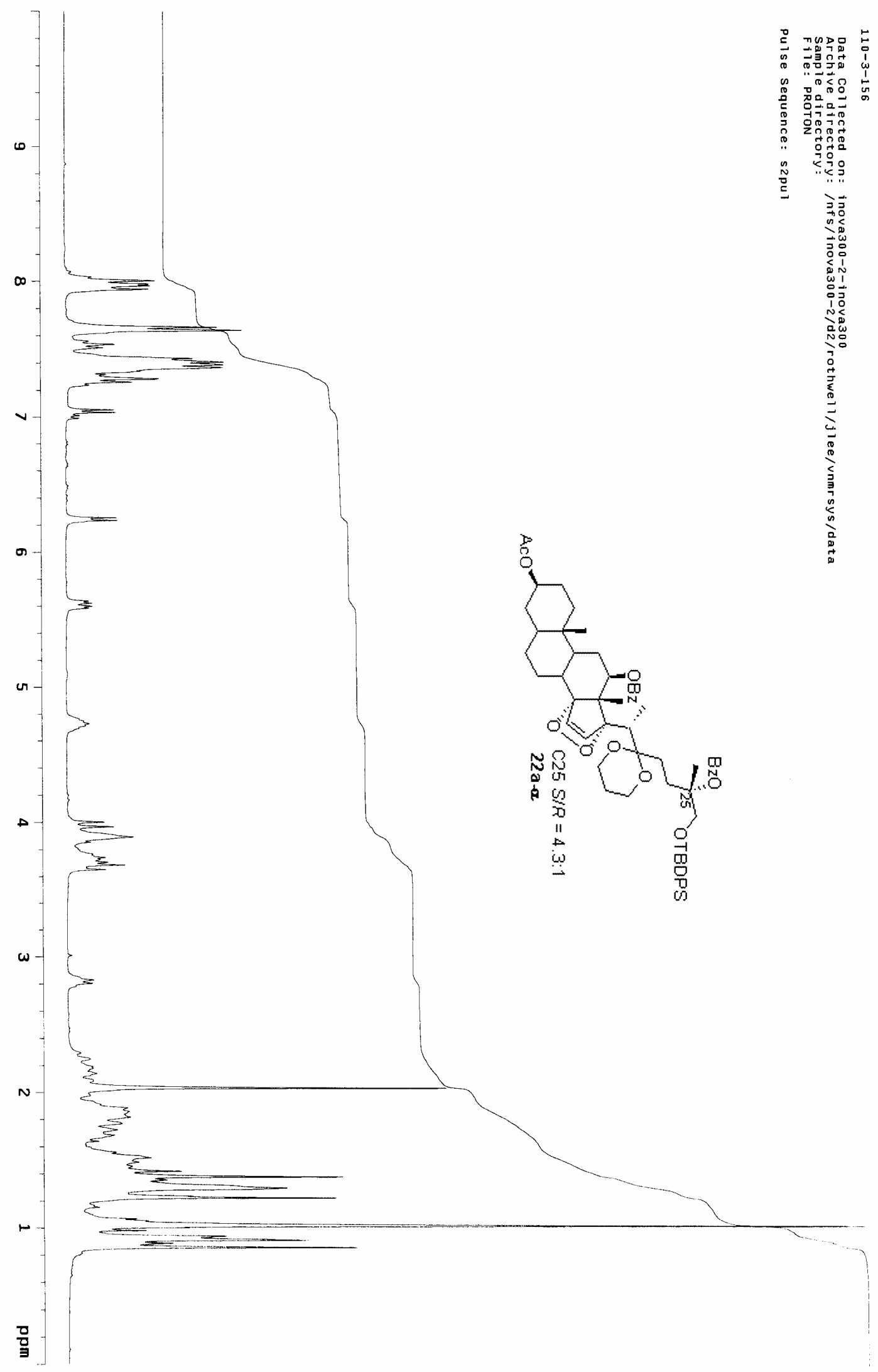

S- 66 

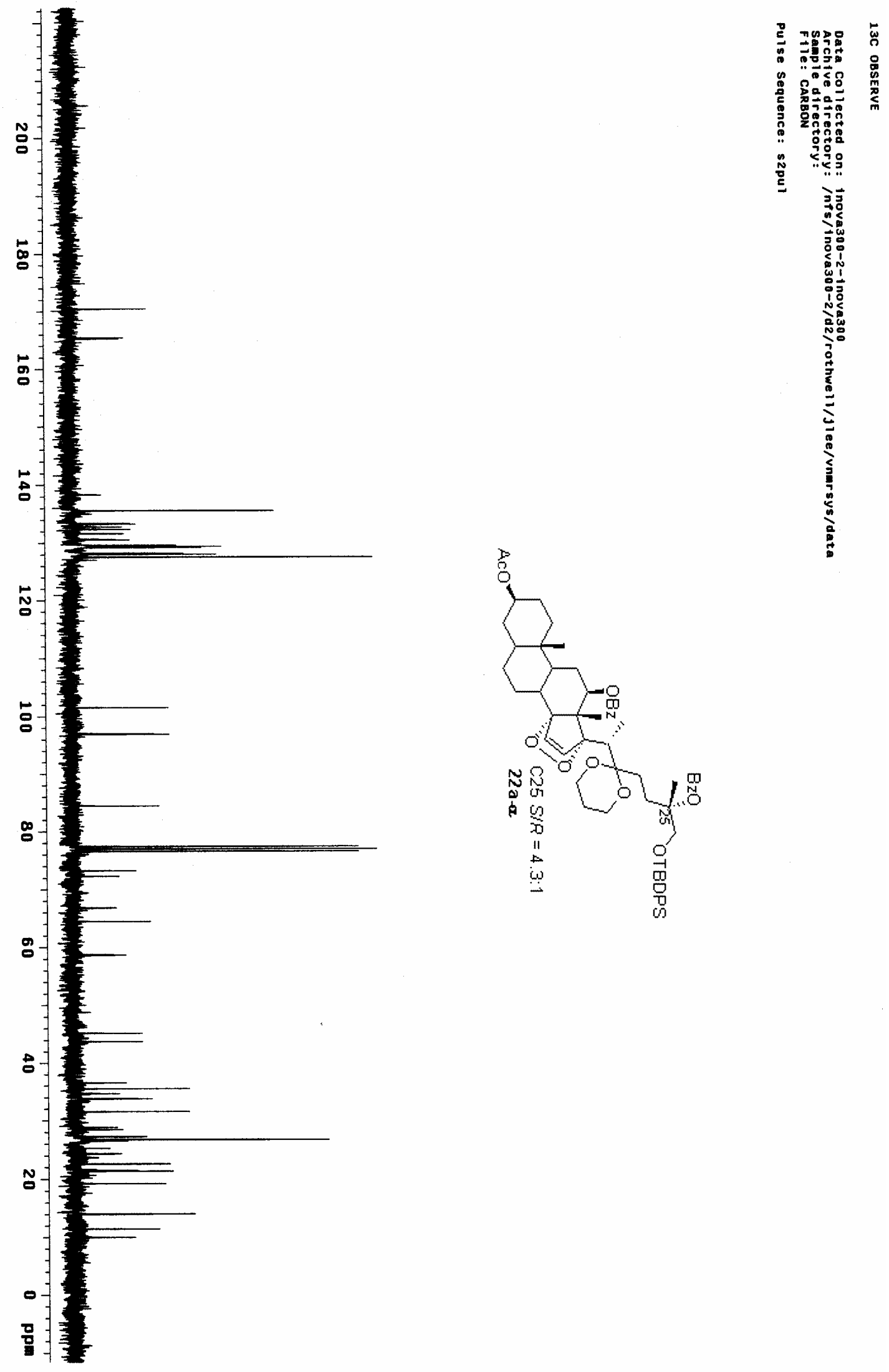

S- 67 


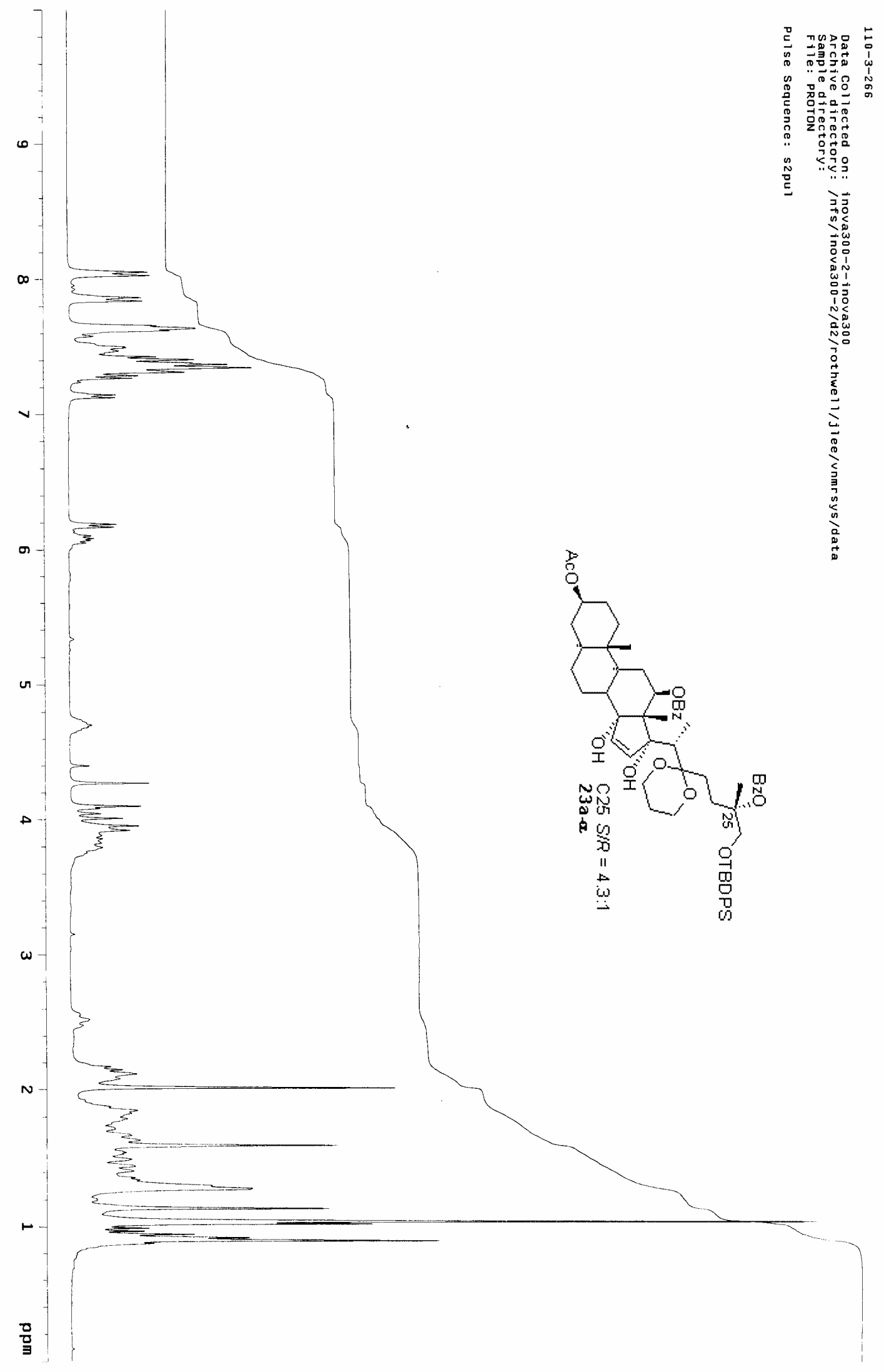

S- 68 

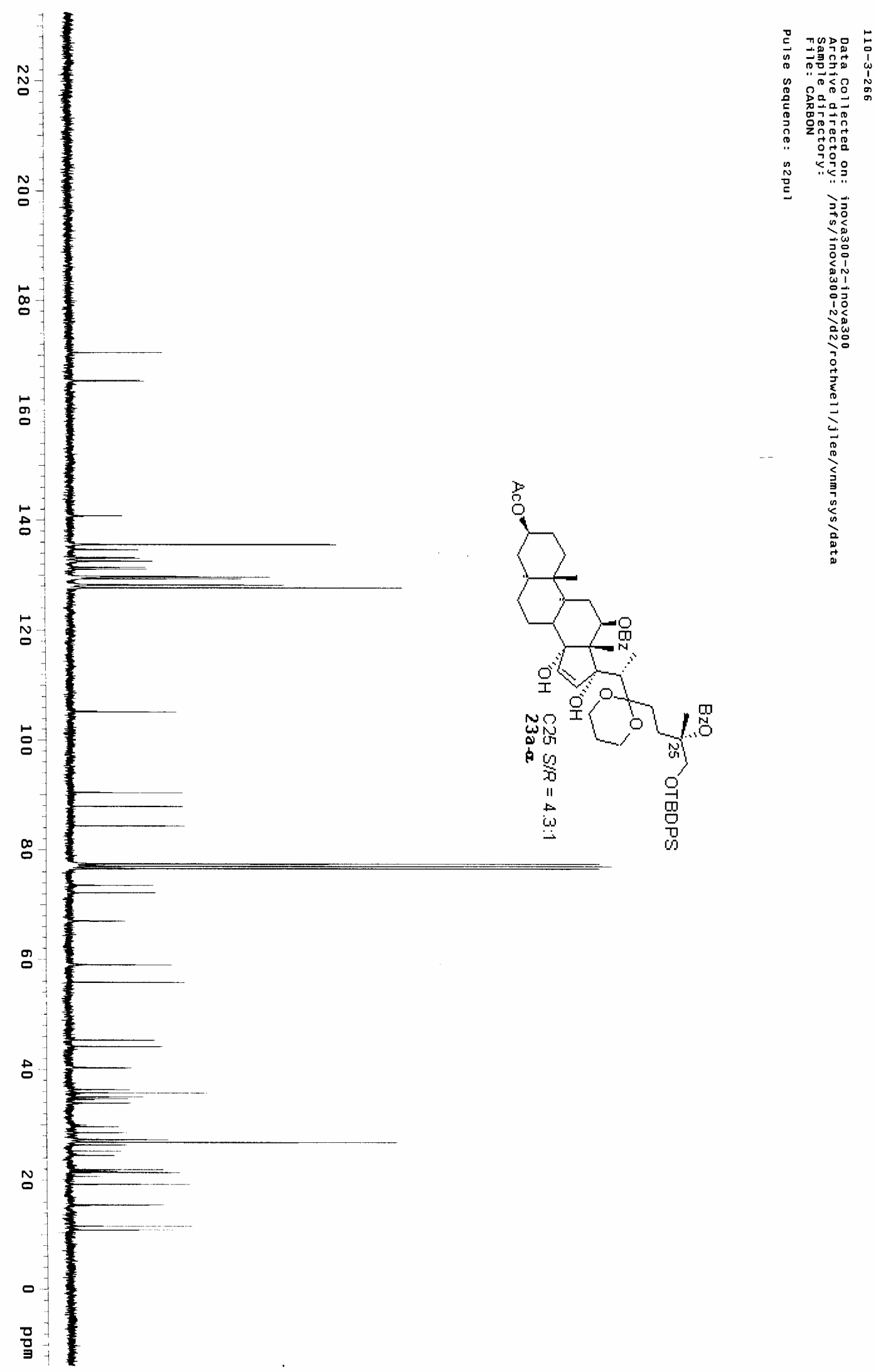


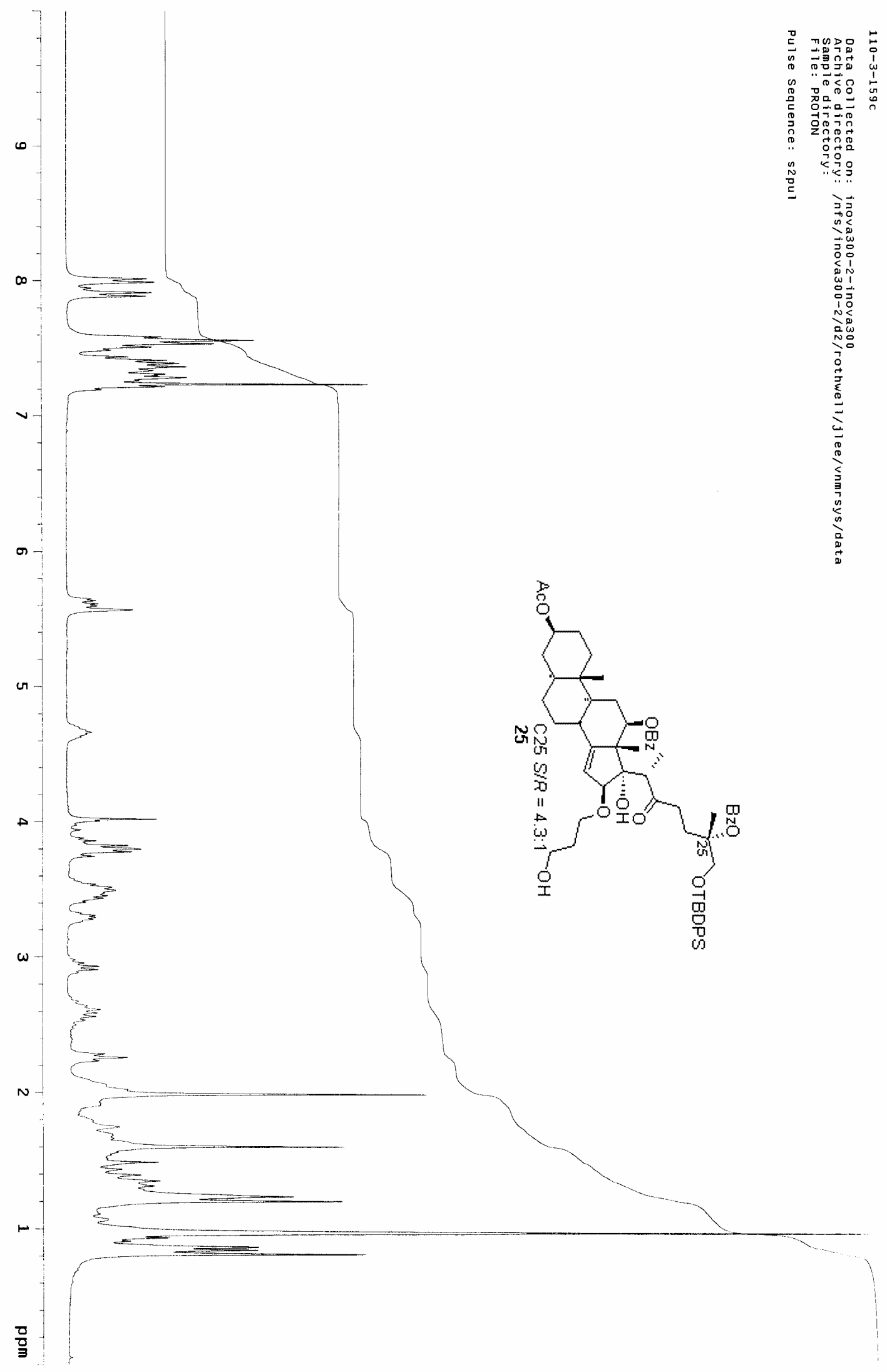

S- 70 

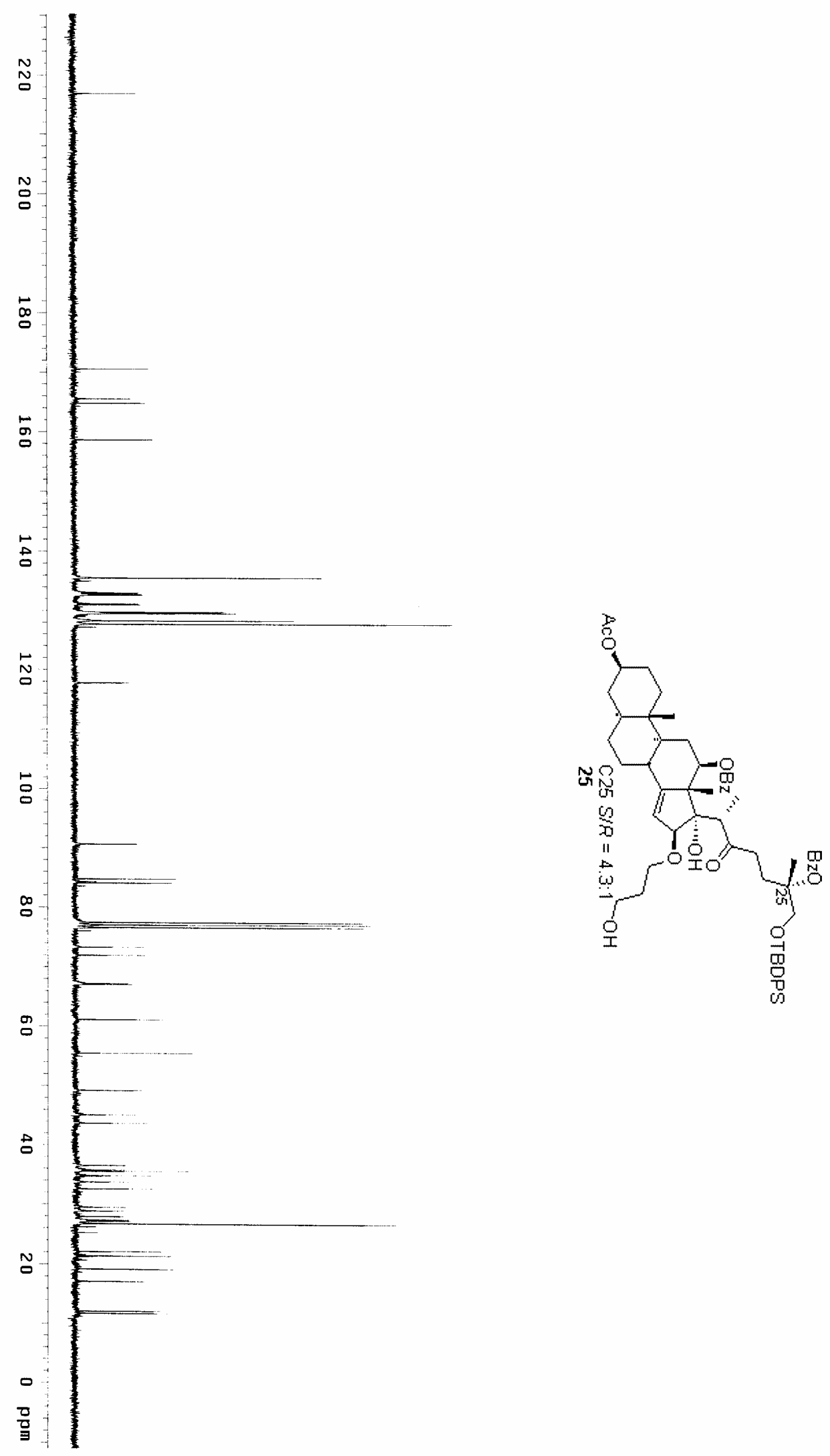


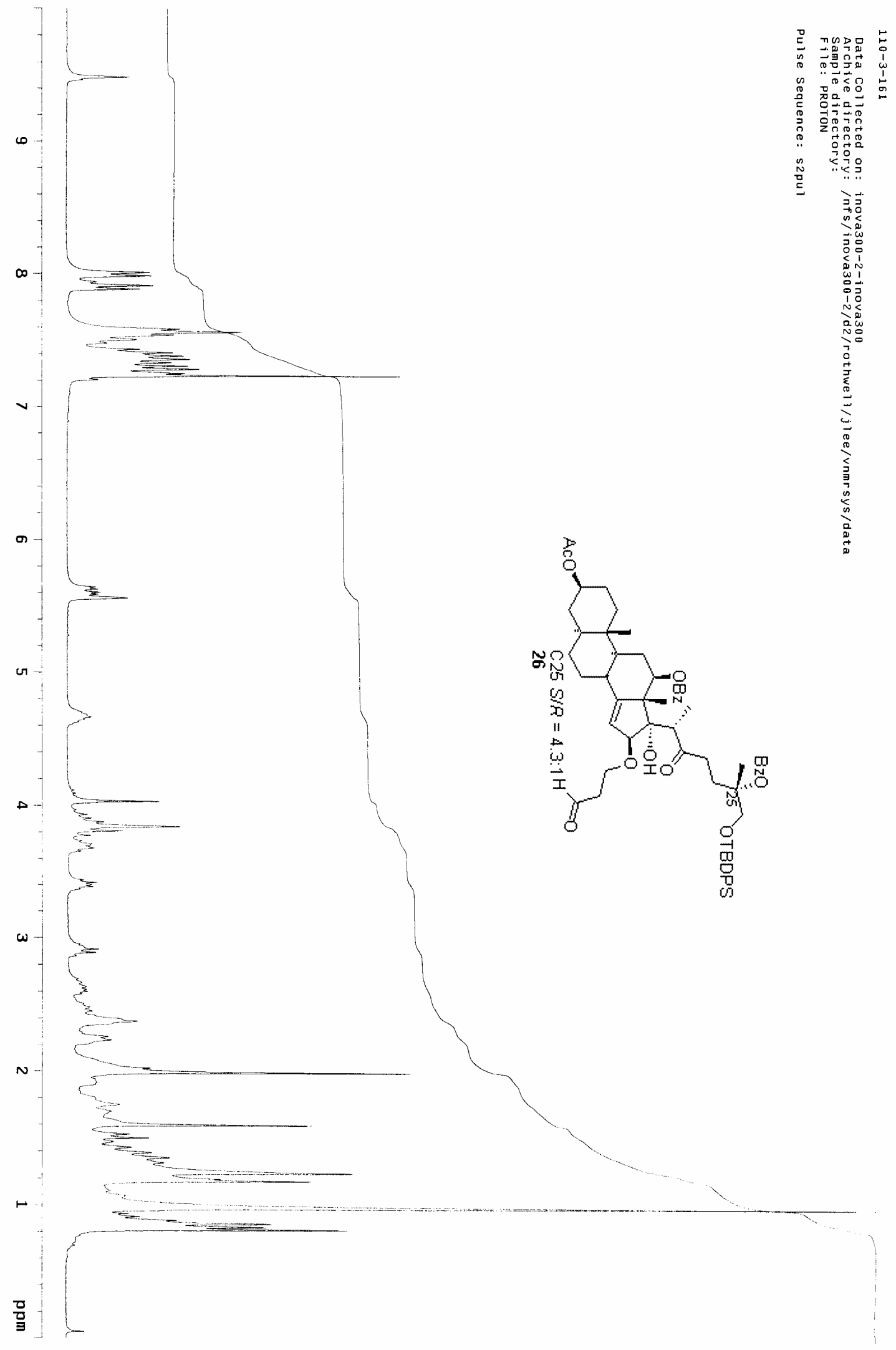

S- 72 

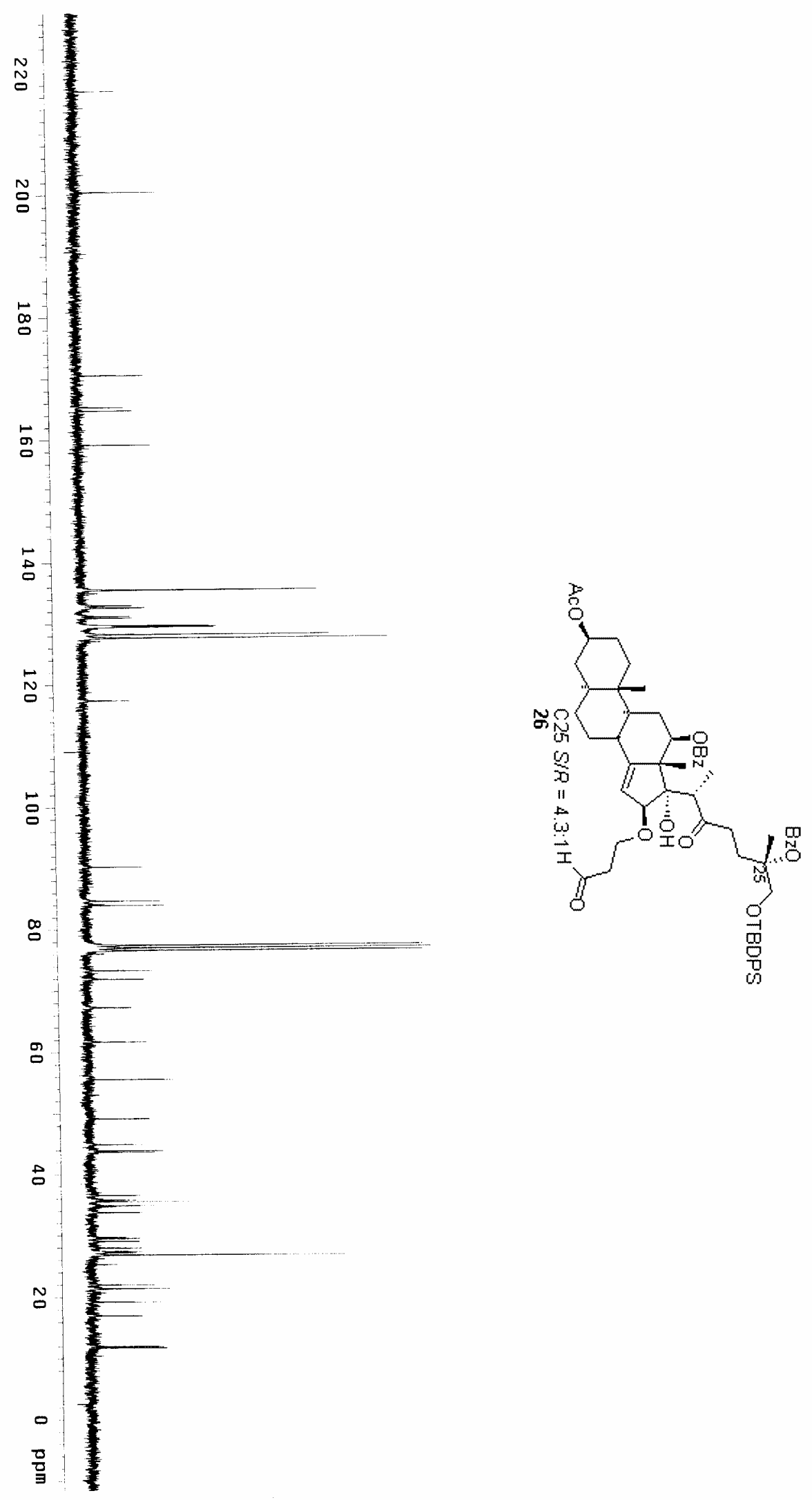

S- 73 


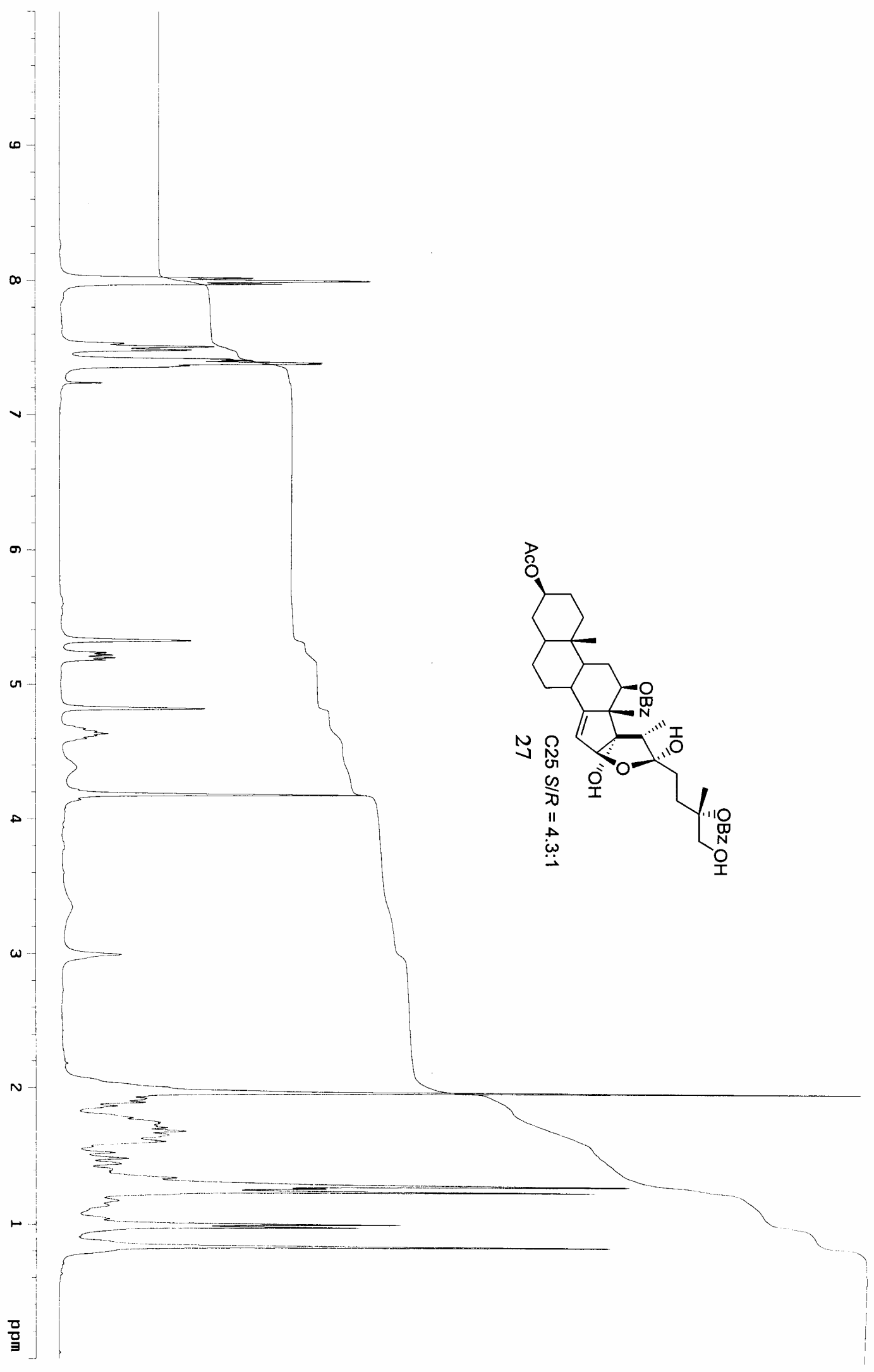

S- 74 

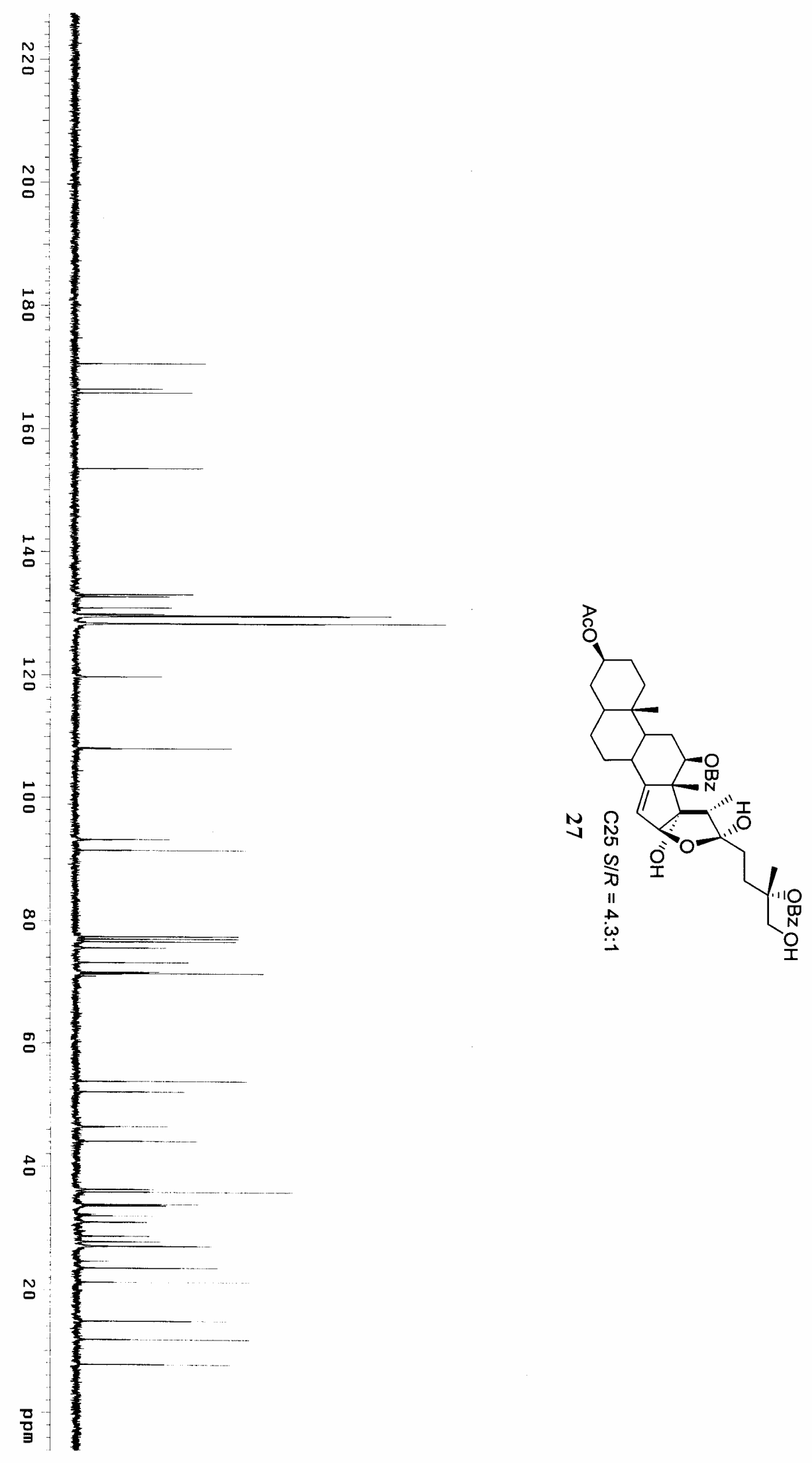


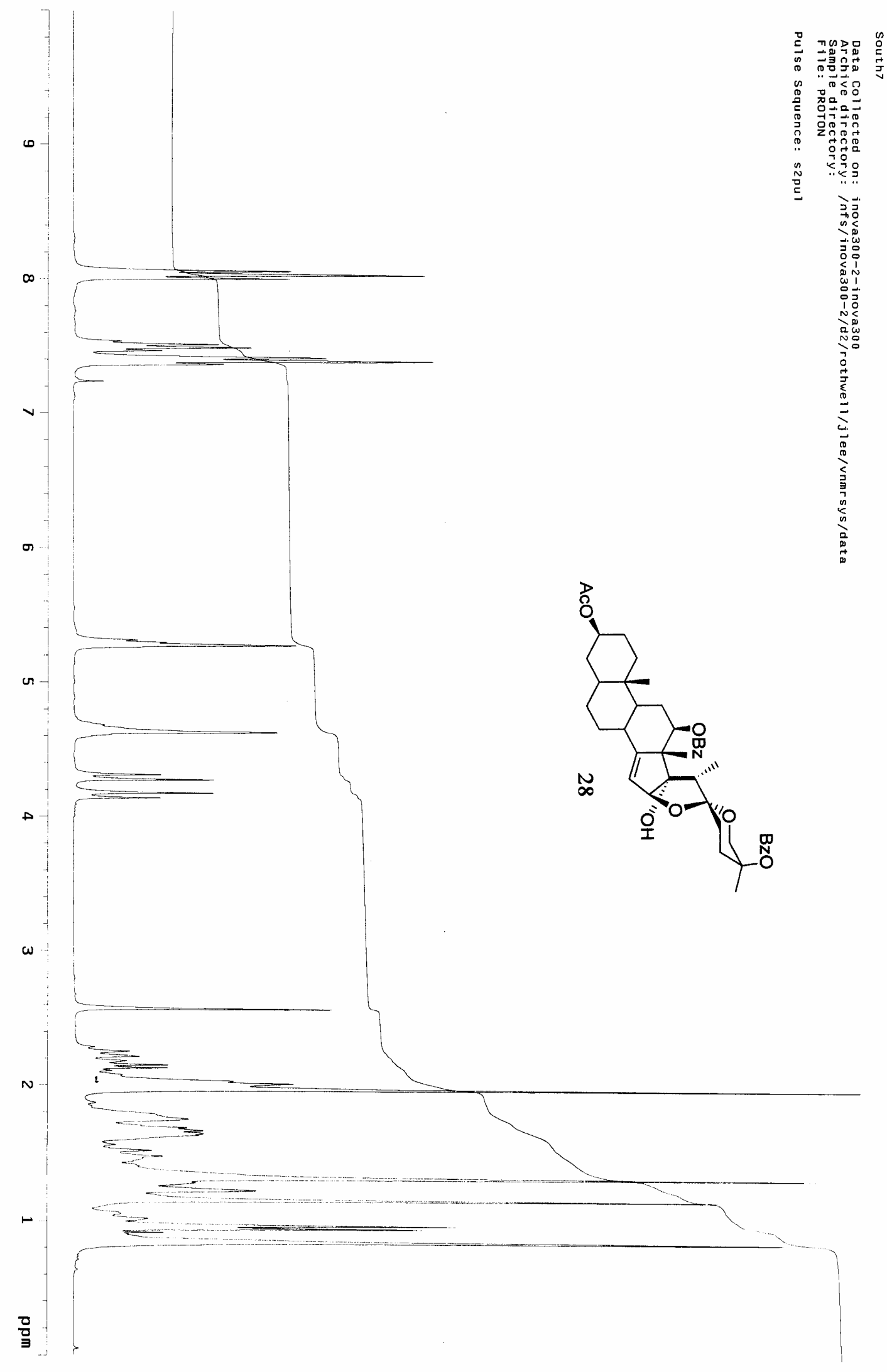

S- 76 


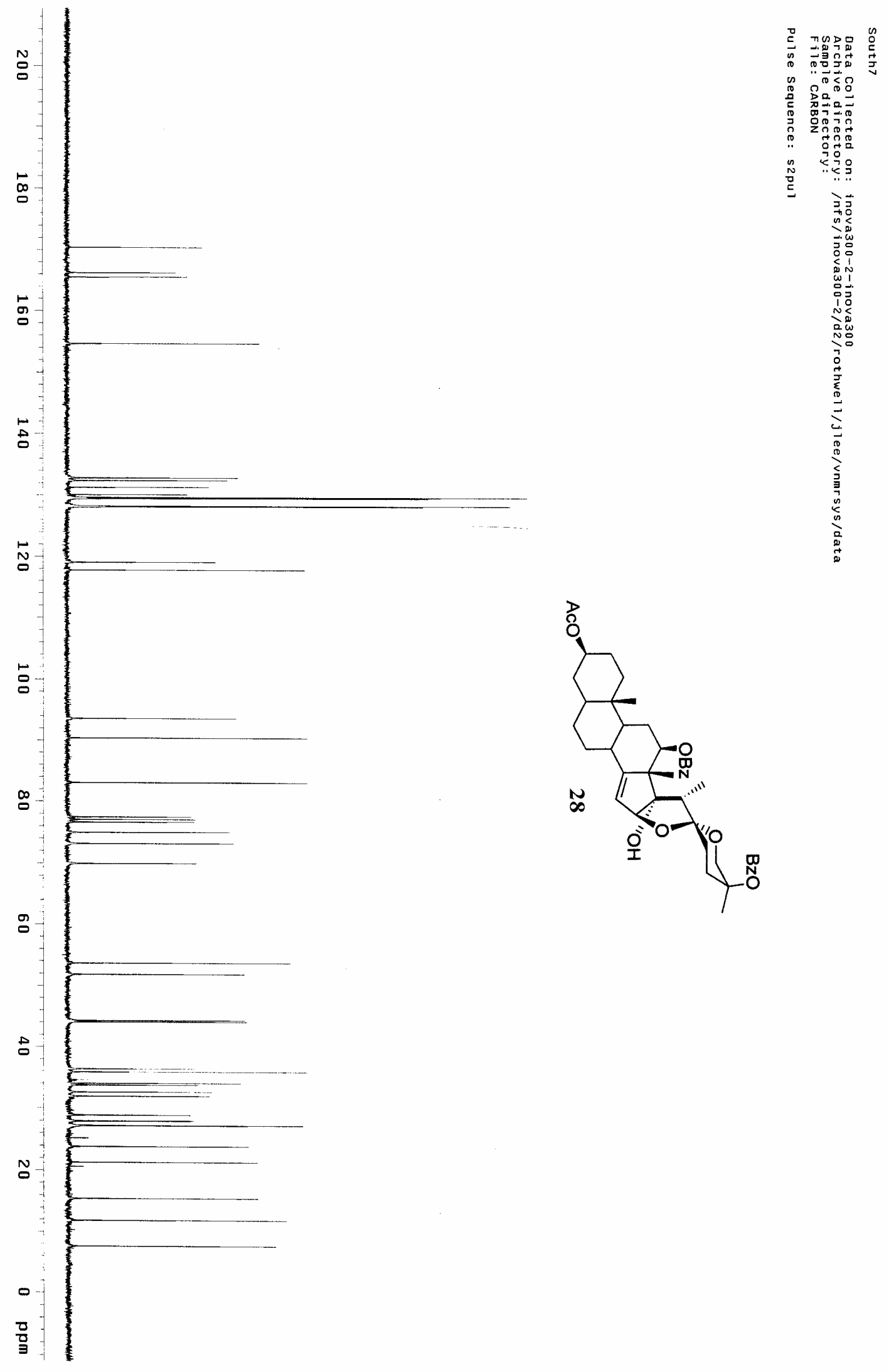




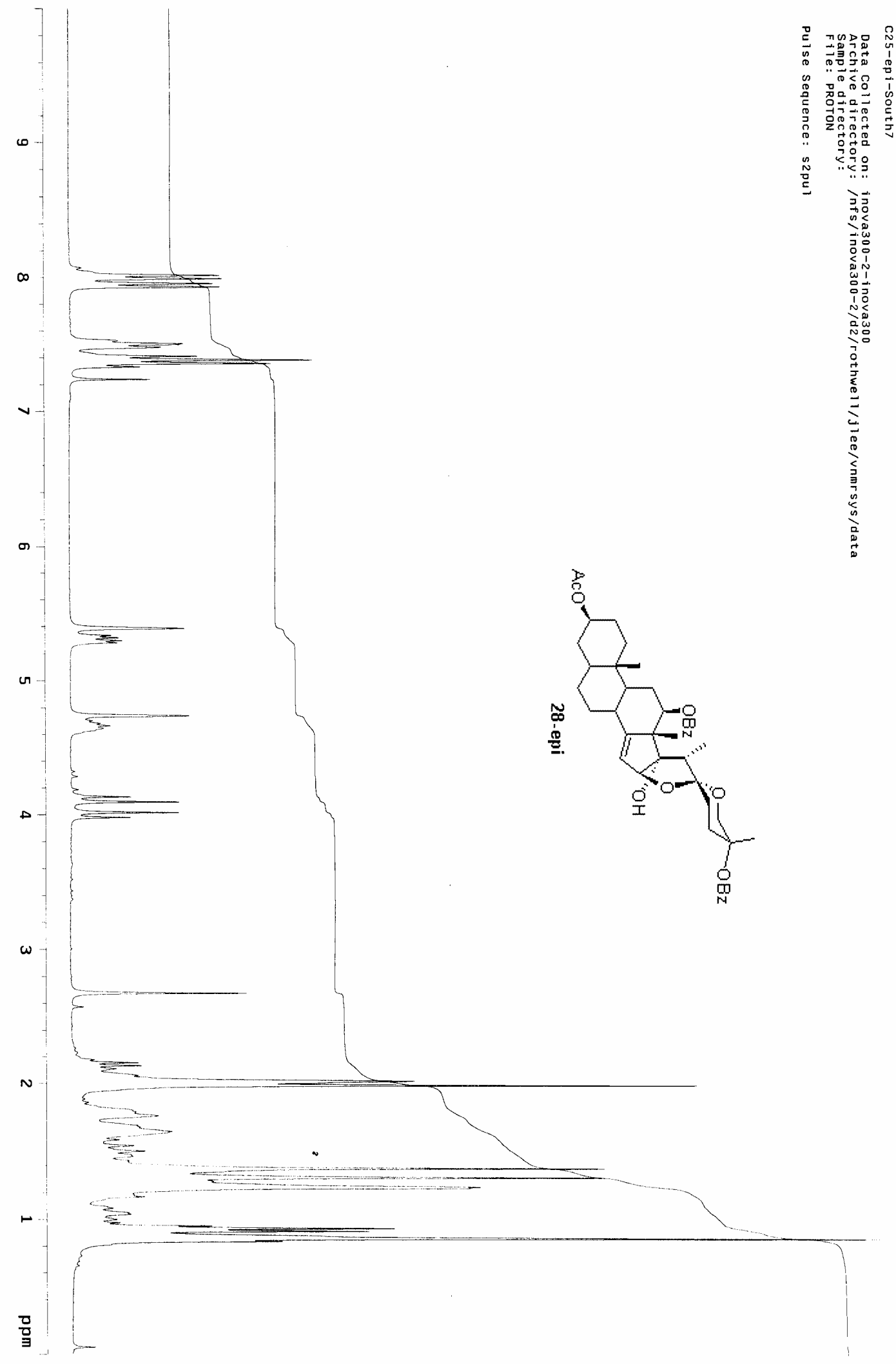

S- 78 

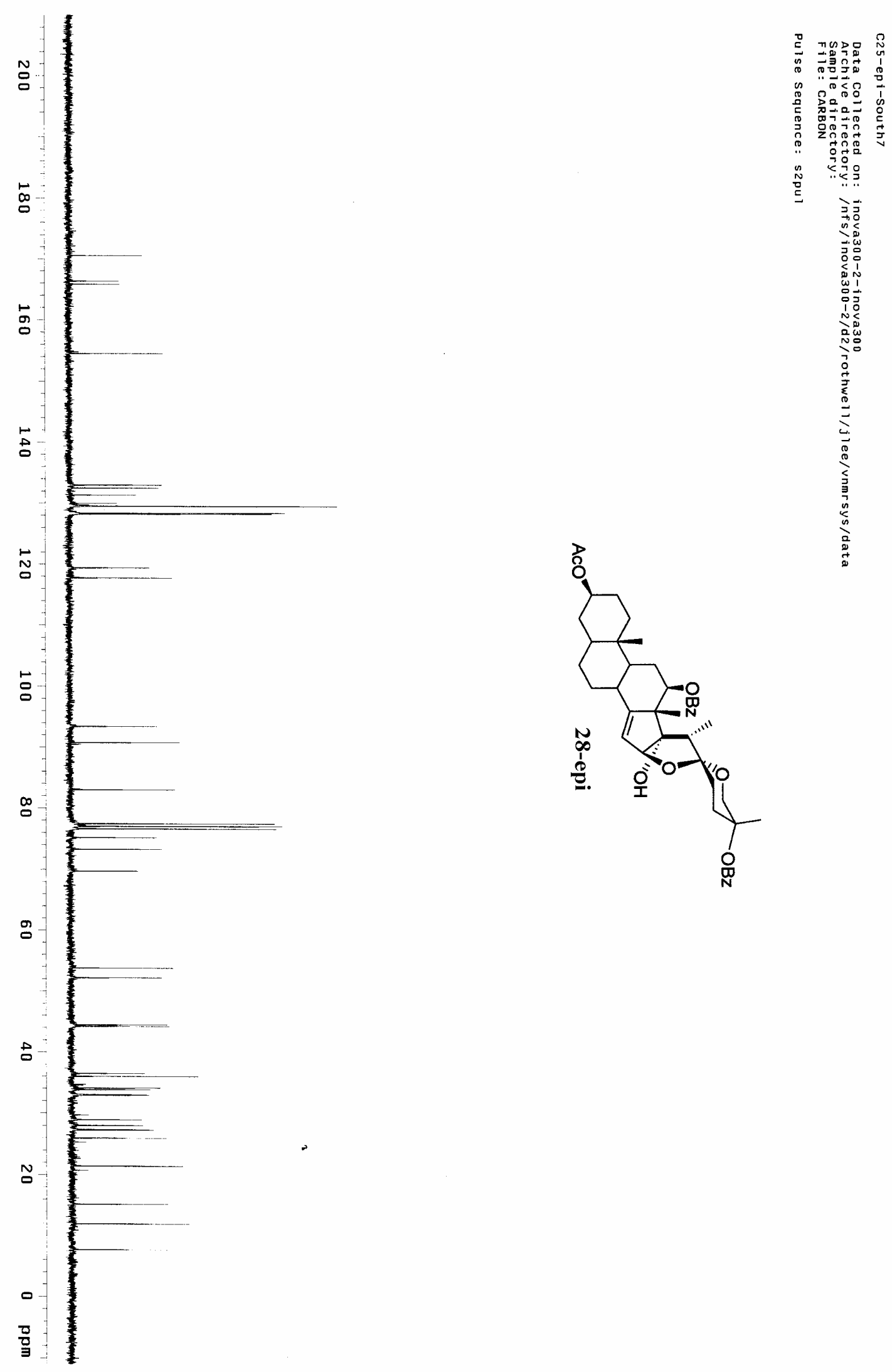


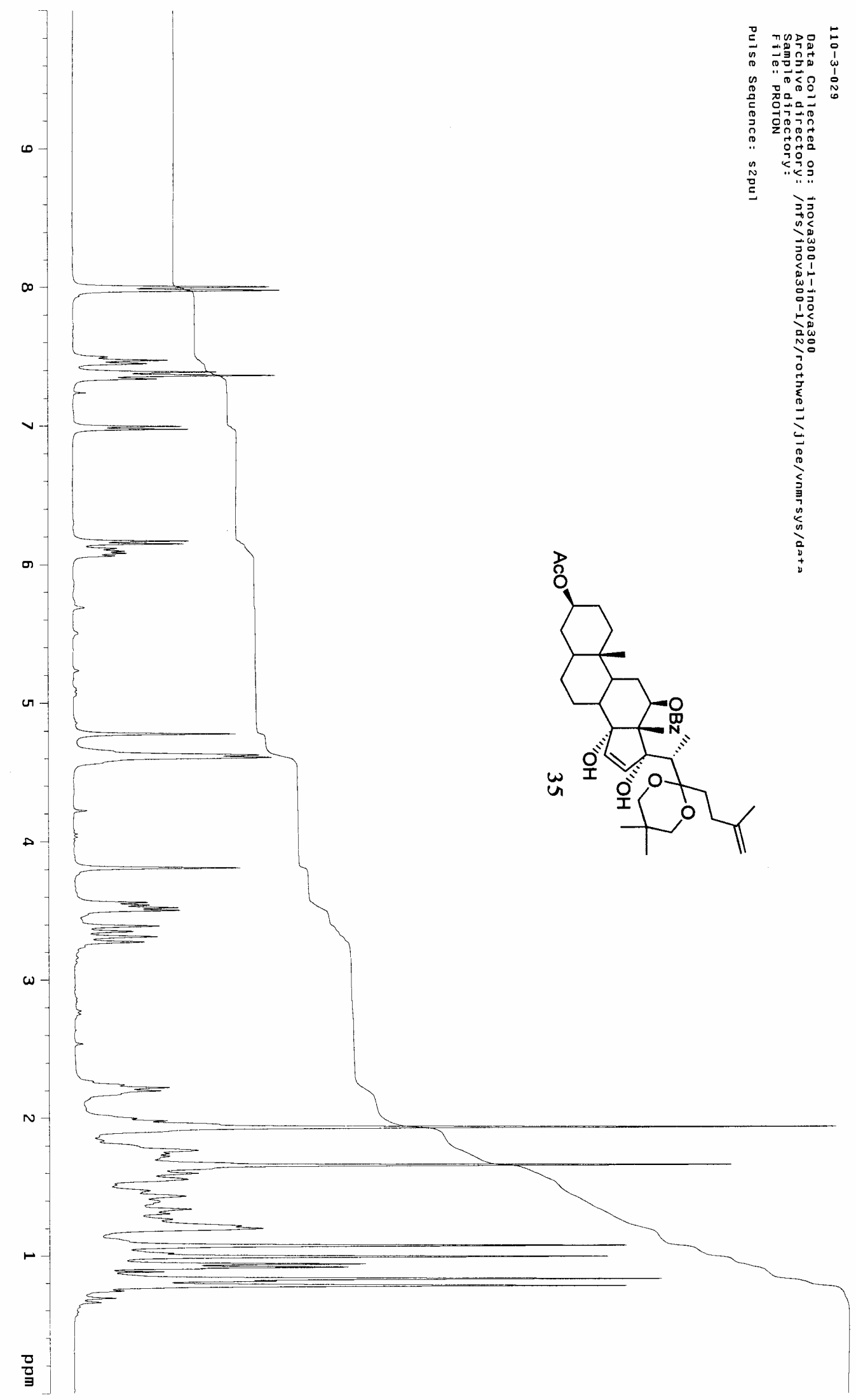

S- 80 

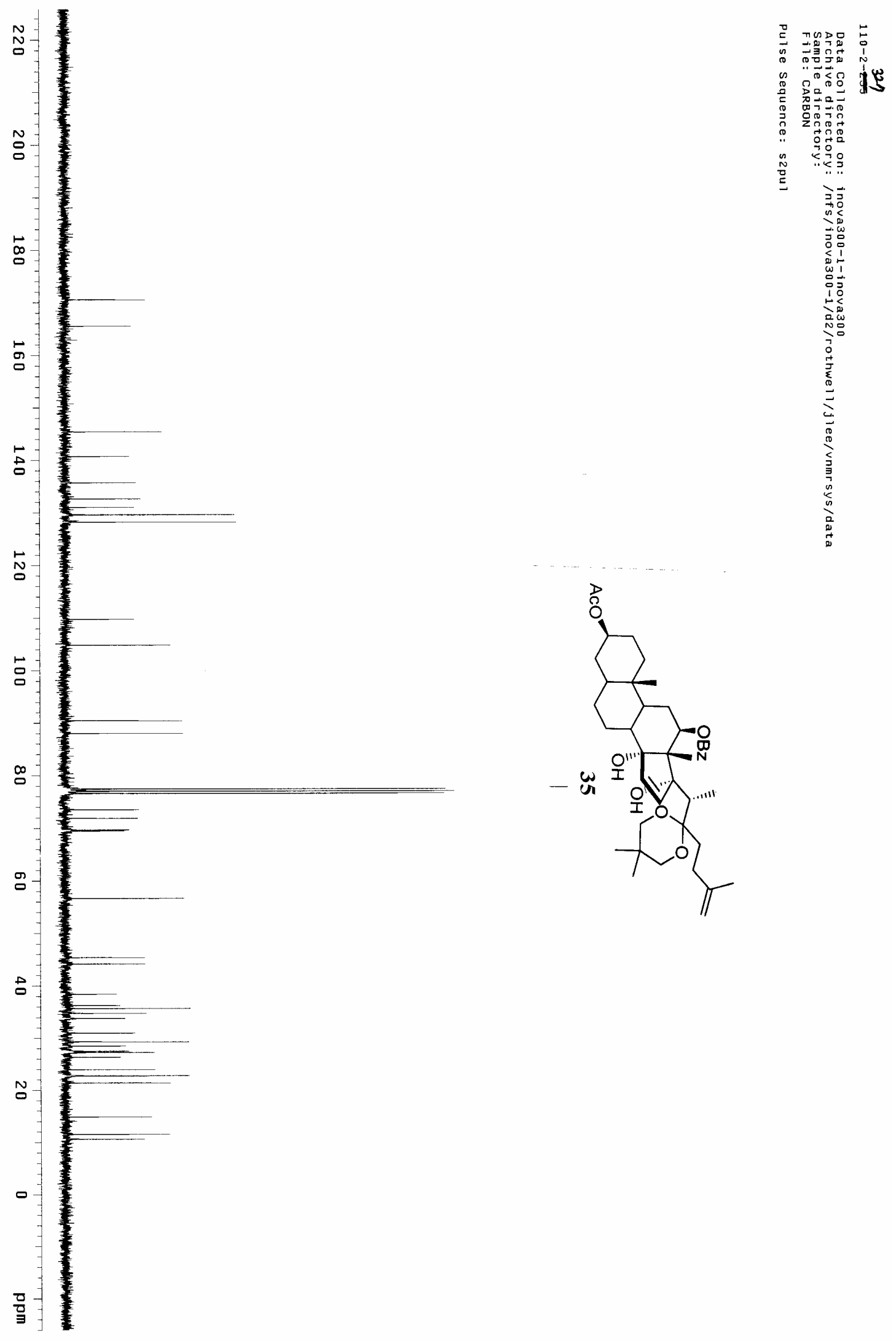

S- 81 


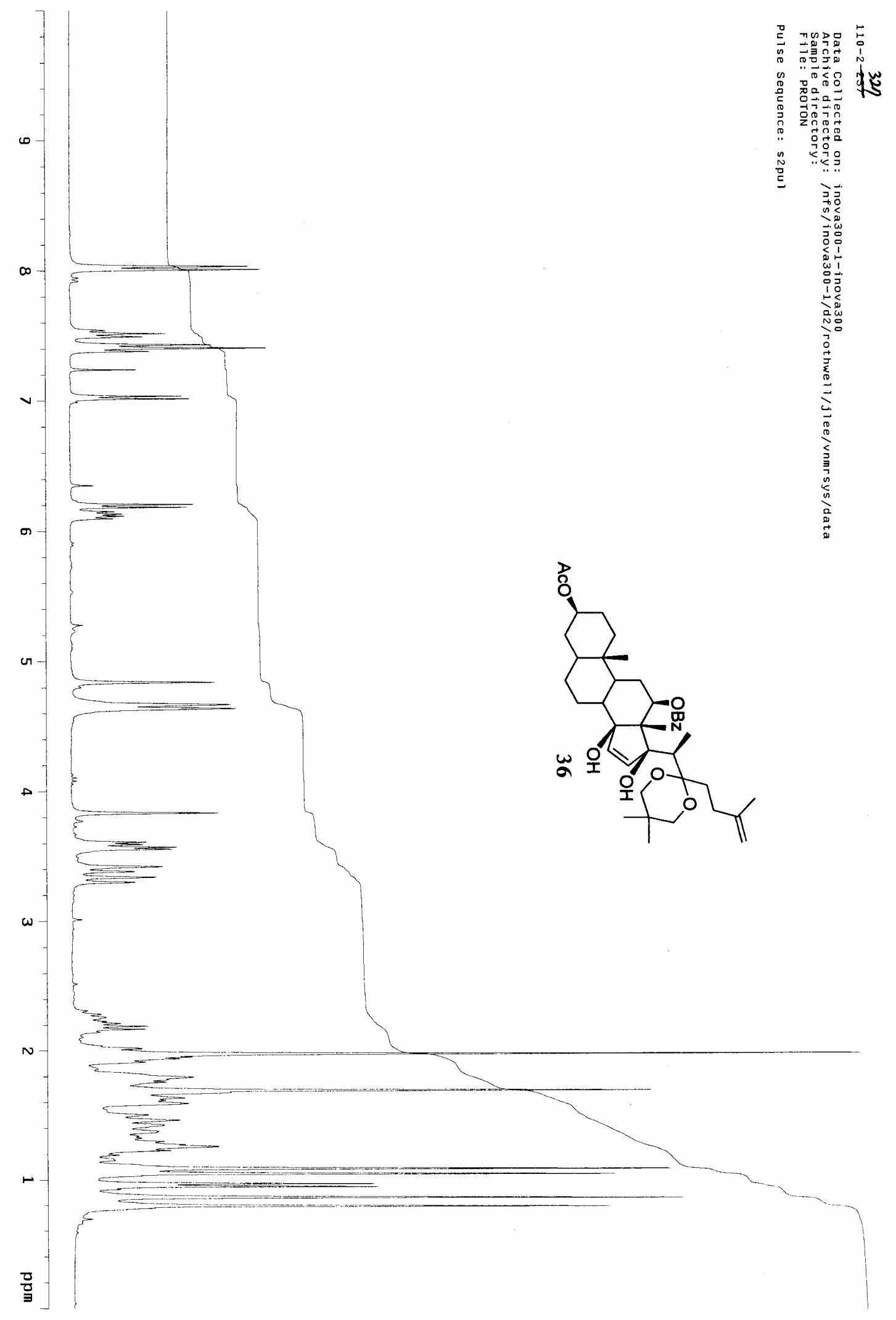

S- 82 

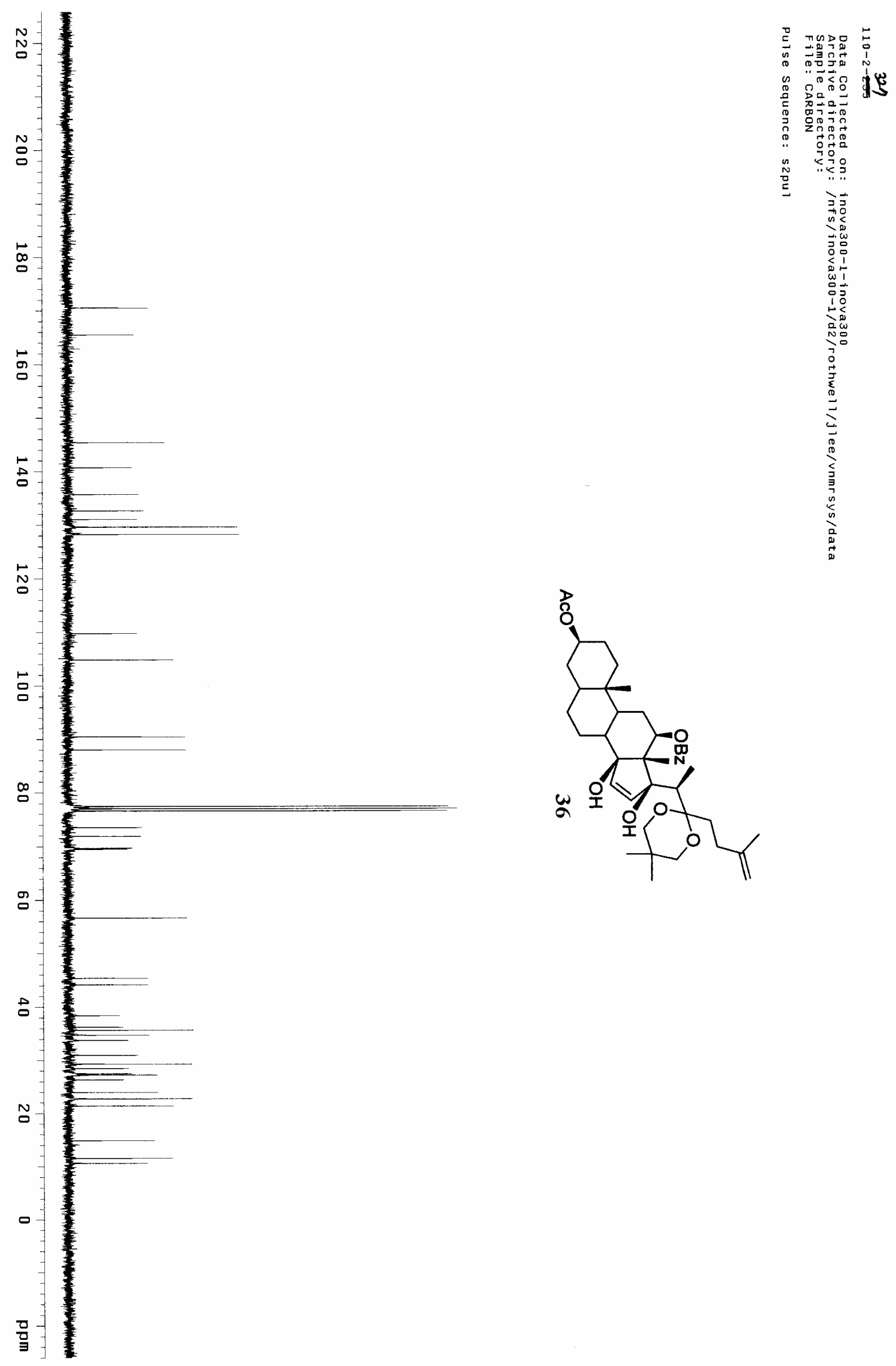


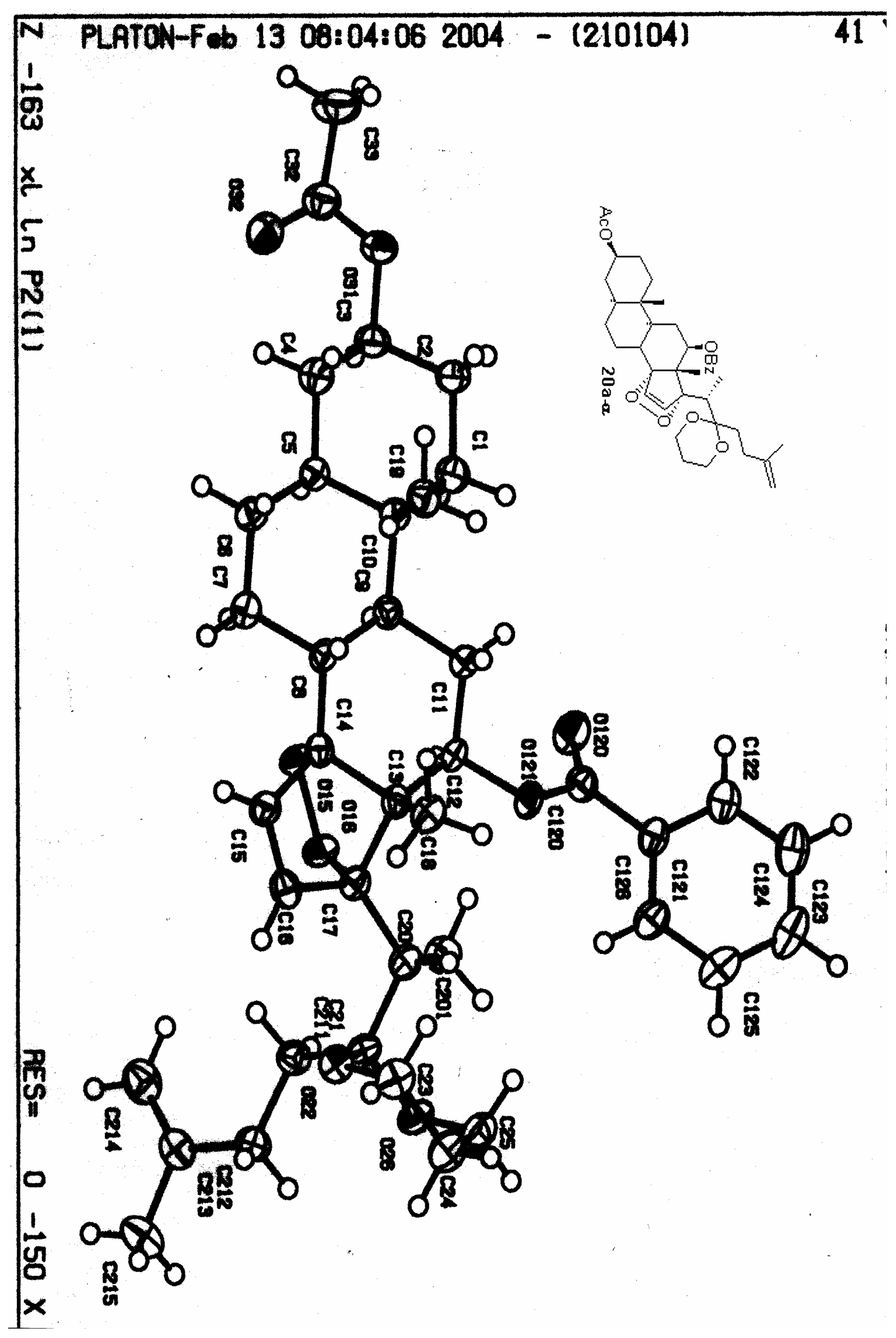

S- 84 